\title{
11. LOWER EOCENE CALCAREOUS NANNOFOSSIL BIOSTRATIGRAPHY BENEATH THE ATLANTIC SLOPE AND UPPER RISE OFF NEW JERSEY - NEW ZONATION BASED ON DEEP SEA DRILLING PROJECT SITES 612 AND 6131
}

\author{
Page C. Valentine, U.S. Geological Survey, Woods Hole, Massachusetts ${ }^{2}$
}

\begin{abstract}
Lower Eocene calcareous nannofossil limestone cored at DSDP Site 612 on the middle slope off New Jersey represents an almost complete biostratigraphic sequence; only the lowest biozone (CP9a; NP10*) was not recovered. The thickness of the strata $(198 \mathrm{~m})$, the good preservation of the nannofossils, and the lack of long hiatuses justify the acceptance of this section as a lower Eocene reference for the western North Atlantic margin. The widely recognized and very similar nannofossil zonations of Martini (NP zones) and Bukry-Okada (CP zones) are emended slightly to make their lower Eocene biozones coeval; in addition, five new subzones are erected that subdivide zones CP10 and CP11 (NP12 and NP13). Established biozone names are retained as they are altered little in concept, but alphanumeric code systems are changed somewhat by appending an asterisk (*) to identify zones that are emended. Zone CP10* (NP12*) is divided into two parts, the Lophodolithus nascens Subzone (CP10*a; NP12*a) and the Helicosphaera seminulum Subzone $\left(\mathrm{CP} 10^{*} \mathrm{~b}\right.$; NP12*b). Zone CP11* $\left(\mathrm{NP}^{*} 3^{*}\right)$ is divided into three parts, the Helicosphaera lophota Subzone (CP11*a; NP13*a), the Cyclicargolithus pseudogammation Subzone (CP11*b; NP13*b), and the Rhabdosphaera tenuis Subzone $\left(C P 1{ }^{*} \mathrm{c} ; \mathrm{NP13}{ }^{*} \mathrm{c}\right)$. At Site 612 , a time-depth curve based on nannofossil datums dated in previous studies reveals a smoothly declining sediment accumulation rate, from $4.9 \mathrm{~cm} / 10^{3} \mathrm{yr}$. in CP10* (NP12*) to $2.8 \mathrm{~cm} / 10^{3} \mathrm{yr}$. in CP12* (NP14*). The ages of first-occurrence datums not previously dated are approximated by projection onto this timedepth curve and are as follows: Helicosphaera seminulum, 55.0 Ma; Helicosphaera lophota, $54.5 \mathrm{Ma}$; Cyclicargolithus pseudogammation, $53.7 \mathrm{Ma}$; Rhabdosphaera tenuis, $52.6 \mathrm{Ma}$; and Rhabdosphaera inflata, 50.2 Ma. At nearby Site 613 on the upper rise, strata of similar age, $139 \mathrm{~m}$ thick, contain an unconformity representing Subzone CP11*b (NP13*b) and a hiatus of approximately $1.1 \mathrm{~m} . \mathrm{y}$. duration. The sediment accumulation rate in the lower part of this section ( 9.7 $\mathrm{cm} / 10^{3} \mathrm{yr}$.) is twice that observed for equivalent strata at Site 612. The hiatus and the heightened sediment accumulation rate at Site 613 probably represent the effects of episodic mass wasting on the early Eocene continental slope and rise.
\end{abstract}

\section{INTRODUCTION}

During Deep Sea Drilling Project Leg 95, core holes at Sites 612 and 613 on the continental margin off New Jersey penetrated a thick section of richly fossiliferous Eocene calcareous nannofossil limestone (Fig. 1). Eocene strata are widespread beneath the U.S. Atlantic margin. Beneath the outer shelf and upper slope the section is 400 to $500 \mathrm{~m}$ thick, but it thins both to seaward beneath the rise and to landward beneath the coastal plain, where erosional unconformities are evident.

The sections at Sites 612 and 613 represent an almost complete sequence of lower Eocene nannofossil biozones. In all, smear slides from 101 samples were examined in a 221-m section from Site 612 on the middle slope (water depth $1404 \mathrm{~m}$ ) and from 55 samples in a 148-m section from Site 613 on the uppermost rise (water depth 2323 $\mathrm{m})$. No long hiatuses are present in the lower Eocene section at Site 612. However, one subzone present at Site 612 is missing at Site 613. At neither site was the lowest subzone of the lower Eocene (CP9a; NP10*) recovered. In this study, emphasis is placed on nannofossil species that are present consistently throughout their ranges, that are relatively easy to identify in transmitted light, and that appear to be least affected by dissolution. Species

\footnotetext{
${ }^{1}$ Poag, C. W., Watts, A. B., et al., Init. Repts, DSDP, 95: Washington (U.S. Govt. Printing Office).

2 Address: U.S. Geological Survey, Woods Hole, MA 02543
}

that do not fit these criteria were noted for future study but are not treated here.

The completeness of the lower Eocene sections at Site 612 and 613 , and the fact that the ranges of many species could be determined reliably, presented an opportunity not only to revise and refine established lower Eocene nannofossil zonations of Martini $(1970,1971)$, Bukry $(1973,1975)$, and Okada and Bukry $(1980)$, but also to estimate the ages of first-occurrence datums (FADs) of stratigraphically important species, and to evaluate the sediment accumulation rates at the middle slope and upper rise sites. I regard the lower Eocene penetrated at Site 612 as a reference section for the western North Atlantic margin. This study is the initial step in an effort to reexamine Eocene biostratigraphy of the offshore Atlantic margin on the basis of drilling results at DSDP Site 612 and 613.

The revision of biozones in this study, and the associated alteration of NP and CP code names, requires the following clarification. An asterisk (*) superscript on an alphanumeric code name (e.g., NP12*, CP10*) indicates that the zone has been revised and that it is being used in the emended sense. A code without an asterisk (e.g., NP12, CP10) refers to the zone in its original sense. Original codes are used, for the most part, in the early discussion of various lower Eocene zonation schemes and in the review of species ranges that precedes the section describing the present revision of the lower Eocene zonation. Because the revisions of biozone boundaries are minor with regard to geologic time, the ranges of species 


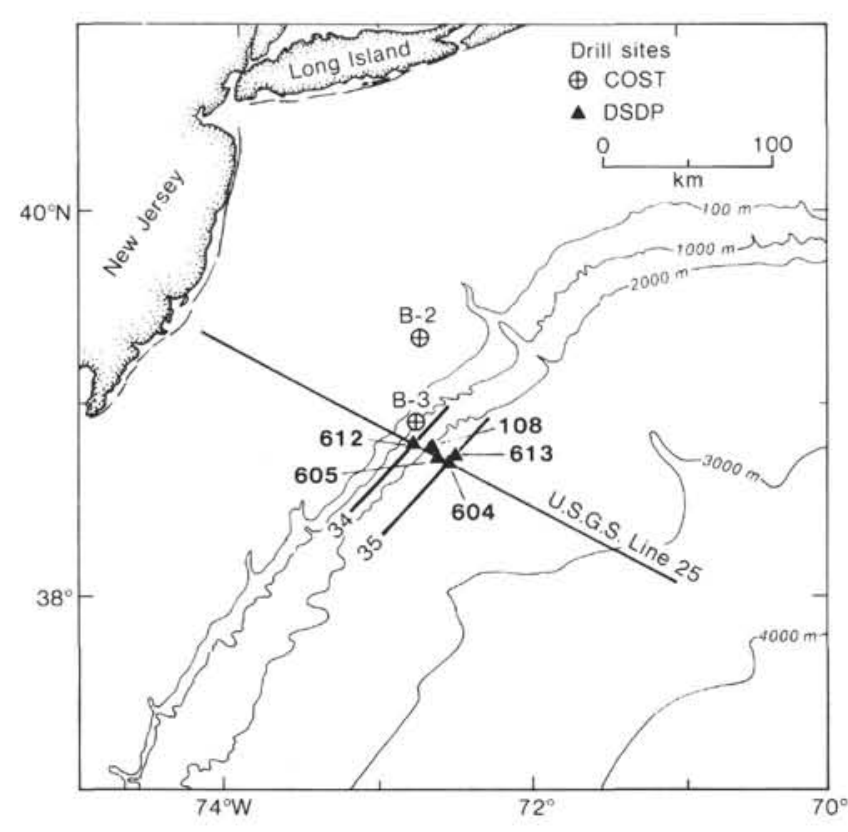

Figure 1. Map of the Atlantic margin off New Jersey, showing locations of Leg 95 Site 612 on the middle slope and Site 613 on the upper rise. Multichannel seismic lines 25, 34, and 35 cross these drill sites and Leg 93 Sites 604 and 605 and Leg 11 Site 108 on the upper rise. COST B-2 and B-3 are deep stratigraphic test wells drilled to depths of approximately $4900 \mathrm{~m}$ on the outer shelf and upper slope.

reported in the literature and discussed here in terms of unrevised NP and CP codes remain valid.

\section{PALEOGENE NANNOFOSSIL ZONATION}

The overall stratigraphy at Sites 612 and 613, and of the New Jersey margin as a whole, is treated elsewhere (e.g., Poag and Mountain, this volume). Eocene strata lying beneath the slope and upper rise are siliceous nannofossil limestone in which nannofossils generally are abundant and well preserved. Reworking is minimal and generally involves the rare occurrences of Upper Cretaceous forms. At Site 612 on the middle slope, the Eocene is approximately $415 \mathrm{~m}$ thick, and is remarkably complete except for an obvious hiatus between the middle and upper Eocene. At Site 613 on the upper rise, the Eocene section is only about $308 \mathrm{~m}$ thick because there is a major gap in the record between middle Eocene and Miocene strata. The lower Eocene at Site 612 is approximately $198 \mathrm{~m}$ thick, whereas it is about $139 \mathrm{~m}$ thick at Site 613. The difference of about $50 \mathrm{~m}$ in thickness of the lower Eocene sections at the two sites may be due in part to contemporaneous erosion by downslope mass wasting processes that apparently removed strata of Subzone CP11*b (NP13*b) at Site 613. Sediment composition is similar at the two sites, and there is no appreciable difference in the quartz content that would signal an increase in sediment transport from a continental source and thus account for the thicker section at the more landward Site 612 on the middle slope.

\section{Martini (NP) and Bukry-Okada (CP) Zonations}

The most widely applied nannofossil zonation schemes for Paleogene strata are the Martini $(1970,1971)$ NP zo- nation, based on the ranges of species chiefly from European sections and thus referred to as the standard or high-latitude zonation; and the Bukry-Okada CP zonation, based primarily on oceanic cored sections and referred to as the low-latitude or tropical zonation (Bukry, 1973, 1975, 1978, 1981; Okada and Bukry, 1980). The two schemes are in many ways comparable (Fig. 2). Although zonal names differ in some parts of the section, both zonations employ many of the same marker species for defining zonal boundaries. The Bukry-Okada zonation is somewhat more refined, incorporating 30 zones and subzones in the Paleogene to Martini's 25.

The Paleogene nannofossil zonation of Martini (1971) is based on biozones described chiefly by previous workers, in part emended by Martini. Although most of the reference sections for the 25 Martini zones are in Europe, including France (5), Germany (3), Switzerland (2), Denmark (2), England (1), Austria (1), and Belgium (1), many others are in other parts of the world, such as California (4), Trinidad (3), Cuba (2), and the USSR (1). The Bukry-Okada zones were described from the study of strata cored in the Atlantic, Pacific, and Indian ocean basins, except for several in the lower Paleocene based on strata in the North Atlantic basin. A recent monograph of Paleogene nannofossil biostratigraphy of northwestern Europe presented a useful summary of 22 Eocene zonations schemes erected by various authors between 1961 and 1980 for diverse regions of the world (Aubry, 1983). These authors subdivided the lower Eocene into four to five zones and subzones and, in general, relied on the same marker species for zonal boundaries. The present study refines this division by subdividing the Bukry-Okada CP10 and CP11 zones and the equivalent Martini NP12 and NP13 zones into five new subzones (Fig. 3).

The Martini and Bukry-Okada zonations for the lower Eocene and lowermost middle Eocene have many marker species in common (Fig. 2). Reference sections for the Martini zones are in Switzerland (NP10, NP11), Cuba (NP12, NP13), and California (NP14), whereas those for the Bukry-Okada zones (CP9-CP12) are in the major ocean basins. It appears that each of the lower Eocene biozones erected by these authors is comparable to a zone in the other scheme (Okada and Bukry, 1980; Bukry, 1981; Berggren, Kent, Flynn, and Van Couvering, 1985). The Martini zonation utilizes the following markers to bound five zones (NP10-NP14): Tribrachiatus nunnii, T. contortus, Discoaster lodoensis, T. orthostylus, D. sublodoensis, and Nannotetrina fulgens. Within the same stratigraphic interval, the Bukry-Okada scheme bounds four zones and four subzones with the first or last occurrences of Discoaster diastypus, Tribrachiatus contortus, D. lodoensis, Coccolithus crassus, $D$. sublodoensis, Nannotetrina fulgens, and Rhabdosphaera inflata. A major difference between the two zonations is that the Martini zonation uses the last occurrence of $T$. orthostylus as the datum to separate Zones NP12 and NP13, whereas the Bukry-Okada zonation uses the slightly older first occurrence of $C$. crassus to separate Zones CP10 and CP11, which encompass the same overall stratigraphic interval as NP12 and NP13 (Fig. 2). In the following discussion, for purposes of com- 


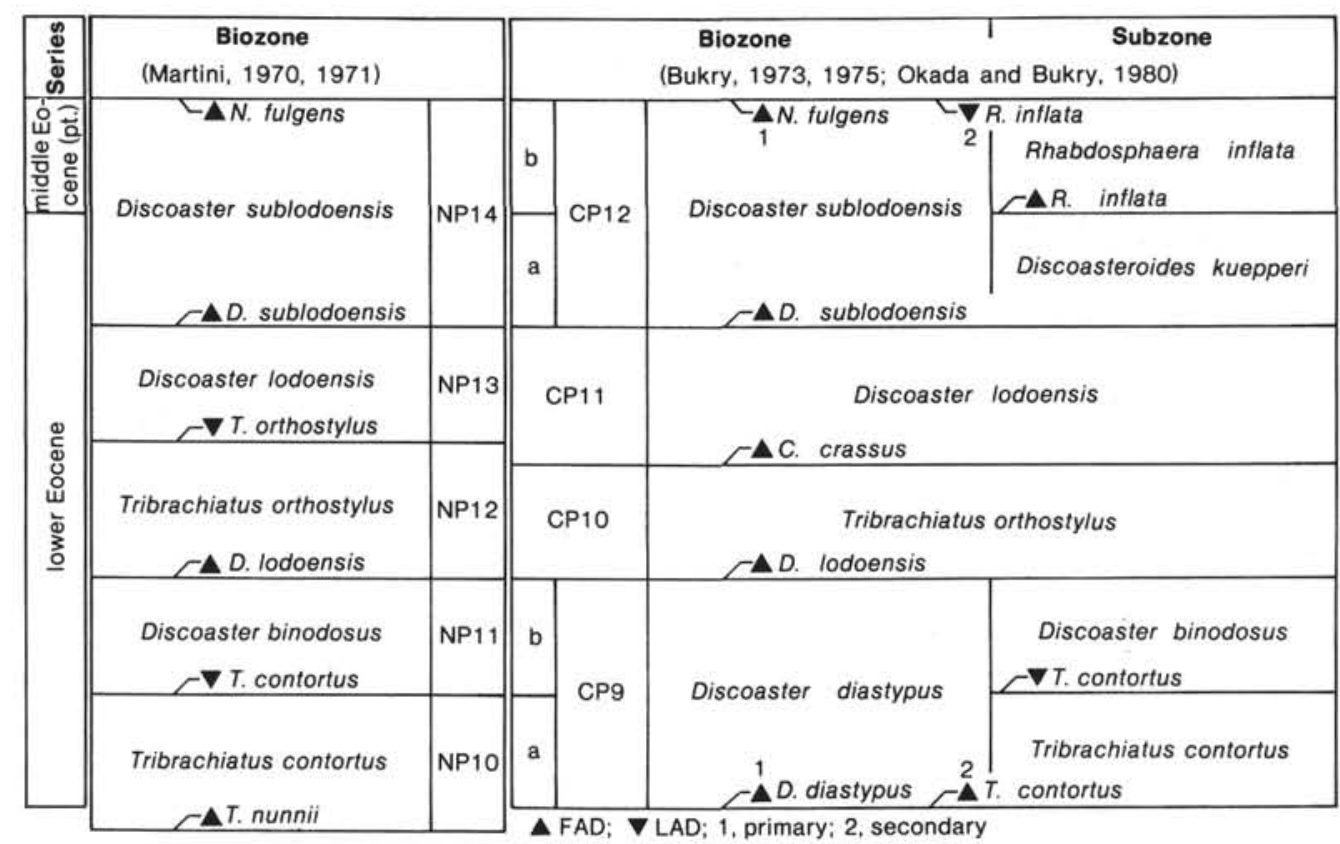

Figure 2. Comparison of Martini and Bukry-Okada lower Eocene nannofossil zonations. FAD, first-appearance datum; LAD, last-appearance datum. Note that zone boundaries are similar except for boundaries at base of Discoaster lodoensis Zone and at base of Tribrachiatus contortus Zone.

parison, the members of biozone pairs NP10 (CP9a), NP12 (CP10), and NP13 (CP11) are approximately equivalent in time; the other pairs NP11 (CP9b) and NP14 (CP12) are coeval by original definition of the authors.

The boundary marker species employed by both the Martini and Bukry-Okada zonations are present in the Anglo-Paris Basin, in a region where it has been shown that parts of only three lower Eocene zones, NP11, NP12, and NP13 (CP9b, CP10, and CP11), are present, because either the continental and shallow marine strata are unfossiliferous or there are unconformities in the section (Aubry, 1983, 1985). On the Atlantic margin off New Jersey, all the boundary markers of both the Martini and Bukry-Okada zonations are present in the lower Eocene at Sites 612 and 613 (the lowest part of the lower Eocene, Subzone CP9a, was not recovered). Furthermore, additional species chosen here to delineate new subzones by their first and last occurrences or to characterize zones by their appearance or disappearance within a zone, are also present in high- and low-latitude regions that include many localities both in the Anglo-Paris Basin (Aubry, 1983) and in the reference areas for the Martini and Bukry-Okada zonations (Martini, 1971; Bukry, 1973, 1975). These species include Chiasmolithus californicus, Cyclicargolithus pseudogammation, Ellipsolithus distichus, E. lajollaensis, E. macellus, Helicosphaera lophota, $H$. seminulum, Lophodolithus mochlophorus, L. nascens, the genus Reticulofenestra, and Rhabdosphaera tenuis. The offshore New Jersey strata can be correlated with either the Martini or Bukry-Okada biozones.

\section{RANGES OF STRATIGRAPHICALLY IMPORTANT SPECIES}

Within the lower Eocene section at Sites 612 and 613, the stratigraphic ranges of species such as Coccolithus crassus, Discoaster lodoensis, D. sublodoensis, Rhabdosphaera inflata, and Tribrachiatus orthostylus are consistent with ranges outlined in the Martini and BukryOkada zonations. During this study, it became evident that ranges could be determined for many species that heretofore had not been utilized as markers (Figs. 4, 5; Tables 1, 2). This is especially true for that part of the section that correlates with Zones CP10 and CP11 (NP12 and NP13).

Helicosphaera seminulum is not present in Subzone CP9b, the lowest Eocene recovered at Sites 612 and 613. It appears first in Sample 612-58,CC $(530.90 \mathrm{~m})$, some $17 \mathrm{~m}$ above the base of Zone CP10* (NP12*), and ranges upward through the lower Eocene section. The first occurrence of $H$. seminulum could be somewhat lower in the section because there was poor recovery in Core 612-58. However, it is not present in either Core 612-59, a full core also assigned to zone CP10* (NP12*), or in the partial Core 612-60 of Subzone CP9b (NP11). $H$. seminulum displays a similar range at Site 613 , where it occurs first in Sample 613-46,CC (524.90 m), about $53 \mathrm{~m}$ above the bottom of the hole, which terminates after penetrating about $19 \mathrm{~m}$ of strata assigned to Subzone CP9b (NP11). In a number of previous studies from diverse locations, the first appearance of $H$. seminulum was recorded to be in Zone NP12 (CP10) or its equivalent in the Anglo-Paris Basin (Aubry, 1983), in the Tallahatta Formation of Alabama and Georgia (Bybell and Gibson, 1985), and in the Lodo Formation of California (Bramlette and Sullivan, 1961). Mohler and Hay (1967) also reported its presence in the Lodo and "Tejon" formations of California, citing the work of Sullivan (1965). $H$. seminulum was not mentioned in the zonal descriptions of Martini (1971). However, Bukry (1973) first mentioned it in his zonal descriptions, based on oceanic samples, as part of the assemblage of Zone CP10; and he 


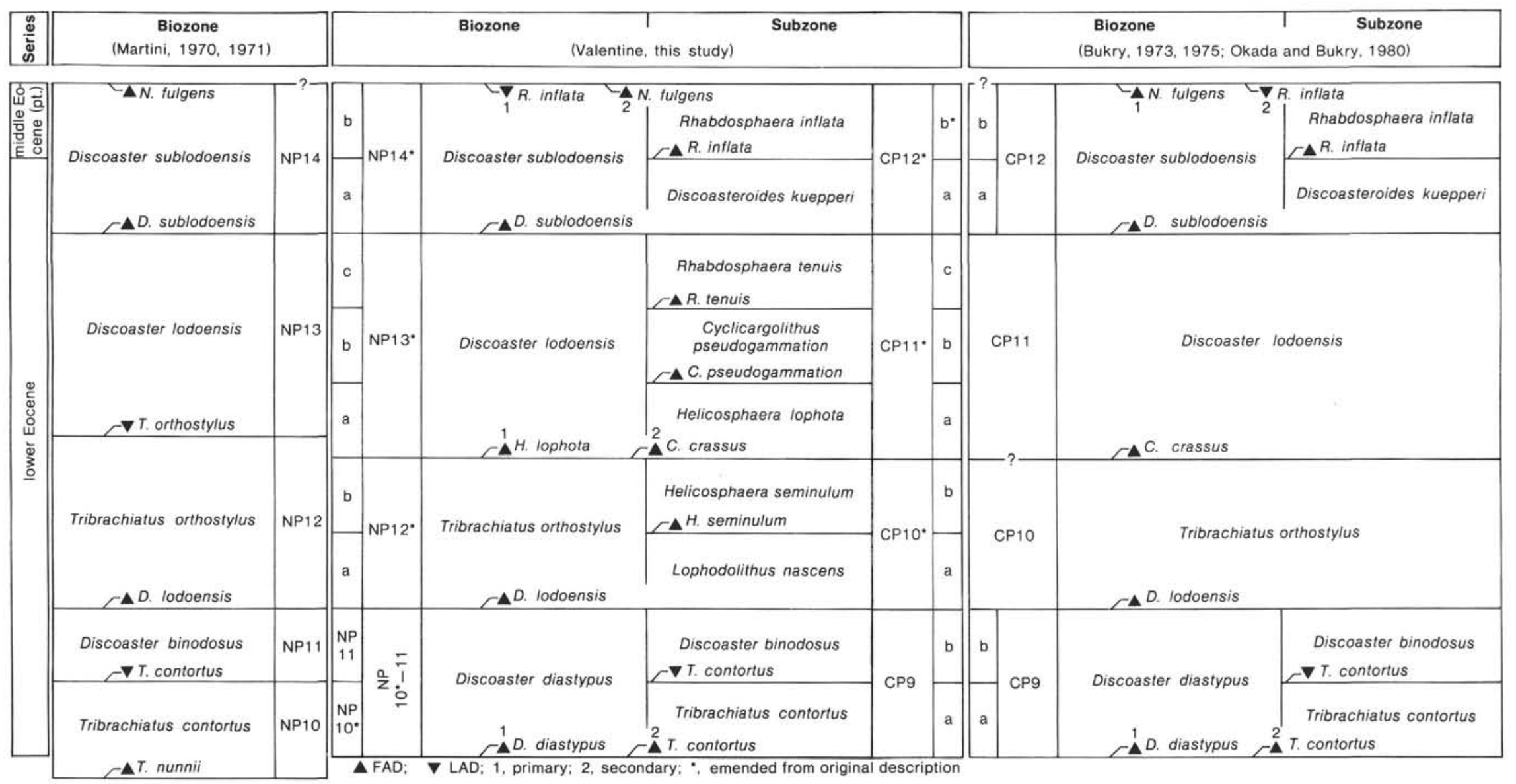

Figure 3. Comparison of the Martini and Bukry-Okada lower Eocene nannofossil zonations and the emended zonation of this study. FAD, first-appearance datum; LAD, last-appearance datum. The new zonation includes five new subzones (CP10*a,b, CP11*a,b,c; NP12*a,b, NP13*a,b,c), a revision of the old CP10/CP11 and NP12/NP13 zonal boundaries, and a preference for the use of the LAD of Rhabdosphaera inflata rather than the FAD of Nannotetrina fulgens as the primary datum for the top of Subzone CP12*b* (NP14*b). The widely accepted alphanumeric code systems that are part of the Martini and Bukry-Okada zonations are retained, but an asterisk (*) superscript has been added to indicate a revision of the original description. Datums that may not be coeval with those of this study are queried. 


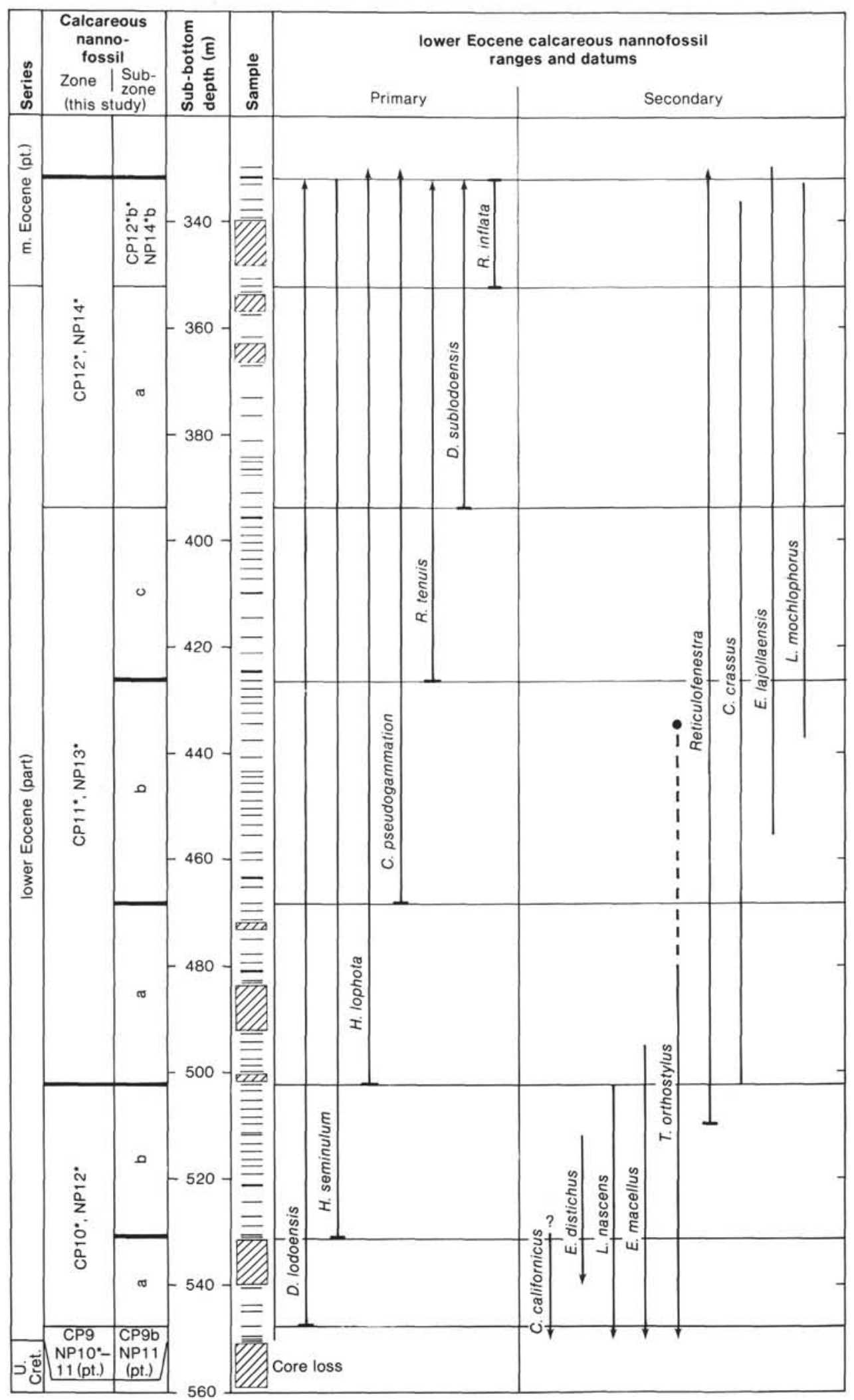

Figure 4. Ranges and datums of primary and secondary nannofossil markers in the lower Eocene at Site 612 , New Jersey slope. Datums of primary markers define zonal and subzonal boundaries. Secondary markers are important constituents of the assemblage, although their range terminations may not be precisely known. Lines terminated by arrows indicate that species is known to range into rocks older or younger than those represented here; lines with no termination indicate species range not yet determined; dot represents reworked $T$. orthostylus specimen. Bold (sub)zonal boundaries are newly defined or revised in this study. Asterisk (*) superscripts on CP and NP codes indicate revision of original zone or subzone. 


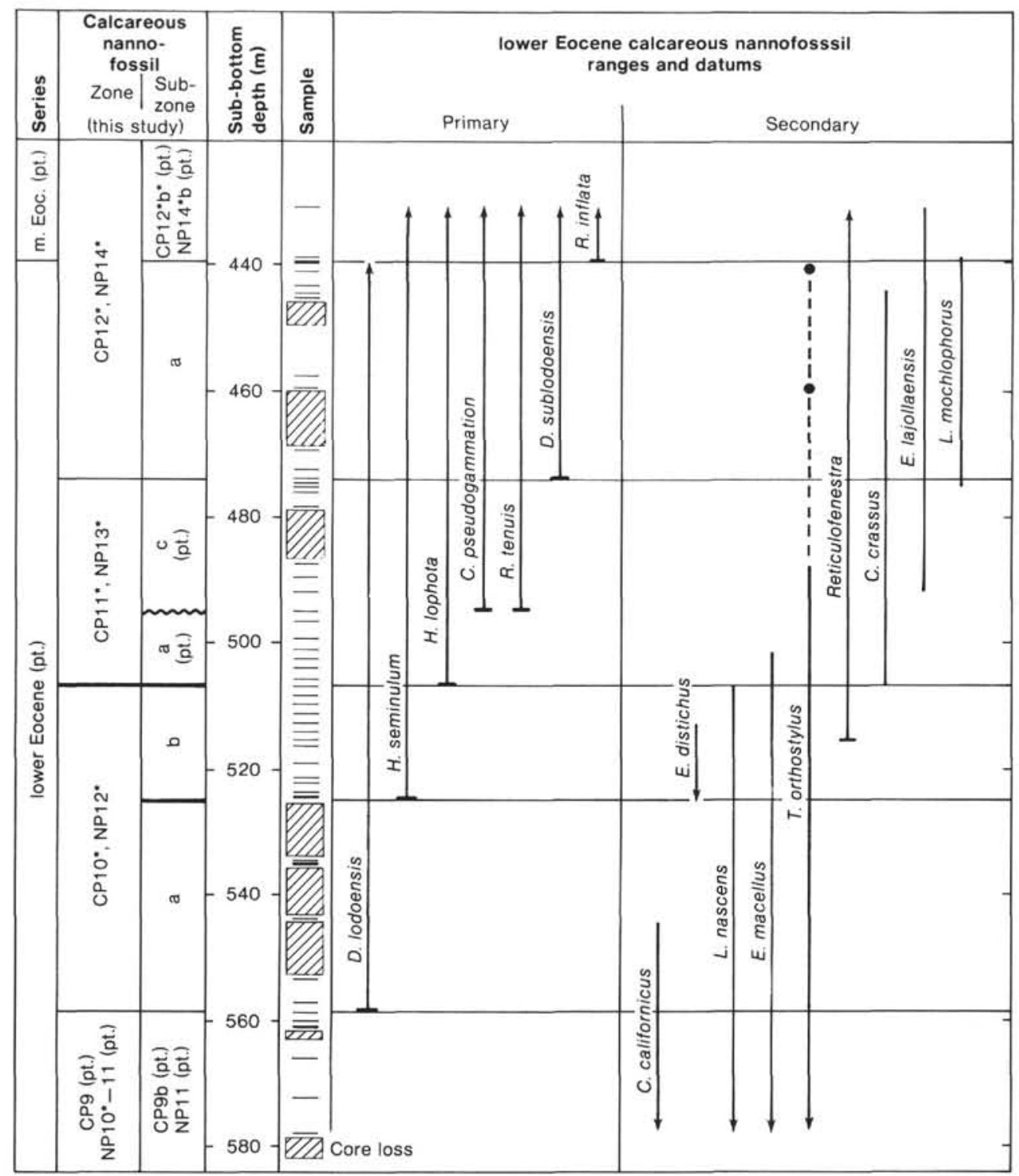

Figure 5. Ranges and datums of primary and secondary nannofossil markers in the lower Eocene at Site 613, New Jersey rise. See Figure 4 caption. This section is not as thick as that at Site 612. An unconformity is present at approximately $495 \mathrm{~m}$, and Subzone CP11*b (NP13*b) is not represented.

observed it in a sample (Mf2049) from the Lucia Mudstone in California that was also assigned to Zone CP10 (Bukry et al., 1977). Romein (1979) reported the first occurrence of $H$. seminulum in the $T$. orthostylus Zone (NP12; CP10) of the Caravaca and Aspe sections in southeastern Spain and in the Nahal Avdat section in Israel. The first occurrence of $H$. seminulum is the datum separating the newly defined Subzones CP10*a and CP10*b (NP12*a and NP12*b).

Chiasmolithus californicus is present in Subzone CP9b (NP11) at Site 612, and ranges into Zone CP10* (NP12*) to Sample $612-57 \mathrm{CC}(530.40 \mathrm{~m})$, just above the first occurrence of $H$. seminulum, where it ceases to be a persistent member of the flora. It is absent for several tens of meters above this level, but may occur sporadically up into Zone CP11* (NP13*), although identification is difficult. At Site 613, C. californicus also ranges into Zone CP10*, but it apparently becomes sporadic well below the first occurrence of $H$. seminulum. C. californicus occurs on a limited basis in Paleocene and lower Eocene strata in northwestern Europe (Aubry, 1983). Mohler and Hay (1967) and Martini (1971) did not mention it in their zonal descriptions. Bukry (1973) listed it as a member of assemblages characteristic of Paleocene Zones CP4 to CP7, but not in the lower Eocene. Bramlette and Sullivan (1961) reported it (Coccolithus aff. C. gigas) in the equivalent of Zones CP6 and CP7 and, sporadically, in CP11 from the Lodo Formation (California), but not in CP12. Gartner (1970) indicated that it ranged from the upper Paleocene to the lower Eocene, but that its occurrences in the upper part of its range were sporadic and that it sometimes was difficult to differentiate from the related species, $C$. consuetus. Gartner (1971) reported its occurrence on the Blake Plateau in the equivalent of Zones CP10 and CP11 and even higher in the section. The last occurrence of $C$. califor- 
nicus has not proved to be a reliable datum. The results of the present study suggest that it last occurs with regularity in assemblages from Zone CP10* (NP12*).

Helicosphaera lophota first appears in Sample 612$55-1,20-22 \mathrm{~cm}(502.21 \mathrm{~m})$, at the top of Zone CP10* (NP12*), higher in the section than $H$. seminulum, and it ranges through the lower Eocene. At Site 613, H. lopho$t a$ first appears in Sample 613-45-1, 90-91 cm (506.81 m), likewise higher than $H$. seminulum. Aubry (1983) showed $H$. lophota first occurring in Zone NP15 of the middle Eocene in the Anglo-Paris Basin, whereas Martini (1971) indicated a possible first occurrence in NP13 (CP11), and Bukry (1973) listed H. sp. cf. H. lophota in CP11. Mohler and Hay (1967) reported its presence in the equivalent of Zone NP12 (CP10) on the basis of Sullivan's (1965) study of the Lodo and "Tejon" formations (California). Bukry et al. (1977) observed H. lophota in a sample (Mf2050) from the Lucia Mudstone (California), assigned to Zone CP10 but most likely Zone CP11 (NP13), on the basis of the reported presence of Cyclicargolithus pseudogammation, Reticulofenestra dictyoda, and Rhabdosphaera tenuis, and the absence of Tribachiatus orthostylus. Bybell and Gibson (1985) placed the first occurrence of $H$. lophota high in Zone NP12 (CP10) in the Tallahatta Formation of Alabama and Georgia and just below the last occurrence of T. orthostylus. Several studies of the Lodo Formation in Lodo Gulch, California (Bramlette and Sullivan, 1961; Poore, 1976; Warren, 1983) showed that the first occurrence of $H$. lophota is above that of $H$. seminulum and practically coincides with the first occurrence of Coccolithus crassus, the datum marking the top of the T. orthostylus Zone (CP10) of Bukry (1973). In the same section, H. lopho$t a$ first appears below the last occurrence of $T$. orthostylus, the datum marking the top of the $T$. orthostylus Zone (NP12) of Brönnimann and Stradner (1960). The reported first occurrences of $H$. lophota and the results of the present study suggest that this datum is between the first occurrence of $H$. seminulum (below) and the last occurrence of $T$. orthostylus (above). The first occurrence of $H$. lophota was reported however, to be slightly above (1-2 m) the last occurrence of $T$. orthostylus in the Aspe section of southeastern Spain and in the Nahal Avdat section in Israel (Romein, 1979), and further study of these two sections is required. The first occurrence of $H$. lophota is the newly revised datum separating Zones CP10* and CP11* (NP12* and NP13*).

Lophodolithus nascens is present in almost all samples studied from Subzone CP9b (NP11) and Zone CP10* (NP12*) at Site 612, and ranges up to Sample 612-55-1, $20-22 \mathrm{~cm}(502.21 \mathrm{~m})$; its last occurrence coincides with the first occurrence of $\mathrm{H}$. lophota at the base of CP11* (NP13*). The same relationship exists at Site 613, where $L$. nascens last occurs in Sample 613-45-1, 90-91 cm $(506.81 \mathrm{~m})$. At Site 612 a closely related species, L. mochlophorus, first appears higher in Zone CP11* (NP13*) in Sample 612-48-3, 20-22 cm (437.51 m), and it ranges into the middle Eocene. The ranges of $L$. nascens and L. mochlophorus are separated by a similar gap at Site 613. By contrast, in several previous studies, the ranges of $L$. nascens and $L$. mochlophorus were reported to be consecutive or to overlap slightly (Bramlette and Sullivan, 1961; Mohler and Hay, 1967; Bukry, 1973). L. nascens was reported to last occur in the upper part of Zone NP14 (CP12b) in the Anglo-Paris Basin (Aubry, 1983) and in the Tallahatta Formation of Alabama and Georgia (Bybell and Gibson, 1985), and in the equivalent of CP12a in the Lodo Formation, California (Bramlette and Sullivan, 1961). Mohler and Hay (1967), Hay (1967), and Bukry (1973) included $L$. nascens in the assemblage of Zone NP13 (CP11), but not in the lower part of Zone NP14 (CP12a), where L. mochlophorus was first listed. Bybell and Gibson (1985) also reported the first occurrence of L. mochlophorus in the lower part of NP14 (CP12a). L. mochlophorus was reported to first occur in the equivalent of Subzone CP12a in biostratigraphic Unit 4 (Domengine Formation, California) of Bramlette and Sullivan (1961), but Bukry et al. (1977) listed this species in an assemblage from an unnamed shale (Mf2051) in California that was assigned to Zone CP11. Bramlette and Sullivan (1961) stated that $L$. mochlophorus probably developed from $L$. nascens. At Site 612, typical specimens of $L$. nascens, as illustrated by Bramlette and Sullivan (1961, plate 4) occur consistently up to $502.21 \mathrm{~m}$, where they disappear. Likewise, typical $L$. mochlophorus appear at $437.51 \mathrm{~m}$ at Site 612 and are present continuously up to $395.57 \mathrm{~m}$, where their presence becomes sporadic. Upon closer examination, it was found that specimens not readily assignable to either $L$. nascens or L. mochlophorus are present at Site 612 from about 440 to $460 \mathrm{~m}$. Further study is required to determine if these forms ( $L$. sp. cf. L. mochlophorus) are a separate species.

The genus Reticulofenestra at Site 612 first occurs in Sample 612-55-6, 20-22 cm (509.71 m) in Zone CP10* (NP12*), between the first occurrences of $H$. seminulum and $H$. lophota. The genus ranges up through the lower Eocene. At Site 613 it appears in Sample 613-46$1,20-21 \mathrm{~cm}(515.60 \mathrm{~m})$ and displays a similar range. The first appearances of species of Reticulofenestra that are considered stratigraphically important in previous studies include the following: $R$. coenura and $R$. dictyoda generally in NP14 (CP12) of the Anglo-Paris Basin, but $R$. dictyoda is present in NP12 (CP10) in the Bracklesham Beds on the Isle of Wight (Aubry, 1983); $R$. dictyoda possibly in NP13 (Martini, 1971); $R$. coenura and $R$. dictyoda in NP12 (CP10) of the Tallahatta Formation in Alabama and Georgia (Bybell and Gibson, 1985); $R$. sp. cf. $R$. dictyoda in CP10, $R$. dictyoda in CP11., and $R$. samodurovi in CP12b (Bukry, 1973); $R$. dictyoda between the first occurrences of $H$. seminulum and $H$. lophota in NP12 (CP10) of the Aspe section in southeastern Spain, and $R$. dictyoda coinciding with the first occurrence of $H$. lophota in NP13 (CP11) of the Nahal Avdat section in Israel (Romein, 1979); and $R$. dictyoda listed in the assemblage for a sample (Mf2050) from the Lucia Mudstone (California) assigned to Zone CP10 (Bukry et al, 1977) but probably better assigned to Zone CP11. The genus Reticulofenestra is easy to identify, but the earliest species represented at Sites 612 and 613 are difficult to differentiate and their individual ranges are, at present, of little stratigraphic use. It appears that $R$. 
Table 1. Ranges of selected nannofossil species, Site 612, New Jersey slope.

\begin{tabular}{|c|c|c|c|c|c|c|c|c|c|c|c|c|c|c|c|c|c|c|c|c|c|c|c|c|c|c|c|}
\hline Series & Biozone & $\begin{array}{l}\text { reous } \\
\text { fossil Subzone } \\
\text { this study) }\end{array}$ & $\begin{array}{l}\text { Core-Section, } \\
\text { interval }(\mathrm{cm})\end{array}$ & $\begin{array}{l}\text { Sub-bottom } \\
\text { depth } \\
\text { (m) }\end{array}$ & 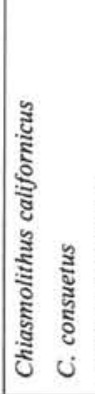 & 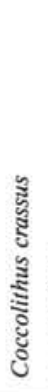 & & 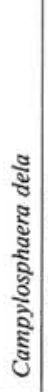 & 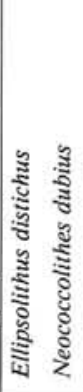 & 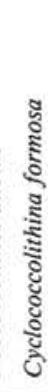 & 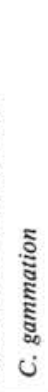 & 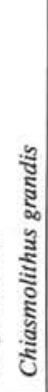 & 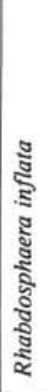 & 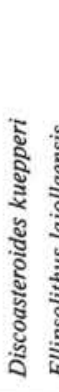 & 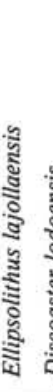 & 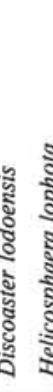 & & 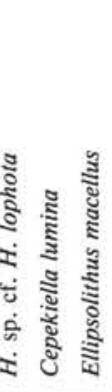 & 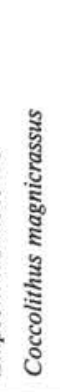 & 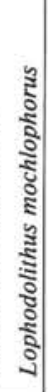 & 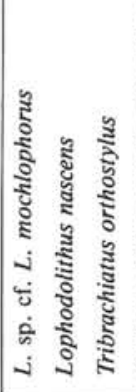 & 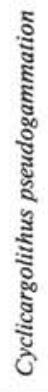 & 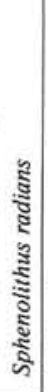 & 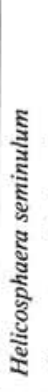 & 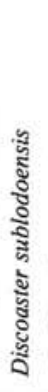 & 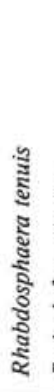 & 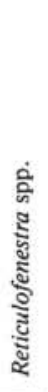 \\
\hline \multirow{5}{*}{$\begin{array}{l}\text { lower } \\
\text { Eocene } \\
\text { (part) }\end{array}$} & & & $\begin{array}{l}37-2,20-22 \\
37-3,54\end{array}$ & $\begin{array}{l}329.81 \\
331.64\end{array}$ & $x$ & & $\mathrm{x}$ & $x$ & $\begin{array}{l}\mathrm{x} \\
\mathrm{x}\end{array}$ & & & $\begin{array}{l}\mathrm{x} \\
\mathrm{x}\end{array}$ & & & $\begin{array}{ll}x & \\
x & x\end{array}$ & $x^{x}$ & & $\begin{array}{l}\mathrm{x} \\
\mathrm{x}\end{array}$ & & & & $\begin{array}{l}\mathrm{x} \\
\mathrm{x}\end{array}$ & $\mathrm{x}$ & $x$ & $x$ & $\mathrm{x}$ & $\begin{array}{l}\mathrm{x} \\
\mathrm{x}\end{array}$ \\
\hline & & $\begin{array}{l}\text { R. inflata Subzone } \\
\text { CP1 } 12^{*} b^{*}, \text { NP } 14^{*} \mathrm{~b}\end{array}$ & $\begin{array}{l}37-3,80 \\
37-4,20-22 \\
37-6,20-22 \\
37, \mathrm{CC} \\
38, \mathrm{CC} \\
39-3,20-22 \\
39-4,20-22\end{array}$ & $\begin{array}{l}331.90 \\
332.81 \\
335.81 \\
337.70 \\
339.34 \\
350.51 \\
352.01\end{array}$ & $\begin{array}{l}x \\
x \\
x \\
x \\
x\end{array}$ & $x$ & & $\begin{array}{l}x \\
x \\
x\end{array}$ & $\begin{array}{l}x \\
x \\
x \\
x \\
x \\
x\end{array}$ & $\begin{array}{l}\mathrm{x} \\
\mathrm{x} \\
\mathrm{x} \\
\mathrm{x} \\
\mathrm{x} \\
\mathrm{x} \\
\mathrm{x}\end{array}$ & $\begin{array}{l} \\
\mathrm{x} \\
\mathrm{x}\end{array}$ & \begin{tabular}{l|}
$\mathrm{x}$ \\
$\mathrm{x}$ \\
$\mathrm{x}$ \\
$\mathrm{x}$ \\
$\mathrm{x}$ \\
$\mathrm{x}$ \\
$\mathrm{x}$
\end{tabular} & $\begin{array}{l}x \\
x \\
x \\
x \\
x \\
x \\
x \\
\end{array}$ & & $\begin{array}{ll} & x \\
x & x \\
x & x \\
x & x \\
x & x \\
x & \\
\end{array}$ & $\begin{array}{ll}x & x \\
x & x \\
& x \\
x & \\
x & x \\
x & x \\
& x\end{array}$ & & $\begin{array}{l}x \\
x \\
x \\
x \\
x \\
x\end{array}$ & $\begin{array}{l}\mathrm{x} \\
\mathrm{x} \\
\mathrm{x} \\
\mathrm{x}\end{array}$ & $\begin{array}{l}x \\
x \\
x\end{array}$ & $x$ & $\begin{array}{l}\mathrm{x} \\
\mathrm{x} \\
\mathrm{x} \\
\mathrm{x} \\
\mathrm{x} \\
\mathrm{x} \\
\mathrm{x}\end{array}$ & $\begin{array}{l}x \\
x \\
x \\
x \\
x \\
x\end{array}$ & $\begin{array}{l}x \\
x \\
x \\
x \\
x \\
x \\
x\end{array}$ & $\begin{array}{l}x \\
x \\
x \\
x \\
x \\
x \\
x\end{array}$ & $\begin{array}{l}\mathrm{x} \\
\mathrm{x} \\
\mathrm{x} \\
\mathrm{x} \\
\mathrm{x} \\
\mathrm{x} \\
\mathrm{x} \\
\end{array}$ & $\begin{array}{l}x \\
x \\
x \\
x \\
x \\
x \\
x \\
\end{array}$ \\
\hline & $\begin{array}{l}\text { Discoaster sublodoensis } \\
\text { Zone CPI2*, NP14** }\end{array}$ & $\begin{array}{l}\text { D. kuepperi Subzone } \\
\text { CP12*a, NP14*a }\end{array}$ & $\begin{array}{l}39, \mathrm{CC} \\
40-1,20-22 \\
40-4,20-22 \\
41-1,20-22 \\
41-5,20-22 \\
41, \mathrm{CC} \\
42-4,20-22 \\
42-6,20-22 \\
42, \mathrm{CC} \\
43-1,20-22 \\
43-2,20-22 \\
43-4,20-22 \\
43-6,20-22\end{array}$ & $\begin{array}{l}353.40 \\
357.21 \\
361.71 \\
366.91 \\
372.91 \\
376.18 \\
381.11 \\
384.11 \\
384.88 \\
386.21 \\
387.71 \\
390.71 \\
393.71\end{array}$ & $\begin{array}{l}x \\
x \\
x \\
x\end{array}$ & $\begin{array}{l}\mathrm{x} \\
\mathrm{x} \\
\mathrm{x} \\
\mathrm{x} \\
\mathrm{x} \\
\mathrm{x} \\
\mathrm{x}\end{array}$ & $\begin{array}{l}x \\
x\end{array}$ & $\begin{array}{l}\mathrm{x} \\
\mathrm{x} \\
\mathrm{x} \\
\mathrm{x} \\
\mathrm{x} \\
\mathrm{x} \\
\mathrm{x} \\
\mathrm{x}\end{array}$ & $\begin{array}{l}\mathrm{x} \\
\mathrm{x} \\
\mathrm{x} \\
\mathrm{x} \\
\mathrm{x} \\
\mathrm{x} \\
\mathrm{x} \\
\mathrm{x} \\
\mathrm{x} \\
\mathrm{x} \\
\mathrm{x} \\
\mathrm{x} \\
\mathrm{x}\end{array}$ & $\begin{array}{l}\mathrm{x} \\
\mathrm{x} \\
\mathrm{x} \\
\mathrm{x} \\
\mathrm{x} \\
\mathrm{x} \\
\mathrm{x} \\
\mathrm{x} \\
\mathrm{x} \\
\mathrm{x} \\
\mathrm{x} \\
\mathrm{x} \\
\mathrm{x}\end{array}$ & $\begin{array}{l}\mathrm{x} \\
\mathrm{x} \\
\mathrm{x} \\
\mathrm{x} \\
\mathrm{x} \\
\mathrm{x} \\
\mathrm{x} \\
\mathrm{x} \\
\mathrm{x} \\
\mathrm{x} \\
\mathrm{x} \\
\mathrm{x}\end{array}$ & \begin{tabular}{l|}
$\mathrm{x}$ \\
$\mathrm{x}$ \\
$\mathrm{x}$ \\
$\mathrm{x}$ \\
$\mathrm{x}$ \\
$\mathrm{x}$ \\
$\mathrm{x}$ \\
$\mathrm{x}$ \\
$\mathrm{x}$ \\
$\mathrm{x}$ \\
$\mathrm{x}$ \\
$\mathrm{x}$ \\
$\mathrm{x}$
\end{tabular} & & $\begin{array}{ll} & \mathrm{x} \\
\mathrm{x} & \mathrm{x} \\
\mathrm{x} & \\
\mathrm{x} & \mathrm{x} \\
\mathrm{x} & \mathrm{x} \\
\mathrm{x} & \\
\end{array}$ & $\begin{array}{ll} & \mathrm{x} \\
& \mathrm{x} \\
& \mathrm{x} \\
\mathrm{x} & \mathrm{x} \\
& \mathrm{x} \\
& \mathrm{x} \\
\mathrm{x} & \mathrm{x} \\
\mathrm{x} & \mathrm{x} \\
& \mathrm{x} \\
\mathrm{x} & \mathrm{x} \\
\mathrm{x} & \mathrm{x} \\
& \mathrm{x}\end{array}$ & $\begin{array}{ll}\mathrm{x} & \mathrm{x} \\
\mathrm{x} & \mathrm{x} \\
\mathrm{x} & \mathrm{x} \\
& \mathrm{x} \\
\mathrm{x} & \mathrm{x} \\
\mathrm{x} & \mathrm{x} \\
\mathrm{x} & \mathrm{x} \\
\mathrm{x} & \mathrm{x} \\
\mathrm{x} & \mathrm{x} \\
\mathrm{x} & \mathrm{x} \\
\mathrm{x} & \mathrm{x} \\
\mathrm{x} & \mathrm{x} \\
\mathrm{x} & \mathrm{x}\end{array}$ & & $\begin{array}{l}x \\
x \\
x \\
x \\
x \\
x \\
\\
x \\
x \\
x \\
x\end{array}$ & $\begin{array}{l}\mathrm{x} \\
\mathrm{x} \\
\mathrm{x} \\
\mathrm{x} \\
\mathrm{x} \\
\mathrm{x} \\
\mathrm{x} \\
\mathrm{x} \\
\mathrm{x} \\
\mathrm{x} \\
\mathrm{x} \\
\mathrm{x}\end{array}$ & $\begin{array}{l}\mathrm{x} \\
\mathrm{x} \\
\mathrm{x}\end{array}$ & & $\begin{array}{l}x \\
x \\
x \\
x \\
x \\
x \\
x \\
x \\
x \\
x \\
x \\
x \\
x\end{array}$ & $\begin{array}{l}\mathrm{x} \\
\mathrm{x} \\
\mathrm{x} \\
\mathrm{x} \\
\mathrm{x} \\
\mathrm{x} \\
\mathrm{x} \\
\mathrm{x} \\
\mathrm{x} \\
\mathrm{x} \\
\mathrm{x}\end{array}$ & $\begin{array}{l}x \\
x \\
x \\
x \\
x \\
x \\
x \\
x \\
x \\
x \\
x \\
x \\
x\end{array}$ & $\begin{array}{l}\mathrm{x} \\
\mathrm{x} \\
\mathrm{x} \\
\mathrm{x} \\
\mathrm{x} \\
\mathrm{x} \\
\mathrm{x} \\
\mathrm{x} \\
\mathrm{x} \\
\mathrm{x} \\
\mathrm{x} \\
\mathrm{x} \\
\mathrm{x}\end{array}$ & $\begin{array}{l}\mathrm{x} \\
\mathrm{x} \\
\mathrm{x} \\
\mathrm{x} \\
\mathrm{x} \\
\mathrm{x} \\
\mathrm{x} \\
\mathrm{x} \\
\mathrm{x} \\
\mathrm{x} \\
\mathrm{x} \\
\mathrm{x}\end{array}$ & $\begin{array}{l}x \\
x \\
x \\
x \\
x \\
x \\
x \\
x \\
x \\
x \\
x \\
x \\
x\end{array}$ \\
\hline & \multirow[t]{2}{*}{$\begin{array}{l}\text { Discoaster lodoensis Zone } \\
\text { CP11*, NP13** }\end{array}$} & $\begin{array}{l}\text { Rhabdosphaera tenuis } \\
\text { Subzone } \\
\text { CP11*c, NP13*c }\end{array}$ & $\begin{array}{l}43, C C \\
44-1,20-22 \\
44-2,20-22 \\
44-3,20-22 \\
44-4,20-22 \\
44-5,20-22 \\
44-6,20-22 \\
44, C C \\
45-2,20-22 \\
45-4,20-22 \\
45, C C \\
46-3,20-22 \\
46-5,20-22 \\
46, C C \\
47-1,20-22 \\
47-2,20-22\end{array}$ & $\begin{array}{l}395.57 \\
395.81 \\
397.31 \\
398.81 \\
400.31 \\
401.81 \\
403.31 \\
405.17 \\
406.91 \\
409.91 \\
414.32 \\
418.11 \\
421.11 \\
424.45 \\
424.81 \\
426.31\end{array}$ & $\begin{array}{l}\mathrm{x} \\
\mathrm{x}\end{array}$ & $\begin{array}{l}x \\
x \\
x \\
x \\
x \\
x \\
x \\
x \\
x \\
x \\
x \\
x \\
x \\
x \\
x\end{array}$ & $\begin{array}{l}x \\
x \\
\\
x \\
x \\
x \\
x \\
x \\
x \\
x \\
x \\
x\end{array}$ & $\begin{array}{l}\mathrm{x} \\
\mathrm{x} \\
\mathrm{x} \\
\mathrm{x} \\
\mathrm{x} \\
\mathrm{x} \\
\mathrm{x} \\
\mathrm{x} \\
\mathrm{x} \\
\mathrm{x} \\
\mathrm{x} \\
\mathrm{x} \\
\mathrm{x} \\
\mathrm{x}\end{array}$ & $\begin{array}{l}x \\
x \\
x \\
x \\
x \\
x \\
x \\
x \\
\\
x \\
x \\
x \\
x \\
x \\
x \\
x\end{array}$ & $\begin{array}{l}\mathrm{x} \\
\mathrm{x} \\
\mathrm{x} \\
\mathrm{x} \\
\mathrm{x} \\
\mathrm{x} \\
\mathrm{x} \\
\mathrm{x} \\
\mathrm{x} \\
\mathrm{x} \\
\mathrm{x} \\
\mathrm{x} \\
\mathrm{x} \\
\mathrm{x} \\
\mathrm{x} \\
\mathrm{x}\end{array}$ & $\begin{array}{l}\mathrm{x} \\
\mathrm{x} \\
\mathrm{x} \\
\mathrm{x} \\
\mathrm{x} \\
\mathrm{x} \\
\mathrm{x} \\
\mathrm{x} \\
\mathrm{x} \\
\mathrm{x} \\
\mathrm{x} \\
\mathrm{x} \\
\mathrm{x} \\
\mathrm{x} \\
\mathrm{x} \\
\mathrm{x}\end{array}$ & $\begin{array}{l}\mathrm{x} \\
\mathrm{x} \\
\mathrm{x} \\
\mathrm{x} \\
\mathrm{x} \\
\mathrm{x} \\
\mathrm{x} \\
\mathrm{x} \\
\mathrm{x} \\
\mathrm{x} \\
\mathrm{x} \\
\mathrm{x} \\
\mathrm{x} \\
\mathrm{x} \\
\mathrm{x} \\
\mathrm{x}\end{array}$ & & $\begin{array}{ll}x & \\
& x \\
x & x \\
x & \\
& x \\
x & \\
x & \\
x & x \\
x & \\
x & \\
x & \\
x & \end{array}$ & $\begin{array}{ll}x & x \\
& x \\
x & x \\
& x \\
& x \\
x & x \\
x & x \\
& x \\
x & x \\
& x \\
& x \\
x & x \\
& x \\
x & x \\
& x \\
& x\end{array}$ & $\begin{array}{ll}\mathrm{x} & \mathrm{x} \\
\mathrm{x} & \mathrm{x} \\
\mathrm{x} & \mathrm{x} \\
\mathrm{x} & \mathrm{x} \\
\mathrm{x} & \mathrm{x} \\
\mathrm{x} & \mathrm{x} \\
\mathrm{x} & \mathrm{x} \\
\mathrm{x} & \mathrm{x} \\
\mathrm{x} & \mathrm{x} \\
\mathrm{x} & \mathrm{x} \\
\mathrm{x} & \mathrm{x} \\
\mathrm{x} & \mathrm{x} \\
\mathrm{x} & \mathrm{x} \\
\mathrm{x} & \mathrm{x} \\
\mathrm{x} & \mathrm{x} \\
\mathrm{x} & \mathrm{x}\end{array}$ & & $\begin{array}{l}x \\
x \\
x \\
x \\
x \\
x \\
x \\
x \\
x \\
x \\
x \\
x \\
x \\
x\end{array}$ & $\begin{array}{l}\mathrm{x} \\
\mathrm{x} \\
\mathrm{x} \\
\mathrm{x} \\
\mathrm{x} \\
\mathrm{x} \\
\mathrm{x} \\
\mathrm{x} \\
\mathrm{x} \\
\mathrm{x} \\
\mathrm{x} \\
\mathrm{x} \\
\mathrm{x} \\
\mathrm{x} \\
\mathrm{x} \\
\mathrm{x}\end{array}$ & \begin{tabular}{l|}
$\mathrm{x}$ \\
$\mathrm{x}$ \\
$\mathrm{x}$ \\
$\mathrm{x}$ \\
$\mathrm{x}$ \\
$\mathrm{x}$ \\
$\mathrm{x}$ \\
$\mathrm{x}$ \\
$\mathrm{x}$ \\
$\mathrm{x}$ \\
$\mathrm{x}$ \\
$\mathrm{x}$ \\
$\mathrm{x}$
\end{tabular} & & $\begin{array}{l}\mathrm{x} \\
\mathrm{x} \\
\mathrm{x} \\
\mathrm{x} \\
\mathrm{x} \\
\mathrm{x} \\
\mathrm{x} \\
\mathrm{x} \\
\mathrm{x} \\
\mathrm{x} \\
\mathrm{x} \\
\mathrm{x} \\
\mathrm{x} \\
\mathrm{x} \\
\mathrm{x} \\
\mathrm{x}\end{array}$ & $\begin{array}{l}x \\
x \\
x \\
x \\
x \\
x \\
x \\
x \\
x \\
x \\
x \\
x \\
x \\
x \\
x \\
\end{array}$ & $\begin{array}{l}x \\
x \\
x \\
x \\
x \\
x \\
x \\
x \\
x \\
x \\
x \\
x \\
x \\
x \\
x \\
x\end{array}$ & & $\begin{array}{l}x \\
x \\
x \\
x \\
x \\
x \\
x \\
x \\
x \\
x \\
x \\
x \\
x \\
x \\
x \\
x\end{array}$ & $\begin{array}{l}x \\
x \\
x \\
x \\
x \\
x \\
x \\
x \\
x \\
x \\
x \\
x \\
x \\
x \\
x \\
x\end{array}$ \\
\hline & & $\begin{array}{c}\text { Cyclicargolithus } \\
\text { pseudogammation Subzone } \\
\text { CP11*b, NP13*b }\end{array}$ & $\begin{array}{l}47-3,20-22 \\
47-4,20-22 \\
47-5,20-22 \\
47-6,20-22 \\
47, \mathrm{CC}\end{array}$ & $\begin{array}{l}427.81 \\
429.31 \\
430.81 \\
432.31 \\
434.24\end{array}$ & $x$ & $\begin{array}{l}\mathrm{x} \\
\mathrm{x} \\
\mathrm{x} \\
\mathrm{x} \\
\mathrm{x}\end{array}$ & $\begin{array}{l}x \\
x \\
x \\
x \\
x\end{array}$ & $\begin{array}{l}x \\
x \\
x\end{array}$ & $\begin{array}{l}x \\
x \\
x\end{array}$ & $\begin{array}{l}\mathrm{x} \\
\mathrm{x} \\
\mathrm{x} \\
\mathrm{x} \\
\mathrm{x}\end{array}$ & $\begin{array}{l}\mathrm{x} \\
\mathrm{x} \\
\mathrm{x}\end{array}$ & $\begin{array}{l}\mathrm{x} \\
\mathrm{x} \\
\mathrm{x} \\
\mathrm{x} \\
\mathrm{x}\end{array}$ & & $\begin{array}{ll}x & x \\
x & x\end{array}$ & $\begin{array}{ll}x & x \\
& x \\
x & x \\
x & x \\
x\end{array}$ & $\begin{array}{ll}\mathrm{x} & \mathrm{x} \\
\mathrm{x} & \mathrm{x} \\
\mathrm{x} & \mathrm{x} \\
\mathrm{x} & \mathrm{x} \\
\mathrm{x} & \mathrm{x}\end{array}$ & & $\begin{array}{l}x \\
x \\
x \\
x\end{array}$ & $\begin{array}{l}\mathrm{x} \\
\mathrm{x} \\
\mathrm{x} \\
\mathrm{x}\end{array}$ & $\begin{array}{l}\mathrm{x} \\
\mathrm{x} \\
\mathrm{x}\end{array}$ & & $\begin{array}{l}\mathrm{x} \\
\mathrm{x} \\
\mathrm{x} \\
\mathrm{x} \\
\mathrm{x}\end{array}$ & $\begin{array}{l}x \\
x \\
x \\
x \\
x\end{array}$ & $\begin{array}{l}x \\
x \\
x\end{array}$ & & & $\begin{array}{l}\mathrm{x} \\
\mathrm{x} \\
\mathrm{x} \\
\mathrm{x} \\
\mathrm{x}\end{array}$ \\
\hline
\end{tabular}




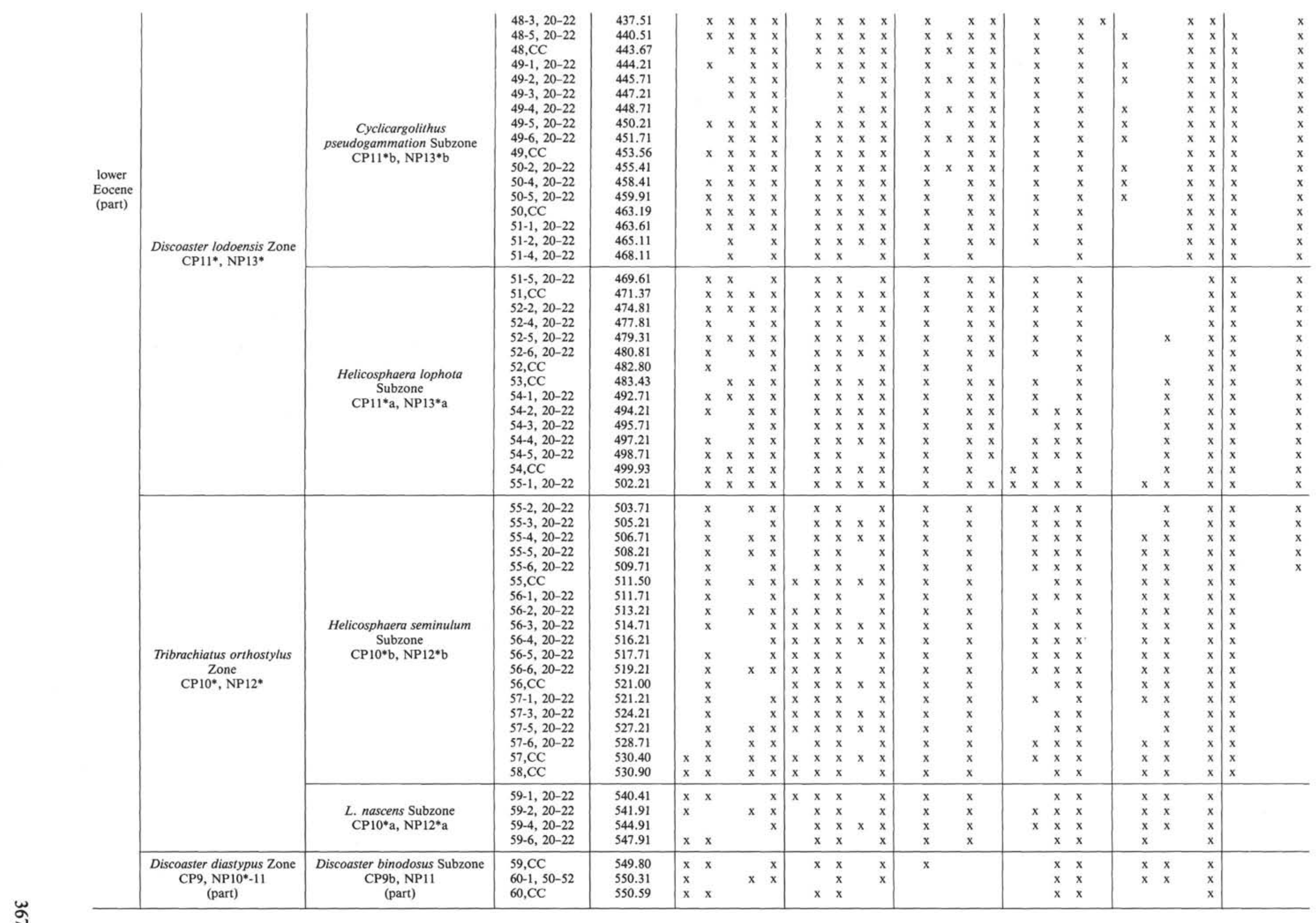


Table 2. Ranges of selected nannofossil species, Site 613, New Jersey rise.

\begin{tabular}{|c|c|c|c|c|c|c|c|c|c|c|c|c|c|c|c|c|c|c|c|c|c|c|c|c|c|c|c|}
\hline \multirow{2}{*}{$\begin{array}{l}\text { Series } \\
\text { middle } \\
\begin{array}{l}\text { Eocene } \\
\text { (part) }\end{array}\end{array}$} & \multicolumn{2}{|c|}{$\begin{array}{c}\text { Biozone | } \\
\text { (Valentine, this study) }\end{array}$} & \multirow{2}{*}{$\begin{array}{l}\text { Core-Section, } \\
\text { interval }(\mathrm{cm})\end{array}$} & \multirow{2}{*}{$\begin{array}{c}\begin{array}{c}\text { Sub-bottom } \\
\text { depth } \\
\text { (m) }\end{array} \\
430.50 \\
438.80 \\
439.20 \\
439.30\end{array}$} & \multicolumn{2}{|c|}{ 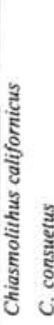 } & \multicolumn{2}{|c|}{ 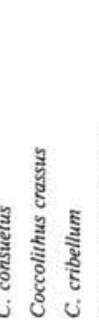 } & \multicolumn{3}{|c|}{ 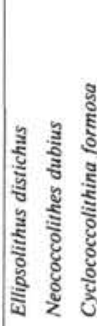 } & & 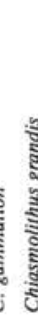 & 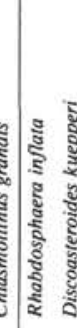 & 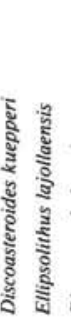 & 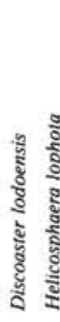 & & 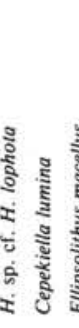 & \multicolumn{2}{|c|}{ 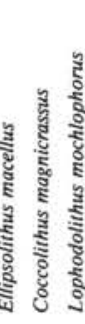 } & \multicolumn{2}{|c|}{ 赔 } & \multicolumn{2}{|c|}{ 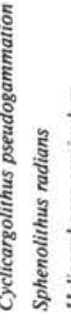 } & \multicolumn{3}{|c|}{ 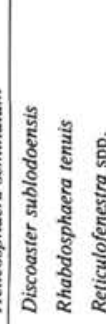 } \\
\hline & & $\begin{array}{c}\text { R. inflata } \mathrm{Sz} . \\
\mathrm{CP} 12^{*}{ }^{*}, \mathrm{NP}^{*} 4^{\circ} \mathrm{b} \\
\text { (part) }\end{array}$ & & & & $\begin{array}{l}x \\
x \\
x \\
x\end{array}$ & & $\begin{array}{r}\mathrm{x} \\
\mathrm{x} \\
\mathrm{x} \\
\mathrm{x}\end{array}$ & & $\begin{array}{l}x \\
x \\
x \\
x\end{array}$ & $\begin{array}{l}\mathrm{x} \\
\mathrm{x} \\
\mathrm{x} \\
\mathrm{x}\end{array}$ & $\begin{array}{l}x \\
x\end{array}$ & $\begin{array}{l}\mathrm{x} \\
\mathrm{x} \\
\mathrm{x} \\
\mathrm{x}\end{array}$ & $\begin{array}{l}x \\
x \\
x \\
x\end{array}$ & & $\begin{array}{ll} & x \\
x & x \\
x & x\end{array}$ & & $\begin{array}{l}x \\
x \\
x\end{array}$ & & $\begin{array}{l}\mathrm{x} \\
\mathrm{x}\end{array}$ & & & & $\begin{array}{ll}\mathrm{x} & \mathrm{x} \\
\mathrm{x} & \mathrm{x} \\
\mathrm{x} & \mathrm{x} \\
\mathrm{x} & \mathrm{x}\end{array}$ & & $\begin{array}{l}x \\
x \\
x \\
x\end{array}$ & \\
\hline \multirow{6}{*}{$\begin{array}{l}\text { lower } \\
\text { Eocene } \\
\text { (part) }\end{array}$} & $\begin{array}{c}\text { Discoaster } \\
\text { sublodoensis } \\
\text { Zone } \\
\text { CP12, NP14* } \\
\text { (part) }\end{array}$ & $\begin{array}{l}\text { D. kuepperi Subzone } \\
\text { CP1 } 2^{*} \mathrm{a}, \mathrm{NP1} 4^{*}{ }^{\circ}\end{array}$ & $\begin{array}{l}37, \mathrm{CC} \\
38-1,90-91 \\
38-3,40 \\
38-4,6 \\
38, \mathrm{CC} \\
39-6,51 \\
40, \mathrm{CC} \\
41-1,90-91 \\
41-3,90-91 \\
41-4,90-91\end{array}$ & $\begin{array}{l}439.70 \\
440.91 \\
443.40 \\
444.56 \\
445.52 \\
457.51 \\
459.55 \\
469.41 \\
472.41 \\
473.91\end{array}$ & & $\begin{array}{l}\mathrm{x} \\
\mathrm{x} \\
\mathrm{x} \\
\mathrm{x} \\
\mathrm{x}\end{array}$ & $\begin{array}{ll} & \mathrm{x} \\
\mathrm{x} & \\
\mathrm{x} & \end{array}$ & $\begin{array}{ll}\mathrm{x} & \mathrm{x} \\
\mathrm{x} \\
\mathrm{x} \\
\mathrm{x} \\
\mathrm{x} \\
\mathrm{x} \\
\mathrm{x}\end{array}$ & & $\begin{array}{l}\mathrm{x} \\
\mathrm{x} \\
\mathrm{x} \\
\mathrm{x} \\
\mathrm{x} \\
\mathrm{x} \\
\mathrm{x} \\
\mathrm{x} \\
\mathrm{x}\end{array}$ & $\begin{array}{l}x \\
x \\
x \\
x \\
x \\
x \\
x \\
x \\
\\
x \\
x \\
x\end{array}$ & $\begin{array}{l}\mathrm{x} \\
\mathrm{x} \\
\mathrm{x} \\
\mathrm{x} \\
\mathrm{x} \\
\mathrm{x} \\
\mathrm{x} \\
\mathrm{x} \\
\mathrm{x} \\
\mathrm{x}\end{array}$ & \begin{tabular}{l|l}
$x$ \\
$x$ \\
$x$ \\
$x$ \\
$x$ \\
$x$ \\
$x$ \\
$x$ \\
$x$ \\
$x$ \\
$x$
\end{tabular} & & $\begin{array}{ll} & x \\
& x \\
x & x \\
x & \\
& x\end{array}$ & $\begin{array}{ll}\mathrm{x} & \mathrm{x} \\
\mathrm{x} & \mathrm{x} \\
\mathrm{x} & \mathrm{x} \\
\mathrm{x} & \mathrm{x} \\
\mathrm{x} & \mathrm{x} \\
\mathrm{x} & \mathrm{x} \\
\mathrm{x} & \\
\mathrm{x} & \mathrm{x} \\
\mathrm{x} & \mathrm{x} \\
\mathrm{x} & \end{array}$ & & $\begin{array}{l}x \\
x \\
x \\
x \\
x \\
x\end{array}$ & $\begin{array}{l}\mathrm{x} \\
\mathrm{x}\end{array}$ & $\begin{array}{r}\mathrm{x} \\
\mathrm{x} \\
\mathrm{x} \\
\mathrm{x}\end{array}$ & & $x$ & $\begin{array}{l}\mathrm{x} \\
\mathrm{x} \\
\mathrm{x} \\
\mathrm{x} \\
\mathrm{x} \\
\mathrm{x} \\
\mathrm{x} \\
\mathrm{x} \\
\mathrm{x} \\
\mathrm{x} \\
\mathrm{x}\end{array}$ & $\begin{array}{ll}\mathrm{x} & \mathrm{x} \\
\mathrm{x} & \mathrm{x} \\
\mathrm{x} & \mathrm{x} \\
\mathrm{x} & \mathrm{x} \\
\mathrm{x} & \mathrm{x} \\
\mathrm{x} & \mathrm{x} \\
\mathrm{x} & \mathrm{x} \\
\mathrm{x} & \mathrm{x}\end{array}$ & & $\begin{array}{l}x \\
x \\
x \\
x \\
x \\
x \\
x \\
x \\
x \\
x\end{array}$ & $\begin{array}{l}\mathrm{x} \\
\mathrm{x} \\
\mathrm{x} \\
\mathrm{x} \\
\mathrm{x} \\
\mathrm{x} \\
\mathrm{x} \\
\mathrm{x} \\
\mathrm{x} \\
\mathrm{x}\end{array}$ \\
\hline & \multirow{2}{*}{$\begin{array}{c}\text { Discoaster } \\
\text { iodoensis Zone } \\
\text { CP11 }\end{array}$} & $\begin{array}{c}\text { R. tenuis } \\
\text { Subzone } \\
\text { CP11*c, NP13*c }\end{array}$ & $\begin{array}{l}41-5,20-21 \\
41-5,90-91 \\
41-6,20-21 \\
42, \mathrm{CC}-2 \\
43-1,20-21 \\
43-2,90-91 \\
43-4,20-21 \\
43, \mathrm{CC}\end{array}$ & $\begin{array}{l}474.71 \\
475.41 \\
476.21 \\
478.42 \\
487.30 \\
489.51 \\
491.80 \\
494.83\end{array}$ & & $x$ & $\begin{array}{l}x \\
x \\
x\end{array}$ & $\begin{array}{l}x \\
x \\
x \\
x \\
x \\
x \\
x\end{array}$ & & $\begin{array}{l}x \\
x \\
x \\
x \\
x \\
x \\
x \\
x\end{array}$ & $\begin{array}{l}\mathrm{x} \\
\mathrm{x} \\
\mathrm{x} \\
\mathrm{x} \\
\mathrm{x} \\
\mathrm{x} \\
\mathrm{x} \\
\mathrm{x} \\
\end{array}$ & $\begin{array}{l}\mathrm{x} \\
\mathrm{x} \\
\mathrm{x} \\
\mathrm{x} \\
\mathrm{x} \\
\mathrm{x} \\
\mathrm{x} \\
\mathrm{x}\end{array}$ & \begin{tabular}{l|l|}
$x$ \\
$x$ \\
$x$ \\
$x$ \\
$x$ \\
$x$ \\
$x$ \\
$x$ \\
$x$
\end{tabular} & $\begin{array}{l}x \\
x \\
x \\
x\end{array}$ & $\begin{array}{ll}x & \\
& x \\
x & x \\
x & x \\
x & x \\
x & \\
\end{array}$ & $\begin{array}{ll}\mathrm{x} & \\
\mathrm{x} & \mathrm{x} \\
\mathrm{x} & \mathrm{x} \\
\mathrm{x} & \\
\mathrm{x} & \mathrm{x} \\
\mathrm{x} & \mathrm{x} \\
\mathrm{x} & \mathrm{x} \\
\mathrm{x} & \mathrm{x}\end{array}$ & & $\begin{array}{l}x \\
x \\
x \\
x \\
x \\
\\
x \\
x\end{array}$ & $\begin{array}{l}x \\
x \\
x \\
x \\
x \\
x\end{array}$ & $\begin{array}{ll}x & \\
x & x \\
x & \\
x & \\
x & \\
x & \end{array}$ & & $\begin{array}{l}\mathrm{x} \\
\mathrm{x} \\
\mathrm{x}\end{array}$ & $\begin{array}{l}x \\
x \\
x \\
x \\
x \\
x \\
x \\
x \\
x\end{array}$ & $\begin{array}{ll}x & \\
x & x \\
x & \\
& x \\
x & \\
x & \\
x & x\end{array}$ & & $\begin{array}{l}x \\
x \\
x \\
x\end{array}$ & \\
\hline & & $\begin{array}{c}\text { H. lophota } \\
\text { Subzone } \\
\text { CP11*a, NP13*a }\end{array}$ & $\begin{array}{l}44-1,20-21 \\
44-3,20-21 \\
44-4,20-21 \\
44-5,20-21 \\
44-6,20-21 \\
44, C C \\
45-1,90-91\end{array}$ & $\begin{array}{l}-796.61 \\
499.61 \\
501.11 \\
502.61 \\
504.11 \\
505.90 \\
506.81\end{array}$ & & $x$ & $\begin{array}{ll}x & \\
x & x \\
x & x \\
x & x \\
& x \\
& x \\
& x \\
x & x\end{array}$ & $\begin{array}{lll}x & & \\
x \\
x & x \\
x & x \\
x & x \\
x & x \\
x & x \\
x & x\end{array}$ & & $\begin{array}{l}x \\
x \\
x \\
x \\
x \\
x \\
x\end{array}$ & $\begin{array}{l}\bar{x} \\
x \\
x \\
x \\
x \\
x \\
x\end{array}$ & $\begin{array}{l}\bar{x} \\
x \\
x \\
x \\
x \\
x \\
x\end{array}$ & $\begin{array}{l}x \\
x \\
x \\
x \\
x \\
x \\
x \\
x\end{array}$ & $\begin{array}{l}x \\
x \\
x \\
x \\
x \\
x \\
x \\
x\end{array}$ & $\begin{array}{l}x \\
x \\
x \\
x \\
x \\
x \\
x \\
x\end{array}$ & $\begin{array}{ll} & x \\
x & x \\
x & x \\
x & x \\
x & x \\
x & \\
x & x \\
x & x\end{array}$ & $\begin{array}{l}x \\
x \\
x \\
x\end{array}$ & $\begin{array}{l}x \\
x \\
x\end{array}$ & $\begin{array}{ll} & \mathrm{x} \\
& \mathrm{x} \\
\mathrm{x} & \mathrm{x} \\
\mathrm{x} & \mathrm{x} \\
& \mathrm{x} \\
\mathrm{x} & \mathrm{x} \\
& \mathrm{x}\end{array}$ & $\begin{array}{l}x \\
x \\
x \\
x \\
x \\
x \\
x \\
x \\
\end{array}$ & $\mathrm{x}$ & $\begin{array}{l}\mathrm{x} \\
\mathrm{x} \\
\mathrm{x} \\
\mathrm{x} \\
\mathrm{x} \\
\mathrm{x} \\
\mathrm{x}\end{array}$ & & $\begin{array}{ll}x & x \\
x & x \\
x & x \\
x & x \\
x & x \\
x & x \\
x & x \\
x & x \\
& x\end{array}$ & & & $\begin{array}{l}x \\
x \\
x \\
x \\
x \\
x \\
x \\
x\end{array}$ \\
\hline & \multirow[t]{2}{*}{$\begin{array}{c}\text { Tribrachiatus } \\
\text { orthostylus } \\
\text { Zone } \\
\text { CP10*, NP12* }\end{array}$} & $\begin{array}{l}\text { H. seminulum } \\
\text { Subzone } \\
\text { CP10*b, NP12*b }\end{array}$ & $\begin{array}{l}45-2,90-91 \\
45-3,90-91 \\
45-4,90-91 \\
45-4,90-91 \\
45, C C \\
46-1,20-21 \\
46-1,90-91 \\
46-3,90-91 \\
46-5,20-21 \\
46-5,90-91 \\
46-6,90-91 \\
46, C C\end{array}$ & $\begin{array}{l}508.31 \\
509.81 \\
511.31 \\
512.81 \\
514.33 \\
515.60 \\
516.31 \\
519.31 \\
521.61 \\
522.31 \\
523.81 \\
524.90\end{array}$ & & $\begin{array}{l}\mathrm{x} \\
\mathrm{x} \\
\mathrm{x} \\
\mathrm{x} \\
\mathrm{x} \\
\mathrm{x} \\
\mathrm{x} \\
\mathrm{x} \\
\mathrm{x}\end{array}$ & $\begin{array}{l}\mathrm{x} \\
\mathrm{x} \\
x \\
\mathrm{x} \\
\mathrm{x} \\
\mathrm{x}\end{array}$ & $\begin{array}{ll}\mathrm{x} & \\
\mathrm{x} & \mathrm{x} \\
\mathrm{x} & \mathrm{x} \\
\mathrm{x} & \mathrm{x} \\
\mathrm{x} & \mathrm{x} \\
\mathrm{x} & \mathrm{x} \\
& \mathrm{x} \\
& \mathrm{x} \\
& \mathrm{x} \\
\mathrm{x} & \mathrm{x} \\
& \mathrm{x} \\
& \mathrm{x}\end{array}$ & $\begin{array}{l}x \\
x \\
x\end{array}$ & $\begin{array}{l}\mathrm{x} \\
\mathrm{x} \\
\mathrm{x} \\
\mathrm{x} \\
\mathrm{x} \\
\mathrm{x} \\
\mathrm{x} \\
\mathrm{x} \\
\mathrm{x} \\
\mathrm{x} \\
\mathrm{x} \\
\mathrm{x}\end{array}$ & $\begin{array}{l}\mathrm{x} \\
\mathrm{x} \\
\mathrm{x} \\
\mathrm{x} \\
\mathrm{x} \\
\mathrm{x} \\
\mathrm{x} \\
\mathrm{x} \\
\mathrm{x} \\
\mathrm{x} \\
\mathrm{x} \\
\mathrm{x}\end{array}$ & $\begin{array}{l}\mathrm{x} \\
\mathrm{x} \\
\mathrm{x}\end{array}$ & $\begin{array}{l}x \\
x \\
x \\
x \\
x \\
x \\
x \\
x \\
x \\
x \\
x \\
x \\
x\end{array}$ & $\begin{array}{l}x \\
x \\
x \\
x \\
x \\
x \\
x \\
x \\
x \\
x \\
x \\
x \\
x\end{array}$ & $\begin{array}{l}\mathbf{x} \\
x \\
x \\
x \\
x \\
x \\
x \\
x \\
x \\
x \\
x \\
x \\
x\end{array}$ & $\begin{array}{l}\mathrm{x} \\
\mathrm{x} \\
\mathrm{x} \\
\mathrm{x} \\
\mathrm{x} \\
\mathrm{x} \\
\mathrm{x} \\
\mathrm{x} \\
\mathrm{x} \\
\mathrm{x} \\
\mathrm{x} \\
\mathrm{x}\end{array}$ & $\begin{array}{l}x \\
x \\
x\end{array}$ & $\begin{array}{l}\mathrm{x} \\
\mathrm{x} \\
\mathrm{x} \\
\mathrm{x} \\
\mathrm{x} \\
\mathrm{x} \\
\mathrm{x}\end{array}$ & $\begin{array}{ll}\mathrm{x} & \mathrm{x} \\
\mathrm{x} & \mathrm{x} \\
\mathrm{x} & \mathrm{x} \\
\mathrm{x} & \mathrm{x} \\
\mathrm{x} & \mathrm{x} \\
& \mathrm{x} \\
& \mathrm{x} \\
& \mathrm{x} \\
\mathrm{x} & \mathrm{x} \\
& \mathrm{x} \\
& \mathrm{x} \\
\mathrm{x} & \mathrm{x}\end{array}$ & $\begin{array}{l}\mathrm{x} \\
\mathrm{x} \\
\mathrm{x} \\
\mathrm{x} \\
\mathrm{x} \\
\mathrm{x} \\
\mathrm{x} \\
\mathrm{x} \\
\mathrm{x} \\
\mathrm{x} \\
\mathrm{x} \\
\mathrm{x}\end{array}$ & $\begin{array}{l}x \\
x \\
x \\
x \\
x \\
x \\
x \\
x \\
x \\
x\end{array}$ & $\begin{array}{l}\mathrm{x} \\
\mathrm{x} \\
\mathrm{x} \\
\mathrm{x} \\
\mathrm{x} \\
\mathrm{x} \\
\mathrm{x} \\
\mathrm{x} \\
\mathrm{x} \\
\mathrm{x} \\
\mathrm{x} \\
\mathrm{x} \\
\end{array}$ & & $\begin{array}{ll}\mathrm{x} & \mathrm{x} \\
\mathrm{x} & \mathrm{x} \\
\mathrm{x} & \\
\mathrm{x} & \mathrm{x} \\
\mathrm{x} & \mathrm{x} \\
\mathrm{x} & \mathrm{x} \\
\mathrm{x} & \mathrm{x} \\
\mathrm{x} & \mathrm{x} \\
\mathrm{x} & \\
\mathrm{x} & \mathrm{x} \\
\mathrm{x} & \mathrm{x} \\
\mathrm{x} & \mathrm{x}\end{array}$ & & & $\begin{array}{l}x \\
x \\
x \\
x \\
x \\
x\end{array}$ \\
\hline & & $\begin{array}{c}\text { L. nascens } \\
\text { Subzone } \\
\text { CP10*a, NP12*a }\end{array}$ & $\begin{array}{l}47, \mathrm{CC} \\
48-1,20-21 \\
48-1,90-91 \\
48, \mathrm{CC} \\
49, \mathrm{CC} \\
50-1,20-21 \\
50-3,90-91 \\
50-4,90-91\end{array}$ & $\begin{array}{l}525.34 \\
534.60 \\
535.30 \\
535.94 \\
544.10 \\
553.60 \\
557.31 \\
558.81\end{array}$ & \begin{tabular}{|l}
$x$ \\
$x$ \\
$x$ \\
$x$
\end{tabular} & $\begin{array}{l}x \\
x \\
x \\
x \\
x \\
x\end{array}$ & $\begin{array}{l}x \\
x \\
x \\
x\end{array}$ & $\begin{array}{ll}x & x \\
x & \\
x & x \\
& x\end{array}$ & & $\begin{array}{l}x \\
x \\
x \\
x \\
x \\
x \\
x \\
x\end{array}$ & $\begin{array}{l}\mathrm{x} \\
\mathrm{x} \\
\mathrm{x} \\
\mathrm{x} \\
\mathrm{x} \\
\mathrm{x} \\
\mathrm{x}\end{array}$ & $\mathrm{x}$ & $\begin{array}{l}x \\
x \\
x \\
x \\
x \\
x \\
x\end{array}$ & $\begin{array}{l}x \\
x \\
x \\
x \\
x \\
x \\
x \\
x \\
x\end{array}$ & $\begin{array}{l}x \\
x \\
x \\
x \\
x \\
x \\
x \\
x \\
x\end{array}$ & $\begin{array}{l}\mathrm{x} \\
\mathrm{x} \\
\mathrm{x} \\
\mathrm{x} \\
\mathrm{x} \\
\mathrm{x} \\
\mathrm{x} \\
\mathrm{x} \\
\mathrm{x}\end{array}$ & & $\begin{array}{l}\times \\
\times \\
\end{array}$ & $\begin{array}{ll} & \mathrm{x} \\
\mathrm{x} & \mathrm{x} \\
& \mathrm{x} \\
\mathrm{x} & \mathrm{x} \\
\mathrm{x} & \mathrm{x} \\
\mathrm{x} & \mathrm{x} \\
\mathrm{x} & \mathrm{x} \\
\end{array}$ & $\begin{array}{l}x \\
x \\
x \\
x \\
x \\
x \\
x \\
x\end{array}$ & $\begin{array}{l}x \\
x \\
x \\
x\end{array}$ & $\begin{array}{l}\mathrm{x} \\
\mathrm{x} \\
\mathrm{x} \\
\mathrm{x} \\
\mathrm{x} \\
\mathrm{x} \\
\mathrm{x} \\
\mathrm{x}\end{array}$ & & $\begin{array}{l}x \\
x \\
x \\
x \\
x \\
x \\
x \\
x \\
x \\
x\end{array}$ & & & \\
\hline & $\begin{array}{l}\text { Discoaster } \\
\text { diastypus Zone } \\
\text { CP9, NP10*-11 } \\
\text { (part) }\end{array}$ & $\begin{array}{l}\text { D. binodosus } \\
\text { Subzone } \\
\text { CP9g, NP11 } \\
\text { (part) }\end{array}$ & $\begin{array}{l}50-5,90-91 \\
50-6,20-21 \\
50, C C \\
51-3,20-21 \\
51, \mathrm{CC} \\
52, \mathrm{CC}\end{array}$ & $\begin{array}{l}560.31 \\
561.10 \\
561.54 \\
566.10 \\
572.40 \\
578.06\end{array}$ & $\begin{array}{l}\mathrm{x} \\
\mathrm{x}\end{array}$ & $\begin{array}{l}\mathrm{x} \\
\mathrm{x} \\
\mathrm{x} \\
\mathrm{x}\end{array}$ & & $\begin{array}{l}x \\
x \\
x\end{array}$ & & $\begin{array}{l}x \\
x \\
x \\
x\end{array}$ & $\begin{array}{l}\mathrm{x} \\
\mathrm{x}\end{array}$ & $\begin{array}{l}x \\
x \\
x\end{array}$ & $\hat{a}$ & $\begin{array}{l}x \\
x \\
x \\
x \\
x\end{array}$ & $\begin{array}{l}\mathbf{x} \\
x \\
x\end{array}$ & & & $x$ & $\begin{array}{ll}x & x \\
x & x \\
x & x \\
x & x \\
x & x \\
x & x\end{array}$ & $\begin{array}{l}x \\
x \\
x \\
x \\
x \\
x \\
x\end{array}$ & $\begin{array}{l}x \\
x \\
x \\
x \\
x\end{array}$ & $\begin{array}{l}\mathrm{x} \\
\mathrm{x} \\
\mathrm{x} \\
\mathrm{x} \\
\mathrm{x} \\
\mathrm{x}\end{array}$ & & $\begin{array}{l}x \\
x \\
x \\
x \\
x \\
x\end{array}$ & & & \\
\hline
\end{tabular}

coenura and $R$. dictyoda also are not distinctive enough to be reliable markers. The initial appearance of the genus in Zone CP10* (NP12*) may prove however, to be an important datum.

Cyclicargolithus pseudogammation first appears at Site 612 in Sample 612-51-4, 20-22 cm (468.11 m), in the lower part of CP11* (NP13*), and ranges up through the lower Eocene. At Site 612 it first occurs somewhat above the last occurrence of $T$. orthostylus (excluding a reworked specimen at $434.24 \mathrm{~m}$; see Fig. 4, Table 1); but at Site 613, it overlaps the range of $T$. orthostylus. This could indicate some upward reworking of T. orthostylus at Site 613 , because it is rare and sometimes poorly preserved in its last few occurrences in this section, and specimens at 459.55 and $440.91 \mathrm{~m}$ clearly are reworked (Fig. 5, Table 2). An additional complication in interpreting the section at Site 613 is an unconformity below the first appearance of $C$. pseudogammation that is revealed by comparison of species ranges here with those at Site 612. In the Anglo-Paris Basin, Aubry (1983) reported the first observation of C. pseudogammation to be in the upper part of Zone NP14 (CP12b). Bukry (1973) first mentioned it, however, as part of the CP11 assemblage in his zonation; and Bukry et al., (1977) list- 
ed it in an assemblage (Mf2050) from the Lucia Mudstone (California) that was assigned to $\mathrm{CP} 10$ but is most likely $\mathrm{CP} 11$, and in an assemblage (Mf1225) from the Juncal Formation (California) assigned to Zone CP11. Cyclicargolithus pseudogammation has not been recorded in many of the previous studies on the lower Eocene (Bramlette and Sullivan, 1961; Mohler and Hay, 1967; Gartner, 1971; Martini, 1971; Romein, 1979; Bybell and Gibson, 1985), although it is a distinctive form. The first occurrence of C. pseudogammation is the datum separating the newly defined subzones CP11*a and CP11*b (NP13*a and NP13*b).

Rhabdosphaera tenuis first appears at Site 612 in Sample 612-47-2, 20-22 cm (426.31 m), in Zone CP11* (NP13*) and above the first appearance of C. pseudogammation. It ranges up through the lower Eocene, occurring in almost all samples studied above its first occurrence. At Site 613, the first appearance of $R$. tenuis is in Sample 613-43,CC (494.83 m), also in Zone CP11* (NP13*). However, it is coeval with the first appearance of $C$. pseudogammation and is thus separated by an unconformity from the strata below. The stratigraphic range of $R$. tenuis has not been fully delineated by previous workers, and it was not listed in the zonal assemblages of either Martini (1971) or Bukry (1973). In some cases its first occurrence was reported in strata younger than those in which it occurs at Site 612. In the Anglo-Paris Basin it first appears in Zone NP14 (CP12) (Aubry, 1983), as it does in the Tallahatta Formation of Georgia and Alabama (Bybell and Gibson, 1985). In California it was reported from the Domengine Formation (Unit 4 of Bramlette and Sullivan, 1961), which is assignable to Subzone CP12a, but it was not recorded in the underlying Lodo Formation (Unit 3), which is chiefly CP10 and CP11. $R$. tenuis was listed in an assemblage from the Lucia Mudstone (Mf2050) of California which was assigned to CP10 by Bukry et al. (1977). As mentioned previously, however, results of the present study at Site 612 suggest that this Lucia Mudstone assemblage may actually belong to Zone CP11, because it also contains $C$. pseudogammation, $H$. lophota, $R$. dictyoda, and lacks $T$. orthostylus. R. tenuis was listed also as occurring in an unnamed shale (Mf2051) in the Santa Teresa Hills (California) that was assigned to Zone CP11 by Bukry et al. (1977). Until now, $R$. tenuis has not attracted the attention of workers for use as a marker, but it is a distinctive species and its lower range at Site 612 is well defined. The first occurrence of $R$. tenuis is the datum separating the newly defined Subzones CP11*b and CP11*c (NP13*b and NP13*c).

Ellipsolithus distichus is first observed at Site 612 in Sample 612-59-1, 20-22 (540.41 m), within the lower part of Zone CP10* (NP12*) near the first appearance of $H$. seminulum, and it is present up to sample 612-55,CC $(511.50 \mathrm{~m})$. It is not present in Subzone CP9b (NP11), the lowest Eocene recovered here, but it is known from earlier studies to occur within the upper Paleocene. It disappears in Zone CP10* (NP12*) at a level above the first occurrence of $H$. seminulum and near the first occurrence of the genus Reticulofenestra. The same general relationship is observed at Site 613. The first occur- rence of $E$. distichus in the lower Eocene of Sites 612 and 613 is of no significance, because the work of other investigators has shown it to be a typical constituent of upper Paleocene assemblages. Mohler and Hay (1967) listed it in upper Paleocene to lowest Eocene zones equivalent to NP8, NP9, and NP10. Martini (1971) listed in assemblages of Zones NP5, NP8, and NP9, and depicted it occurring commonly in upper Paleocene Zones NP5 to NP9 (CP4-CP8), with probable sporadic occurrence in lower Eocene Zones NP10 to NP12 (CP9-CP10). Aubry (1983) did not mention this species in her study of the Paleogene deposits of Northwest Europe. Bukry (1973, 1975) listed it as part of the assemblages of upper Paleocene to lowest Eocene Zones CP6 to CP9a. In California, it is present in the Lodo Formation in Units 1 and 2, equivalent to upper Paleocene Zones CP6 to CP8, and sporadically in the Unit 3 equivalent of CP11, where it may be reworked (Bramlette and Sullivan, 1961). Müller (1974) reported it in upper Paleocene to lower Eocene Zones NP8 to NP12 (CP7-CP10) from Indian Ocean cores. The probable disappearance of $E$. distichus in CP10* (NP12*), if confirmed by further study, may serve as a useful datum.

Ellipsolithus lajollaensis, a species closely related to E. distichus, first occurs at Site 612 in Sample 612-50-2, 20-22 cm (455.41 m), in Zone CP11* (NP13*), and ranges through the lower Eocene. At Site 613 its first appearance, in Sample 613-43-4, 20-21 cm (491.80 m), near the first appearances of $C$. pseudogammation and $R$. tenuis, supports the interpretation that an unconformity is present in the section (compare species ranges at Sites 612 and 613; Figs. 4, 5). The overall range of this species has not been delineated by previous workers, possibly because of its relatively small size. Bukry and Percival (1971) described it and reported its occurrence in the lower middle Eocene, but its range was unknown. They listed it as occurring in the Donzacq Marl (Donzacq, France), which Hay and Mohler (1967) referred to the Discoaster lodoensis Zone (CP11; NP13). In a recent study, Aubry (1983) did not observe it in the many sections she investigated in the Paleogene of the AngloParis Basin. Bukry (1973) listed it in the assemblages of Subzones CP12a and CP12b that encompass the lower Eocene/middle Eocene boundary. Bybell and Gibson (1985) reported its first occurrence in the lower part of Zone NP14 (CP12a) in the Tallahatta Formation (Alabama and Georgia). However, Bukry et al. (1977) listed it in an assemblage (Mf2051) from an unnamed shale in the Santa Teresa Hills (California) that was assigned to Zone CP11. Further study is required to determine the range of $E$. lajollaensis in more detail. At present, the evidence available suggests that its first occurrence is in Zone CP11* (NP13*), below the first occurrence of Rhabdosphaera tenuis.

Ellipsolithus macellus last occurs at Site 612 in Sample $612-54-2,20-22 \mathrm{~cm}(494.21 \mathrm{~m})$, in the lower part of Zone CP11* (NP13*), not far above the first occurrences of $H$. lophota and $C$. crassus. It is present consistently in the section below this level. The same relationship is present at Site 613, where it last occurs in Sample 613$44-4,20-21 \mathrm{~cm}(501.11 \mathrm{~m})$. At both sites, E. macellus is 
present in Subzone CP9b (NP11), the oldest strata recovered. The reported first occurrence of $E$. macellus marks the base of upper Paleocene Zone NP4; it ranges into lower Eocene Zone NP12, and probably extends into the upper part of NP12 (Martini, 1971). Similarly, the first occurrence of the species marks the base of Zone CP3, and it was listed in assemblages of Zones CP5 to CP10 (Bukry, 1973; Okada and Bukry, 1980). In the Anglo-Paris Basin it occurs sporadically and is recorded only from Zones NP8 and NP11 (Aubry, 1983). In California, Bramlette and Sullivan (1961) reported it in Units 1, 2, and 3 of the Lodo Formation, the equivalent of Zones CP6, CP7, CP8, CP9b, CP10, and CP11, but not in the overlying Domengine Formation (Unit 4; CP12a). In the Tallahatta Formation of Alabama and Georgia, Bybell and Gibson (1985) recorded its last occurrence in the lower part of NP12 (CP10). The last occurrence of E. macellus at Sites 612 and 613 is above the first occurrences of $H$. lophota and $C$. crassus and below the last occurrence (unreworked) of $T$. orthostylus, and places this datum in the lower part of Zones CP11* and NP13*.

Rhabdosphaera inflata first appears at Site 612 in Sample 612-39-4, 20-22 cm (352.01 m), at the base of Subzone CP12*b* (NP14*b); and its last occurrence is in Sample $612-37-3,80 \mathrm{~cm}(331.90 \mathrm{~m})$, at the top of the same subzone. At Site 613, it first occurs in Sample 613$37-6,130 \mathrm{~cm}(439.30 \mathrm{~m})$. This distinctive species has a short stratigraphic range, alluded to by Bramlette and Sullivan (1961, p. 137), on the evidence of samples from the Canoas member of the Kreyenhagen Formation (California) and from Gibret in southwestern France. The range of $R$. inflata was documented within the $D$. sublodoensis Zone (CP12, NP14) in oceanic cores (Bukry, 1971, 1973), in Blake Plateau cores (Gartner, 1971), in the Aspe section of southeastern Spain (Romein, 1979), in the Anglo-Paris Basin (Aubry, 1983), and in the Tallahatta Formation of Alabama and Georgia (Bybell and Gibson, 1985). The $R$. inflata first-occurrence datum at the base of CP12b marks the lower Eocene/middle Eocene boundary (Bukry, 1973, 1981; Okada and Bukry, 1980; Berggren et al., 1985). The last occurrence of $R$. inflata and the first occurrence of Nannotetrina fulgens (= N. quadrata) were used by Bukry (1973) to delineate the CP12/CP13 (NP14/NP15) boundary, and the implication is that the two datums are practically coeval (Fig. 2). Martini (1971) employed only the first occurrence of $N$. fulgens $(=N$. alata $)$ to mark the NP14/NP15 boundary. Bramlette and Sullivan (1961) reported the two species to be present in a sample from the Canoas member of the Kreyenhagen Formation (California). Romein (1979) showed the last occurrence of $R$. inflata to be below, and separated by $10 \mathrm{~m}$ from, the first occurrence of $N$. fulgens in the Aspe section (southeastern Spain), but unfortunately he did not examine sediment within the interval. Aubry (1983) concluded that $R$. inflata occurred in the upper part of NP14 $(=\mathrm{CP} 12 \mathrm{~b})$ and the lowest part of NP15, thus overlapping the first occurrence of $N$. fulgens. In the present study, the top of Zone CP12* (NP14*) is placed at the last occurrence of $R$. inflata. The restricted stratigraphic range and wide geographic occurrence of this species make it suitable for use both as a first-occurrence and a last-occurrence datum. In addition, $R$. inflata is a species that is distinctive in polarized light and, from observation, may be more resistant to dissolution than $N$. fulgens, which has been tentatively identified at Sites 612 and 613 and may be represented by poorly preserved specimens. The choice of $R$. inflata instead of $N$. fulgens also avoids any uncertainty surrounding the taxonomy of $N$. fulgens, $N$. alata, and $N$. quadrata. The first occurrence of $N$. fulgens is retained as a secondary marker for the upper boundary of CP12* (NP14*), in the event $R$. inflata is absent.

Many of the species discussed in the foregoing are significant because their first or last occurrences can be utilized as primary or secondary datums in the zonation of lower Eocene strata; others show promise as marker species. There are also important elements in the nannofossil assemblages at Sites 612 and 613 that range through the lower Eocene studied here and elsewhere in the world, including such species as Campylosphaera dela, Cepakiella lumina, Coccolithus cribellum, C. magnicrassus, Cyclococcolithina gammation, and Discoasteroides kuepperi. Many of the other species present in the lower Eocene strata at Sites 612 and 613 at present are not useful in detailed biostratigraphic studies. Some species are difficult to identify because of morphologic variation, some are prone to dissolution, and first- and last-occurrence datums are sometimes hard to determine because evolutionary relationships are unclear. Species of the genera Chiasmolithus, Discoaster, and Reticulofenstra, among others, present problems in this regard. Tables 1 and 2 present the stratigraphic ranges at Sites 612 and 613 of selected species that meet the criteria set forth at the beginning of this chapter.

\section{ZONATION OF THE LOWER EOCENE}

\section{Justification for Revision}

We have seen that earlier studies of the lower Eocene from continental and oceanic areas have culminated in several zonation schemes for calcareous nannofossils that are similar in the choice of datums and in the delineation of zones and subzones (Hay, 1964, 1967; Hay and Mohler, 1967; Mohler and Hay, 1967; Martini, 1970, 1971; Bukry, 1973, 1975, 1978, 1981; Okada and Bukry, 1980; see Aubry, 1983, appendix 3 for a comparison of various Eocene zonations). A purpose of this study is to evaluate lower Eocene biozones that have been described by various authors and incorporated into the Martini NP and Bukry-Okada CP zonation schemes, to determine their similarities, and to decide whether it is advantageous to alter the lower Eocene zonation by revising those zones that are similar conceptually but that differ somewhat in their boundary datums. It is apparent that the biozones of the Martini and Bukry-Okada zonations have much in common and that many of them share a boundary datum (Fig. 2). The Discoaster binodosus Subzone (CP9b) and the $D$. binodosus Zone (NP11) are equivalent by definition, as is the Discoaster sublodoensis Zone (CP12; NP14) of both zonations. The following paired zones share the same formal name and the same upper 
boundary datum: CP9a and NP10; CP11 and NP13; CP12 and NP14. Likewise, CP10 and NP12 share the same formal name and lower boundary datum. The only differences between the two schemes are in the choice of boundary datums for the bases of CP9 and NP10, and for the boundaries between CP10 and CP11 and between NP12 and NP13.

Recent publications by Okada and Bukry (1980, table 1, not table 2) and Bukry (1981, fig. 4) indicate a correspondence between the following pairs of zones: CP9a and NP10; CP9b and NP11; CP10-CP11 and NP12NP13; CP12 and NP14. Moreover, Berggren, Kent, Flynn, and Van Couvering, 1985, fig. 4) have equated these zonal pairs as well as the remaining pairs, CP10 and NP12, CP11 and NP13. In effect, these studies suggest that all of the lower Eocene zones and most of the subzones of the Martini NP zonation have counterparts in the Bukry-Okada CP zonation.

The conclusions of this discussion and the preceding section on species occurrence suggest that revision of the Martini and Bukry-Okada lower Eocene biozones, with the purpose of equalizing and refining them, is justified on the following grounds:

1. Lower Eocene NP and CP biozones presently are either equivalent of differ little in the rock record they represent; and they share many of the same boundary criteria.

2. species whose range terminations are used as boundary datums, and species commonly present within the $\mathrm{NP}$ and $\mathrm{CP}$ zones, are present at both high-latitude and low-latitude localities.

3. Species whose range terminations are used here as boundary datums for newly erected subzones are easily recognizable and widely distributed in Europe, the Middle East, the United States, and the world's ocean basins.

4. At present, the $\mathrm{CP}$ zonation is more refined than the NP zonation, but the latter is more widely used. Revision improves communication among stratigraphers by removing ambiguities in NP and $\mathrm{CP}$ zonal correlation, by incorporating the strong points of the $\mathrm{CP}$ zonation, and by introducing new subzones that refine the biostratigraphy and potentially improve correlation, age-dating, the recognition of unconformities, and the calculation of sediment accumulation rates.

\section{Biostratigraphic Units-Formal Names and Informal Codes}

The biozones of the Martini and Bukry-Okada zonations, in addition to their formal names, also are identified by informal alphanumeric codes (e.g., NP12, CP10). These code names are informal labels for the biozones, and are highly useful as a shorthand notation for communication between workers in the many disciplines that utilize biostratigraphy as a basis for age-dating and correlation.

Procedures have been established by stratigraphic codes for erecting and revising formal biozones and their names (International Subcommission on Stratigraphic Classification, 1976; North American Commission on Stratigraphic Nomenclature, 1983). By contrast, alphanumer- ic code names of biozones inherently are not easy to revise, and therefore there is no provision for doing so in the North American Stratigraphic Code of 1983. Formal biozone names usually incorporate the name of one or two nannofossil taxa that occur within the biozone or that define its upper and/or lower boundaries. In practice, however, formal names (e.g., Discoaster lodoensis partial-range Zone) are cumbersome, and alphanumeric codes are employed for convenience.

A dilemma arises when a biozone is revised by the rules of stratigraphic nomenclature. Since the lower Eocene biozones that are revised herein are not modified substantially in concept, a new formal name is not required (North American Commission on Stratigraphic Nomenclature, 1983, Article 54, Section 2b). On the other hand, with regard to the informal code names, we must decide whether to (1) retain the existing code name for the zone and risk confusion among fellow workers; (2) establish a new code just for the lower Eocene that may not gain acceptance; or (3) compromise by altering minimally the existing Martini and Bukry-Okada NP and CP codes so that their overall utility can be improved while their familiarity to the scientific community is maintained. In this study I have chosen the third alternative. Lower Eocene biozones are formally revised and new subzones established, but the NP and CP codes of the Martini and Bukry-Okada zonations are retained. They are slightly modified in two ways; (1) by the addition of an asterisk $(*)$, a new practice that signals a revision in the original biozone or subzone definition; (2) by the addition of lowercase letters $(a, b, c)$ to identify, within the existing codes, new subzones defined here. For example, the codes NP14* and CP12* indicate that zones NP14 and CP12 have been revised from their original definitions; it follows that NP14*a is a new or unrevised subzone, and $\mathrm{CP} 12^{*} \mathrm{~b}^{*}$ is a revised subzone. By this method, lower Eocene Martini and Bukry-Okada biozones and subzones can be revised, while at the same time retaining the widely used alphanumeric codes that have made the biozonations useful to a broad audience.

In a study of DSDP Leg 73 sites in the South Atlantic, Percival (1984) delineated several Martini zones on the basis of secondary nannofossil datums (primary datums were not present), and he appended an asterisk $\left({ }^{*}\right)$ to the corresponding NP codes names. It is clear that Percival's recognition of NP zones on the basis of secondary datums applies only to DSDP Leg 73 sites, and this use does not constitute a formal revision of Martini's zones. Moreover, Percival listed the NP zones in terms of their original names and boundary datums, and explained the use of secondary datums at Leg 73 sites in remarks. Lower Eocene Zones NP10* and NP11* of Percival $(1984$, p. 392$)$ should not be confused with Zones NP10* and NP11* of the present study. The implications of Percival's biostratigraphy at Leg 73 sites for the age-dating of nannofossil datums have been discussed by Bukry (1985), Hsü (1985), and Hsü et al. (1984).

Although at present it is advisable to revise the biostratigraphic zonation without introducing wholly new alphanumeric codes, this is not a permanent solution to the problem. In the rapidly developing discipline of stra- 
tigraphy, a level of knowledge inevitably will be reached that warrants a new code not only incorporating the many revisions of the old one but also capable of accomodating future refinement.

\section{Revised Zonation of the Lower Eocene}

Calcareous nannofossils at Sites 612 and 613 are generally abundant and well preserved, and the extraordinary thickness of the lower Eocene strata here (198 m at Site 612 ) provided an opportunity to delineate accurately the range relationships of stratigraphically important species. These species are consistently present in the section within their previously recorded stratigraphic ranges, and only first occurrences are employed as datums for the new subzones established here. The present study revises biozones in three ways, as illustrated in Figure 3: (1) it formally emends boundary datums so that similarly named biozones of the CP and NP zonations now are based on the same boundary criteria (e.g., base of CP9 and NP10; boundary between CP10 and 11 and between NP 12 and 13); (2) it emends the boundary for the tops of Zones CP12 and NP14 by substituting a more reliable and distinctive marker as a datum; and (3) it erects two new subzones for CP10 and NP12, and three new subzones for CP11 and NP13.

\section{Discoaster diastypus interval Zone}

Informal code names. CP9; NP10*-11.

Definition. Interval from the first occurrence of Discoaster diastypus Bramlette and Sullivan, 1961, to the first occurrence of Discoaster lodoensis Bramlette and Riedel, 1954.

Age. Early Eocene.

Important event. The first occurrence of Tribrachiatus contortus is a secondary datum for the base of this zone.

Authors. Discoaster diastypus Zone Bukry, 1973; Bukry, 1975 (erected Tribrachiatus contortus Subzone and Discoaster binodosus Subzone); = CP9, Okada and Bukry, 1980.

Reference area. Oceanic strata (Deep Sea Drilling Project).

Remarks. The $D$. diastypus Zone is the oldest zone of the early Eocene. It was divided into two subzones by Bukry (1975). The present revision recognizes Zones NP10 and NP11 as subzones of the $D$. diastypus Zone. Subzone NP10*, as used here, is not the same as NP10* of Percival (1984). For selected species occurring in this zone, and for further explanation, see the subzone descriptions that follow.

\section{Tribrachiatus contortus interval Subzone}

Informal Code Names. CP9a; NP10*.

Definition. Interval from the first occurrence of Discoaster diastypus Bramlette and Sullivan, 1961, to the last occurrence of Tribrachiatus contortus (Stradner, 1958).

Age. Early Eocene.

Important event. The first occurrence of Tribrachiatus contortus is a secondary datum for the base of this subzone.

Authors. Marthasterites contortus Zone Hay, 1984; = NP10, Martini, 1970, 1971.

Tribrachiatus contortus Subzone (Hay, 1964) emend. Bukry, 1975; = CP9a, Okada and Bukry, 1980. 392).

Not Tribrachiatus contortus Zone, NP10* of Percival (1984, p.

Reference areas. Switzerland; oceanic strata (deep Sea Drilling Project).

Selected species (from the literature). Campylosphaera dela, Chiasmolithus consuetus, Discoaster binodosus, $D$. diastypus, $D$. lenticularis, D. multiradiatus, D. nobilis, Ellipsolithus distichus, E. macellus, Lopodolithus nascens, Sphenolithus radians, Toweius craticulus, Tribrachiatus contortus, T. nunnii, T. orthostylus.

Remarks. Subzone CP9a is equivalent to Zone NP10 (Martini, 1971), according to Okada and Bukry (1980, table 1), Bukry (1981, fig. 4), and Berggren et al. (1985, fig. 4). Both units are delineated at the top by the last occurrence of $T$. contortus. The basal boundary for the Tribrachiatus contortus Zone (NP10) was the first occurrence of $T$. nunnii ( = new name substituted for Marthasterites bramlettei by Gartner, 1971, p. 116; Bukry, 1975), but it was emended by Bukry (1975), to be the first occurrence of $D$. diastypus. This zone, now a subzone, is designated NP10* in recognition of Bukry's revision of the basal datum, which made it coeval with Subzone CP9a. Bukry (1975) included the first occurrence of $T$. contortus as a secondary datum for the base of CP9a, although it was not so stated in the text description of the subzone. Tribrachiatus nunnii evolves into $T$. contortus near the base of this subzone (Mohler and Hay, 1967; Martini, 1971). In addition, T. nunnii, $D$. diastypus, and $T$. contortus first occur, in that order, within 3 to $4 \mathrm{~m}$ in the Nahal Avdat section in Israel; similarly, the first occurrences of $T$. nunnii and $D$. diastypus are separated by 1 to $2 \mathrm{~m}$ in the Caravaca section in southeastern Spain (Romein, 1979). NP10*, as used here, should not be confused with NP10* of Percival (1984, p. 392), a local variation of NP10 used solely at DSDP Leg 73 sites (South Atlantic) where the primary datums of the zone are absent.

\section{Discoaster binodosus partial-range Subzone}

Informal code names. CP9b; NP11.

Definition. Interval from the last occurrence of Tribrachiatus contortus (Stradner, 1958) to the first occurrence of Discoaster lodoensis Bramlette and Riedel, 1954.

Age. Early Eocene.

Authors. Discoaster binodosus Zone Mohler and Hay, 1967; = NP11, Martini, 1970, 1971; D. binodosus Subzone Bukry, 1975; = CP9b, Okada and Bukry, 1980.

Reference areas. Switzerland; oceanic strata (Deep Sea Drilling Project)

Selected species (from the literature and this study). Campylosphaera dela, Chiasmolithus californicus, C. consuetus, C. grandis, Coccolithus magnicrassus, Discoaster barbadiensis, D. binodosus, $D$. diastypus, D. lenticularis, D. nobilis, Ellipsolithus macellus, Lophodolithus nascens, Sphenolithus radians, Tribrachiatus orthostylus.

Remarks. The Discoaster binodosus (sub)Zone of Mohler and Hay (1967) became a part of the Martini $(1970,1971)$ zonation, and later was incorporated into the Bukry (1975) zonation; NP11 and CP9b are equivalent by definition.

\section{Tribrachiatus orthostylus partial-range Zone (emended)}

Informal code names. CP10*; NP12*.

Definition. Interval from the first occurrence of Discoaster lodoensis Bramlette and Riedel, 1954, to the first occurrence of Helicosphaera lophota (Bramlette and Sullivan, 1961).

Age. Early Eocene.

Important event. The first occurrence of Coccolithus crassus is a secondary datum for the top of this zone.

Authors. Marthasterites tribrachiatus Zone Brönnimann and Stradner, 1960; Brönnimann and Rigassi, 1963; = NP12, Martini, 1970, 1971.

Tribrachiatus orthostylus Zone (Brönnimann and Stradner, 1960) emend. Bukry, 1973; = CP10, Okada and Bukry, 1980.

Tribrachiatus orthostylus Zone (Bukry, 1973) emend. Valentine, this paper.

Reference areas. Cuba; oceanic strata (Deep Sea Drilling Project); New Jersey continental slope and rise, DSDP Sites 612 and 613 .

Remarks. This emendation makes the $T$. orthostylus Zone NP12* equivalent to the $T$. orthostylus Zone CP10*. Brönnimann and Stradner (1960) defined the $T$. orthostylus Zone as the interval from the first occurrence of Discoaster lodoensis to the last occurrence of $T$. orthostylus, with the reference area in Cuba (Brönnimann and Rigassi, 1963; Hay and Mohler, 1967; Mohler and Hay, 1967); and Martini $(1970,1971)$ gave it the alphanumeric designation NP12. Subsequently, Bukry (1973) revised the $T$. orthostylus Zone to be the interval from the first occurrence of $D$. lodoensis to the first occurrence of Coccolithus crassus, a biozone encompassing somewhat less time than NP12, because the first occurrence of C. crassus is stratigraphically below the last occurrence of $T$. orthostylus (Bukry, 1978, fig. 12). Okada and Bukry (1980) designated this Zone CP10. Although Bukry (1973) in effect emended the T. orthostylus Zone, both definitions are in use. Berggren et al. (1985) showed CP10 and NP12 to be coeval. 
Bukry $(1973,1978)$ chose the first occurrence of Coccolithus crassus to replace the last occurrence of $T$. orthostylus as a datum for the top of this zone, because $T$. orthostylus had been observed ranging into zones younger than NP12, and also because it is good practice to use first occurrences where possible, in order to reduce the likelihood of a spurious last occurrence by reworking upward in the section. $T$. orthostylus was recorded in the middle Eocene of California and at DSDP Site 245 in the Indian Ocean (Bukry, 1978), and a search of recorded ranges reveals its presence in strata assigned to younger zones (Perch-Nielsen, 1977, pp. 706, 721; Okada and Thierstein, 1979, tables 5B, 6A). T. orthostylus is present in samples studied here that lie well above its range, as determined by its persistent occurrence in a series of closely spaced samples (Figs. 4, 5). At Site 612, one specimen of $T$. orthostylus was observed at a depth of $434.24 \mathrm{~m}$, within the new Cyclicargolithus pseudogammation Subzone (CP11*b; NP13*b) and similarly at Site 613 at $440.91 \mathrm{~m}$, within the Discoasteroides kuepperi Subzone (CP12*a; NP14*a). Thus, the last occurrence of $T$. orthostylus is of questionable use as a datum. The first occurrence of $C$. crassus is a better choice to mark the top of the zone, but its range has not been well documented in published records, and it does not seem to have been accepted widely for use as a datum. The first occurrence of C. crassus was shown by Bukry $(1978$, fig. 12) to be below the last occurrence of $T$. orthostylus. The present study at DSDP Sites 612 and 613 has shown that the first occurrence of $H$. lophota is at the same level as the first occurrence of $C$. crassus and below the last occurrence (unreworked) of $T$. orthostylus. In addition, the first occurrence of $H$. lophota was recorded below the last occurrence of $T$. orthostylus in the Lodo Formation of California (Bramlette and Sullivan, 1961; Warren, 1983 ) and in the Tallahatta Formation of Alabama and Georgia (Bybell and Gibson, 1985). A comparison of the ranges of $C$. crassus, $H$. lophota, and $H$. seminulum as reported in two studies of the same sections of the Lodo Formation, shows that the first occurrences of $H$. lophota and $C$. crassus are practically coincident, and that the first occurrence of $H$. seminulum is somewhat below these two datums (Bramlette and Sullivan, 1961, table 1; Warren, 1983, table 3; Berggren and Aubert, 1983, fig. 4). The same relationship is present at DSDP Sites 612 and 613. These observations suggest that the first occurrences of $H$. lophota and $C$. crassus almost coincide, but it is unlikely that they are actually coeval. Helicosphaera lophota is a distinctive species, more widely recognized and recorded in biostratigraphic studies than $C$. crassus, and recently Hazel et al. (1984) have noted the importance of its first occurrence as a datum and employed it informally to mark the boundary between the $T$. orthostylus and $D$. lodoensis zones. Therefore, the first occurrence of $H$. lophota is selected here as the primary datum defining the top of the $T$. orthostylus Zone (CP10*; NP12*); the first occurrence of C. crassus is of secondary importance. The $T$. orthostylus Zone is subdivided into two subzones herein.

\section{Lophodolithus nascens partial-range Subzone (new subzone)}

Informal code names. CP10*a; NP12*a.

Definition. Interval from the first occurrence of Discoaster lodoensis Bramlette and Riedel, 1954, to the first occurrence of Helicosphaera seminulum Bramlette and Sullivan, 1961.

Age. Early Eocene.

Author. Valentine, this paper.

Reference area. New Jersey continental slope and rise, DSDP Sites 612 and 613 .

Selected species. Campylosphaera dela, Chiasmolithus californicus, C. consuetus, C. grandis, Coccolithus cribellum, C. magnicrassus, Discoaster diastypus, D. lodoensis, Discoasteroides kuepperi, Ellipsolithus distichus, E. macellus, Lophodolithus nascens, Sphenolithus radians, Tribrachiatus orthostylus.

Remarks. The Lophodolithus nascens Subzone (CP10*a; NP12*a) is a new subzone that encompasses the lower part of the emended Tribrachiatus orthostylus Zone (CP10*; NP12*), which is divided into two parts in this study.

Helicosphaera seminulum interval Subzone (new subzone)

Informal code names. CP10*b; NP12*b.

Definition. Interval from the first occurrence of Helicosphaera seminulum Bramlette and Sullivan, 1961, to the first occurrence of Helicosphaera lophota (Bramlette and Sullivan, 1961).
Age. Early Eocene.

Important events. The first occurrence of Coccolithus crassus is a secondary datum for the top of this subzone.

Chiasmolithus californicus is absent or occurs sporadically within this subzone.

Ellipsolithus distichus probably has its last occurrence within this subzone.

Lophodolithus nascens probably has its last occurrence near the top of this subzone or near the base of the succeeding subzone (CP11*a; NP13*a).

Reticulofenestra has its first occurrence within this subzone; represented by small, undescribed species.

Author. Valentine, this paper.

Reference area. New Jersey continental slope and rise, DSDP Sites 612 and 613 .

Selected species. Campylosphaera dela, Cepekiella lumina, Chiasmolithus consuetus, C. grandis, Coccolithus cribellum, C. magnicrassus, Cyclococcolithina formosa, C. gammation, Discoaster diastypus, D. lodoensis, Discoasteroides kuepperi, Ellipsolithus distichus (probable last occurrence), E. macellus, Helicosphaera seminulum, Lophodolithus nascens (probable last occurrence), Reticulofenestra genus (first occurrence), Sphenolithus radians, Tribrachiatus orthostylus.

Remarks. The Helicosphaera seminulum Subzone (CP10*b; NP12*b) is a new subzone that encompasses the upper part of emended Zone CP10*, T. orthostylus Zone (Bukry, 1973; Okada and Bukry, 1980 ), and the upper part of emended Zone NP12*, T. orthostylus Zone (Martini, 1971). (See remarks in the section describing and emending the $T$. orthostylus Zone, this chapter). The upper boundary of the $H$. seminulum Subzone is drawn at the first occurrence of $H$. lophota, which coincides at Sites 612 and 613 with the first occurrence of $C$. crassus. Further work is required to determine if this range relationship between $H$. lophota and $C$. crassus is valid. The first occurrence of the genus Reticulofenestra is an important event within this subzone. The early forms of the genus are small, apparently undescribed, and may represent several species. It would be difficult to determine the upper and lower limits of each of these small forms, as it is even for the larger described species, such as $R$. coenura and $R$. dictyoda.

\section{Discoaster lodoensis partial-range Zone (emended)}

Informal code names. CP11*; NP13*.

Definition. Interval from the first occurrence of Helicosphaera lophota (Bramlette and Sullivan, 1961) to the first occurrence of Discoaster sublodoensis Bramlette and Sullivan, 1961.

Age. Early Eocene.

Important event. The first occurrence of Coccolithus crassus is a secondary datum for the base of this zone.

Authors. Discoaster lodoensis Zone Brönnimann and Stradner, 1960; Brönnimann and Rigassi, 1963; = NP13, Martini, 1970, 1971.

Discoaster lodoensis Zone (Brönnimann and Stradner, 1960) emend. Bukry, 1973; = CP11, Okada and Bukry, 1980.

Discoaster lodoensis Zone (Bukry, 1973) emend. Valentine, this paper.

Reference areas. Cuba; oceanic strata (Deep Sea Drilling Project); New Jersey continental slope and rise, DSDP Sites 612 and 613.

Remarks. This zone is emended by selecting the first occurrence of $H$. lophota to replace the first occurrence of Coccolithus crassus and the last occurrence of Tribrachiatus orthostylus as a datum for the base of the zone. Revised Zones CP11* and NP13* now represent the same stratigraphic interval. Berggren et al. (1985b) showed CP11 and NP13 to be coeval, although the bases of the zones are defined by different datums. See explanation in preceding section describing and emending the Tribrachiatus orthostylus zone.

\section{Helicosphaera lophota interval Subzone (new subzone)}

Informal code names. $\mathrm{CP} 11^{*} \mathrm{a}$; $\mathrm{CP} 13^{*} \mathrm{a}$.

Definition. Interval from the first occurrence of Helicosphaera lophota (Bramlette and Sullivan, 1961) to the first occurrence of $C y$ clicargolithus pseudogammation (Bouche, 1962).

Age. Early Eocene.

Important events. The first occurrence of Coccolithus crassus is a secondary datum for the base of this subzone.

Ellipsolithus macellus has its last occurrence within this subzone. 
Tribrachiatus orthostylus has its last occurrence within this subzone.

Author. Valentine, this paper.

Reference area. New Jersey continental slope and rise, DSDP Sites 612 and 613.

Selected species. Campylosphaera dela, Cepekiella lumina, Chiasmolithus consuetus, $C$. grandis, Coccolithus crassus, $C$. cribellum, $C$. magnicrassus, Cyclococcolithina formosa, C. gammation, Discoaster diastypus, D. lodoensis, Discoasteroides kuepperi, Ellipsolithus macellus (last occurrence), Helicosphaera lophota, H. seminulum, Sphenolithus radians, Tribrachiatus orthostylus (last occurrence).

Remarks. The Helicosphaera lophota Subzone (CP11*a; NP13*a) is a new subzone that encompasses the lower part of the emended $D$. lodoensis Zone (CP11*; NP13*), which is divided into three parts in this study.

\section{Cyclicargolithus pseudogammation interval Subzone (new subzone)}

Informal code names. CP11*b; NP13*b.

Definition. Interval from the first occurrence of Cyclicargolithus pseudogammation (Bouche, 1962) to the first occurrence of Rhabdosphaera tenuis Bramlette and Sullivan, 1961.

Age. Early Eocene.

Important events. Ellipsolithus lajollaensis has its first occurrence within this subzone.

Lophodolithus mochlophorus probably has its first occurrence within this subzone.

Author. Valentine, this paper.

Reference area. New Jersey continental slope and rise, DSDP Sites 612 and 613.

Selected species. Campylosphaera dela, Cepekiella lumina, Chiasmolithus consuetus, $C$. grandis, Coccolithus crassus, C. cribellum, C. magnicrassus, Cyclicargolithus pseudogammation, Cyclococcolithina formosa, C. gammation, Discoaster diastypus, D. lodoensis, Discoasteroides kuepperi, Ellipsolithus lajollaensis (first occurrence), Helicosphaera lophota, $H$. seminulum, Lophodolithus mochlophorus (probable first occurrence), Sphenolithus radians.

Remarks. The Cyclicargolithus pseudogammation Subzone $\left(\mathrm{CP} 11^{*} \mathrm{~b} ; \mathrm{NP} 13^{*} \mathrm{~b}\right)$ is a new subzone that encompasses the middle part of the emended D. lodoensis Zone (CP11*; NP13*), which is divided into three parts in this study.

\section{Rhabdosphaera tenuis interval Subzone (new subzone)}

Informal code names. $\mathrm{CP} 11^{*} \mathrm{c} ; \mathrm{NP} 13^{*} \mathrm{c}$.

Definition. Interval from the first occurrence of Rhabdosphaera tenuis Bramlette and Sullivan, 1961, to the first occurrence of Discoaster sublodoensis Bramlette and Sullivan, 1961.

Age. Early Eocene.

Author. Valentine, this paper.

Reference area. New Jersey continental slope and rise, DSDP Sites 612 and 613 .

Selected species. Campylosphaera dela, Cepekiella lumina, Chiasmolithus consuetus, $C$. grandis, Coccolithus crassus, C. cribellum, C. magnicrassus, Cyclicargolithus pseudogammation, Cyclococcolithina formosa, C. gammation, Discoaster lodoensis, Discoasteroides kuepperi, Ellipsolithus lajollaensis, Helicosphaera lophota, $H$. seminulum, Lophodolithus mochlophorus, Rhabdosphaera tenuis, Sphenolithus radians.

Remarks. The Rhabdosphaera tenuis Subzone $\left(\mathrm{CP} 11^{*} \mathrm{c}\right.$; NP13*c) is a new subzone that encompasses the upper part of the emended $D$. lodoensis Zone (CP11*; NP13*), which is divided into three parts in this study.

\section{Discoaster sublodoensis interval Zone (emended)}

Informal code names. CP12*; NP14*.

Definition. Interval from the first occurrence of Discoaster sublodoensis Bramlette and Sullivan, 1961, to the last occurrence of Rhabdosphaera inflata Bramlette and Sullivan, 1961.

Age. Early Eocene-middle Eocene.

Important event. The first occurrence of Nannotetrina fulgens is a secondary datum for the top of this zone.

Authors. Discoaster sublodoensis Zone Hay, 1964; = NP14, Martini, 1970, 1971; Bukry, 1973 (erected Discoasteroides kuepperi Subzone and Rhabdosphaera inflata Subzone); = CP12, Okada and Bukry, 1980.
Discoaster sublodoensis Zone (Hay, 1964) emend. Valentine, this paper.

Reference areas. California; oceanic strata (Deep Sea Drilling Project); New Jersey continental slope and rise, DSDP Sites 612 and 613.

Remarks. The D. sublodoensis Zones CP12 and NP14 are equivalent by definition. This emendation replaces the first occurrence of Nannotetrina fulgens $(=N$. alata, $N$. quadrata $)$ with the last occurrence of $R$. inflata as the datum for the upper boundary of the revised Discoaster sublodoensis Zone (CP12*; NP14*). Rhabdosphaera inflata is widespread geographically, restricted stratigraphically, distinctive in polarized light, and is possibly more resistant to dissolution than $N$. fulgens. The last occurrence of $R$. inflata and the first occurrence of $N$. fulgens are approximately coeval, and the $N$. fulgens datum is an appropriate secondary marker in the absence of $R$. inflata. The $D$. sublodoensis Zone (CP12*; NP14*) straddles the lower Eocene/middle Eocene boundary; the $D$. kuepperi Subzone lies within the lower Eocene and the $R$. inflata Subzone lies within the middle Eocene (Bukry, 1973, 1981; Okada and Bukry, 1980; Berggren, Kent, Flynn, and Van Couvering, 1985).

\section{Discoasteroides kuepperi partial-range Subzone}

Informal code names. CP12*a; NP14*a.

Definition. Interval from the first occurrence of Discoaster sublodoensis Bramlette and Sullivan, 1961, to the first occurrence of Rhabdosphaera inflata Bramlette and Sullivan, 1961.

Age. Early Eocene.

Authors. Discoasteroides kuepperi Subzone Bukry, 1973; = CP12a, Okada and Bukry, 1980.

Reference area. Oceanic strata (Deep Sea Drilling Project).

Selected species. Campylosphaera dela, Cepekiella lumina, Chiasmolithus consuetus, $C$. grandis, $C$. solitus, Coccolithus crassus, $C$. cribellum, C. magnicrassus, Cyclicargolithus pseudogammation, Cyclococcolithina formosa, C. gammation, Discoaster barbadiensis, $D$. lodoensis, D. sublodoensis, Discoasteroides kuepperi, Ellipsolithus lajollaensis, Helicosphaera lophota, H. seminulum, Lophodolithus mochlophorus, Rhabdosphaera tenuis, Sphenolithus radians.

Remarks. The D. kuepperi Subzone $\left(\mathrm{CP}_{12} * \mathrm{a}\right.$; NP14*a) is the lower part of the D. sublodoensis Zone (CP12*; NP14*), and is the youngest biozone of the lower Eocene. Several species are present sporadically in the subzone at Sites 612 and 613, including Coccolithus crassus, C. cribellum, Discoasteroides kuepperi, and Lophodolithus mochlophorus, and their last occurrences are not well documented (Figs. 4, 5; Tables 1, 2). Bukry (1973) listed all four species in the assemblages for the equivalent of Subzone CP12*a, but not for CP12*b*. Coccolithus crassus was last observed by Bramlette and Sullivan (1961, p. 135) in Unit 4 (Domengine Formation, California), the equivalent of Subzone CP12*a. Coccolithus cribellum ranges into the equivalent of CP13 (NP15) in the Aspe section in southeastern Spain (Romein, 1979). Discoasteroides kuepperi is present no higher than Subzone CP12*a at the Nahal Avdat section (Israel), but ranges into Subzone CP12*b* in the Aspe section (southeastern Spain) (Romein, 1979) and in the Tallahatta Formation (Alabama and Georgia) (Bybell and Gibson, 1985). Lophodolithus mochlophorus is last observed in Subzone CP12*a in the Aspe section (Romein, 1979).

\section{Rhabdosphaera inflata taxon-range Subzone (emended)}

Informal code names. CP12*b*; NP14*b.

Definition. Interval from the first occurrence of Rhabdosphaera inflata Bramlette and Sullivan, 1961, to the last occurrence of Rhabdosphaera inflata Bramlette and Sullivan, 1961.

Age. Middle Eocene.

Important event. The first occurrence of Nannotetrina fulgens is a secondary datum for the top of this subzone.

Authors. Rhabdosphaera inflata Subzone Bukry, 1973; = CP12b, Okada and Bukry, 1980.

Rhabdosphaera inflata Subzone (Bukry, 1973) emend, Valentine, this paper.

Reference areas. Oceanic strata (Deep Sea Drilling Project); New Jersey continental slope and rise, DSDP Sites 612 and 613.

Selected species (from the literature and this study). Campylosphaera dela, Cepekiella lumina, Chiasmolithus grandis, C. solitus, Coccolithus staurion, Cyclicargolithus pseudogammation, Cyclococcolithina formosa, Discoaster barbadiensis, D. lodoensis, D. mirus, D. 
strictus, D. sublodoensis, Ellipsolithus lajollaensis, Lophodolithus mochlophorus, Helicosphaera lophota, $H$. seminulum, $N$. fulgens (possibly near top of subzone), Rhabdosphaera inflata, $R$. tenuis, Sphenolithus radians.

Remarks. The $R$. inflata Subzone (CP12*b*; NP14*b) is the upper part of the D. sublodoensis Zone (CP12*; NP14*), and is the oldest subzone of the middle Eocene. See remarks on ranges of several sporadically occurring species in the preceding section on the $D$. kuepperi Subzone. This emendation replaces the first occurrence of Nannotetrina fulgens $(=N$. alata, $N$. quadrata $)$ with the last occurrence of $R$. inflata to mark the upper boundary of the subzone. See explanation in section describing and emending the Discoaster sublodoensis zone.

\section{CHRONOSTRATIGRAPHY OF LOWER EOCENE BIOZONES}

A refined biostratigraphic zonation provides a framework for correlating strata and for identifying unconformities within the rock record. If the ages of biostratigraphic datums and the duration of biozones can be established by direct radiometric dating or by correlation with radiometrically dated magnetic polarity stratigraphy, then among other things the accumulation rates of sedimentary sections can be determined. It follows thatwithin a section that contains a few dated events or datums, no major unconformities, and a constant or smoothly varying sediment accumulation rate-the ages of undated events can be approximated by plotting the depth of the datum in the section against age along the accumulation rate (or time-depth) curve. The present study has identified several biostratigraphic events in the lower Eocene that have been dated previously. The purpose here is to utilize them and the time-depth curve for Site 612 to obtain a preliminary estimate of the ages of nannofossil events that are selected here to define new subzones and that have not been dated by magnetostratigraphic methods.

The Paleogene time scale of Berggren, Kent, and Flynn (1985) and Berggren, Kent, Flynn, and Van Couvering (1985), drawing on data from numerous sources, is based on the radiometric dating of both biologic events (biochronology) and magnetic polarity-reversal stratigraphy (magnetochronology). Those authors (and references therein) give a full treatment of this topic and the results of the most recent studies. The practical application of bio- and magnetochronology is to determine the ages of stratigraphic datums, such as epoch and biozone boundaries, by correlating them with the magnetic reversal record contained in the strata. This is a very active field of research; ages determined by this method are approximations and are undergoing revision as more complete bio- and magnetostratigraphic records are discovered. At present, the ages of epoch and biozone boundaries (and their associated fossil datums) vary somewhat from study to study, and the choice of any one age depends on an assessment of the quality of the rock record and of the analytical methods upon which the study was based.

\section{Ages of Nannofossil Datums; Sediment Accumulation Rates}

The ages of several of the key lower Eocene nannofossil datums used here resulted from a study of DSDP Leg 74 cores (taken in the South Atlantic) (Shackleton,
1984) that employed the new magnetic-anomaly time scale of Berggren et al. (1985). At Sites 527 and 528, the percent of core recovery and the position of magnetic anomalies in relation to the recovered section suggest that the identification of Anomalies 21, 22, and 23 are correct. Within this interval, the following nannofossil datums and their ages were determined (Shackleton, 1984): the first occurrences of Nannotetrina fulgens $(=N$. quadrata) at 49.5 Ma and Discoaster sublodoensis at 51.7 $\mathrm{Ma}$; and the last occurrence of Tribrachiatus orthostylus at 54.0 Ma. Below Anomaly 23, and especially in the reversed interval between Anomalies 23 and 24, the dating of events is less certain, owing to changes in sedimentation rate, to low magnetic inclinations, and to lack of data. Only one age from this interval is utilized directly here, the first-occurrence datum of Discoaster lodoensis at $55.4 \mathrm{Ma}$, which appears to be near the base of Anomaly $24 \mathrm{a}$ at Site 527 .

Except for the D. sublodoensis datum, the ages of the nannofossil datums of Shackleton (1984) are substantially the same as those selected by Berggren, Kent, and Flynn (1985), who incorporated data from several sources, including DSDP Leg 74, into their study. Those authors estimate the first-occurrence datum of $D$. sublodoensis to be $52.6 \mathrm{Ma}$, on the basis of data from a section near Gubbio, Italy, provided to them by Monechi and Thierstein (in press). I have chosen to use the age of $51.7 \mathrm{Ma}$ for this datum on the basis of the apparently good stratigraphic control in the study by Shackleton (1984). However, there are many possible sources of error inherent in stratigraphic studies of this nature, and the correct identification of both the magnetic reversal boundaries and the nannofossil datums are critical to the results.

A plot of age against depth for the lower Eocene and lowest middle Eocene (CP10*-12*; NP12*-14*) at Site 612 -utilizing the ages of first occurrences of $N$. fulgens, $D$. sublodoensis, and D. lodoensis and the last occurrence (unreworked) of $T$. orthostylus - reveals the sediment accumulation rate for approximately $216 \mathrm{~m}$ of section (Fig. 6). Nannotetrina fulgens was not identified positively at Sites 612 and 613 , but at Site 612 fragments interpreted to be detached arms of the species are present in six samples from 329.81 to $332.81 \mathrm{~m}$, overlapping the last occurrence of $R$. inflata at $331.90 \mathrm{~m}$. The range of $R$. inflata is known at Site 612 , and because its last occurrence is approximately coeval with the first occurrence of $N$. fulgens (Bukry, 1973, 1978), the age of the $N$. fulgens FAD (49.5 Ma) is employed to approximate the age of the $R$. inflata LAD. The four datum points form three line segments that decrease in slope upward. The lower segment (D. lodoensis to $T$. orthostylus) represents a sediment accumulation rate of $4.9 \mathrm{~cm} / 10^{3} \mathrm{yr}$., the middle segment ( $T$. orthostylus to $D$. sublodoensis) a rate of $3.7 \mathrm{~cm} / 10^{3} \mathrm{yr}$., and the upper segment (D. sublodoensis to $R$. inflata) a rate of $2.8 \mathrm{~cm} / 10^{3}$ yr. Given a constant sediment accumulation rate between each set of points, the first occurrences of the other important species used to zone the lower Eocene at this site can be projected onto the lines to give an approximation of their ages.

Following this procedure with the lower line segment, we see that the age of the first occurrence of Helico- 


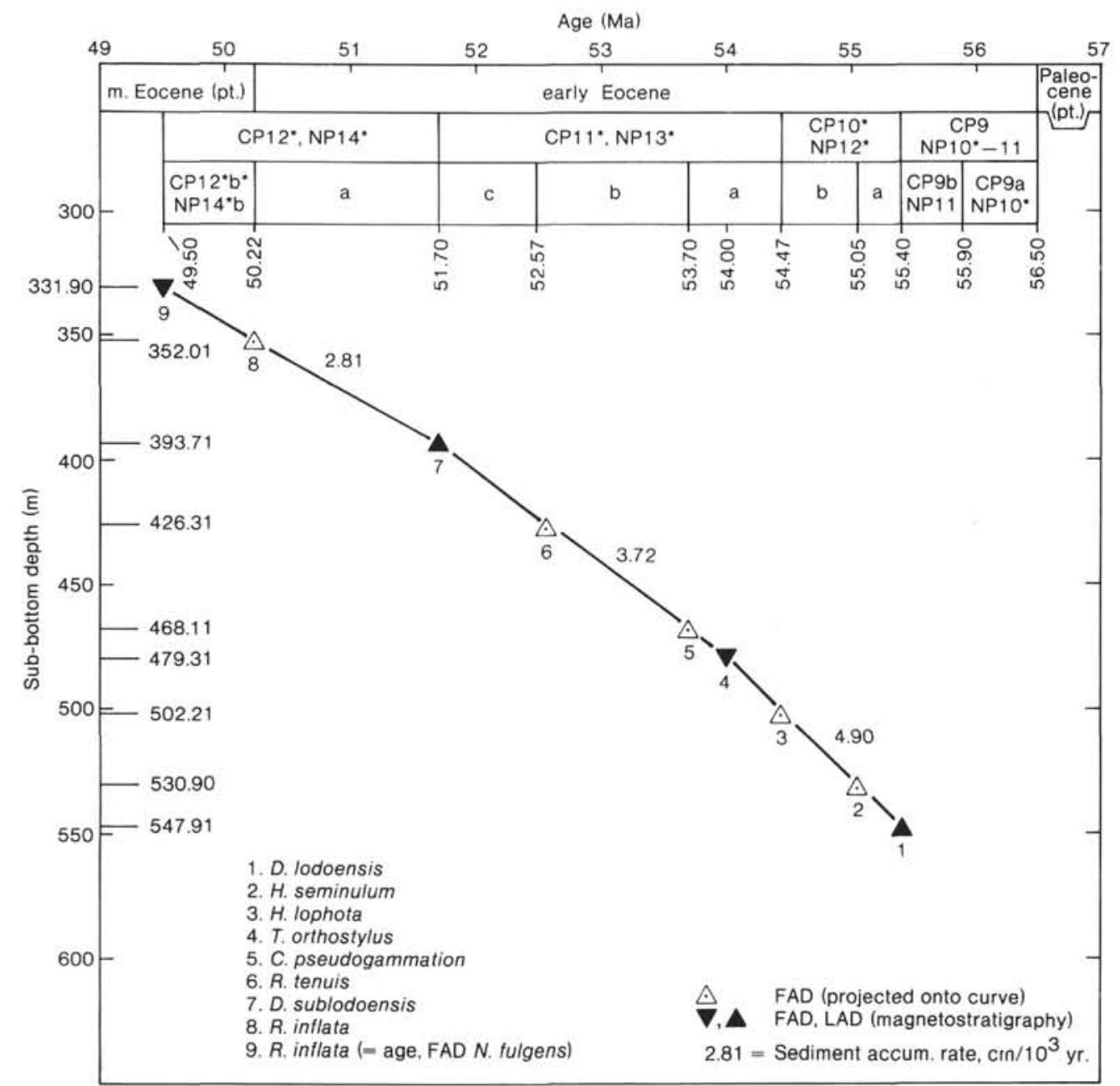

Figure 6. Time-depth plot of nannofossil datums at Site 612, New Jersey slope. Asterisk (*) superscripts on CP and NP codes indicate revision of original zone or subzone. FAD, first-appearance datum; LAD, last-appearance datum. Ages of datums, based on magnetostratigraphy (closed triangles; Shackleton, 1984), are used to constrain the curve. LAD of T. orthostylus is last unreworked occurrence. Ages of datums determined in this study (open triangles) are based on projection of FADs onto the curve. Sediment accumulation rate declines from $4.90 \mathrm{~cm} / 10^{3}$ yr. to $2.81 \mathrm{~cm} / 10^{3}$ yr. over a period of 5.90 m.y. There are no obvious hiatuses in this section, which is treated here as a standard for the western North Atlantic margin. Ages of datums shown here are used to construct time-depth plot for Site 613, New Jersey rise (Fig. 7).

sphaera seminulum is approximately $55.0 \mathrm{Ma}$ and that of $H$. lophota $54.5 \mathrm{Ma}$. Continuing to the middle and upper line segments, the first occurrences of Cyclicargolithus pseudogammation, Rhabdosphaera tenuis, and Rhabdosphaera inflata are approximately $53.7 \mathrm{Ma}, 52.6$ $\mathrm{Ma}$, and $50.2 \mathrm{Ma}$, respectively. An alternative solution for the middle interval, using the ages for the last occurrence of $T$. orthostylus $(53.7 \mathrm{Ma})$ and the first occurrence of $D$. sublodoensis $(52.6 \mathrm{Ma})$ given by Berggren, Kent, and Flynn (1985), would result in a sediment accumulation rate of $7.8 \mathrm{~cm} / 10^{3} \mathrm{yr}$., more than twice that favored here, and would alter considerably the ages of the $C$. pseudogammation and $R$. tenuis first-occurrence datums.

A time-depth plot of nannofossil datums at Site 613 on the upper rise, in part based on ages determined from the Site 612 curve, reveals a curve that is considerably different from the one at Site 612 on the middle slope (Fig. 7). The upper parts of the two curves, above the $D$. sublodoensis datum, are comparable. Major differences, however, are the presence of an unconformity at Site 613 that represents at least Subzone CP11*b (NP13*b), about $1.1 \mathrm{~m} . \mathrm{y}$. and possibly longer (Fig. 5), and a sediment accumulation rate $\left(9.7 \mathrm{~cm} / 10^{3} \mathrm{yr}\right.$.) in the lower part of the section at Site 613 that is twice that at Site 612. Each of these anomalies may be due to the effects of mass wasting, representing an accumulation of contemporaneously eroded sediment during Subzone CP10*a time and, later, the removal of sediment during at least Subzone CP11*b time. Sediment accumulation rates between the $H$. lophota and C. pseudogammation datums $(1.56 \mathrm{~cm} /$ $10^{3} \mathrm{yr}$.) and between the $R$. tenuis and $D$. sublodoensis datums $\left(2.40 \mathrm{~cm} / 10^{3} \mathrm{yr}\right.$.) are minimums, because the duration of the hiatus is not precisely known and probably is longer than $1.1 \mathrm{~m} . \mathrm{y}$.

In the absence of data extrapolated from Site 612, the biostratigraphic analysis of Site 613 would not have shown that strata representing Subzone CP11*b (NP13*b) are 


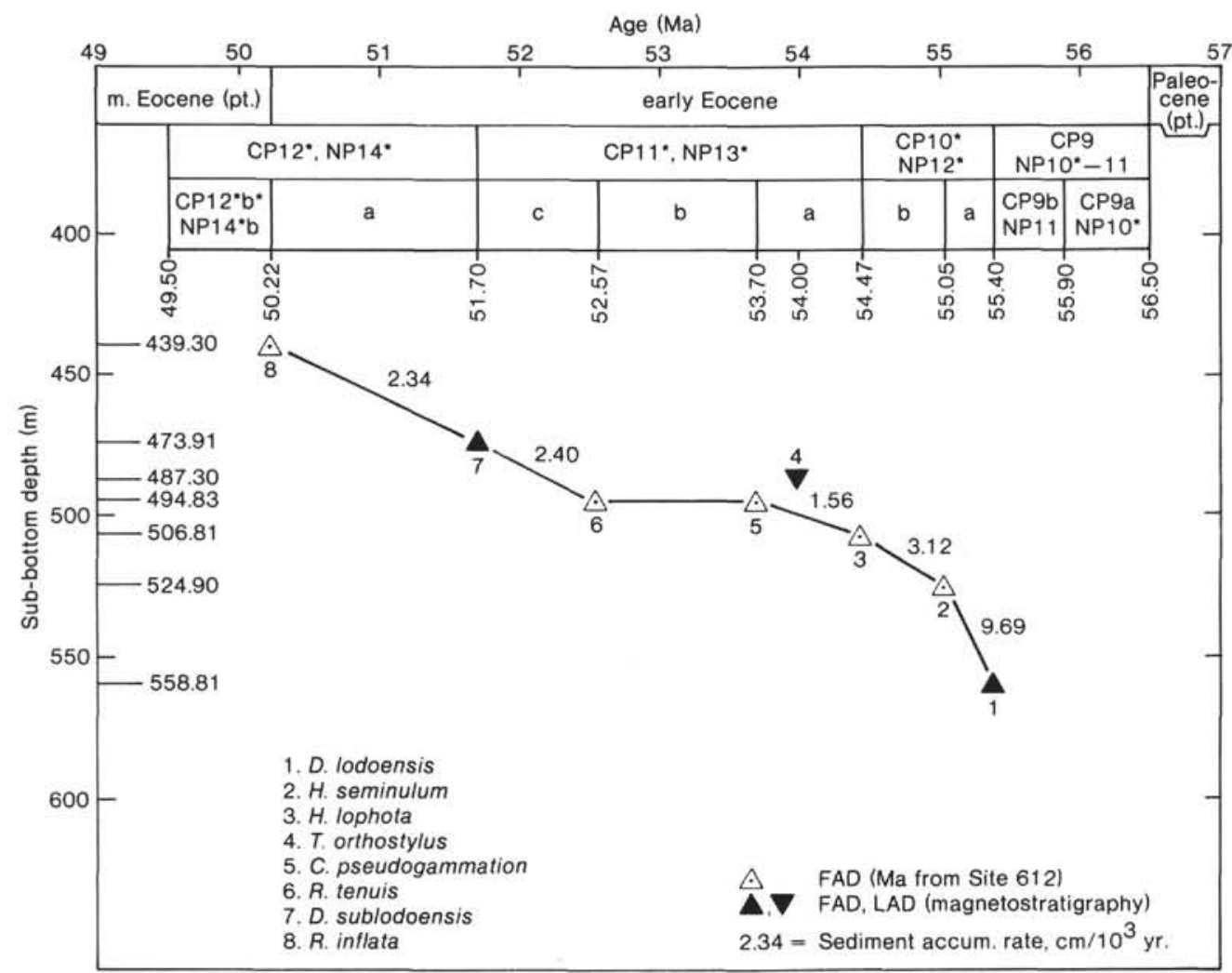

Figure 7. Time-depth plot of nannofossil datums at Site 613, New Jersey rise. Asterisk ${ }^{*}$ ) superscripts on CP and NP codes indicate revision of original zone or subzone. FAD, first-appearance datum; LAD, last-appearance datum. Curve is based on ages of datums determined from magnetostratigraphy (closed triangles; Shackleton, 1984) and those determined from curve for Site 612 (open triangles). LAD of T. orthostylus not used to plot curve, because it appears to be reworked above its usual stratigraphic level in the Site 613 section. Sediment accumulation rates for oldest part of section (CP10*a; NP12*a) is twice that of the same interval at Site 612, New Jersey slope, and suggests contemporaneous downslope transport at Site 613. An unconformity is present, possibly representing an episode of mass wasting, and the hiatus encompasses at least Subzone CP11*b (NP13*b) and possibly parts of Subzones CP11*a and CP11*c (NP13*a and NP13*c); see Figure 5. Sediment accumulation rates between nannofossil datums 3 and 5 and between 6 and 7 are minimums because duration of hiatus is unknown. Sediment accumulation rate for upper part of section $\left(\mathrm{CP} 12^{*} \mathrm{a} ; \mathrm{NP}_{14 * a}\right)$ is comparable to that of same interval at Site 612.

missing there. A time-depth curve based solely on data from Site 613 (i.e., drawn between nannofossil datums dated by magnetostratigraphy) (Fig. 7) would have produced somewhat different accumulation rates for the lower part of the section and older ages for the projected first occurrences of $C$. pseudogammation and $R$. tenuis. The low slope of a line drawn between the last occurrence of T. orthostylus and the first occurrence of D. sublodoensis (a span of $2.3 \mathrm{~m}$.y.) may have suggested the presence of an unconformity in the section, but there would be no proof of it.

\section{Duration of Lower Eocene Calcareous Nannofossil Biozones}

The ages of several nannofossil datums taken from the literature, and of those determined in this study, establish a preliminary chronology for nannofossil biozones of the lower Eocene. Ages for the Paleocene/Eocene and lower Eocene/middle Eocene boundaries generally are chosen from data presented in previous studies. At present, there is no definitive Paleocene/Eocene boundary based on calcareous nannofossils or planktonic foramini- fers, but the NP9/NP10 and the P6a/P6b zonal boundaries generally are used as close approximations. The Paleocene/Eocene boundary was recognized by some workers as the boundary between the Apectodinium hyperacanthum and Wetzeliella astra dinocyst Zones in the London Clay Formation of the London and Hampshire basins (see Berggren, Kent, and Flynn, 1985, fig. 4, and references therein, for a discussion of this boundary). It is not possible to correlate this datum with nannofossil biozones in northwestern Europe, owing to the lack of fossils in shallow-water deposits and to unconformities in the section (Aubry, 1983, 1985). So far, there is no positive correlation anywhere between the Paleocene/Eocene dinocyst zonal boundary and the NP9/NP10 boundary; but strata that can be assigned both to NP10 and to the $W$. astra Zone were recovered at DSDP Hole 117A on the Rockall Plateau (Morton et al., 1983).

The age of the Paleocene/Eocene boundary, defined by the dinocyst zonal boundary, was interpreted to be about $56.5 \mathrm{Ma}$ on the basis of $\mathrm{K}$-Ar radiometric dating of East Greenland basalts that lie between strata containing dinoflagellate assemblages characteristic of the 
Paleocene/Eocene boundary interval in northwestern Europe (Berggren, Kent, and Flynn, 1985). By contrast, the Paleocene/Eocene boundary, as defined by the planktonic foraminifer P6a/P6b zonal boundary, was drawn at the last occurrence of Morozovella velascoensis and dated as $57.8 \mathrm{Ma}$ on the basis of paleomagnetic chronology; the nannofossil NP9/NP10 boundary was placed at the first occurrence of Tribrachiatus nunnii and biostratigraphically correlated with the P6a/P6b boundary and the CP8/CP9 boundary (Berggren, Kent, and Flynn, 1985; Berggren, Kent, Flynn, and Van Couvering, 1985). We saw in an earlier section, however, that the NP9/NP10 boundary and the CP8/CP9 boundary are defined by different nannofossil datums and therefore probably are not coeval (Fig. 2). The first occurrence of D. diastypus, which both defines the base of Zone CP9 (NP10*-11) and is an approximation of the Paleocene/ Eocene boundary, was interpreted to be at about 56.5 Ma (Shackleton, 1984; Berggren, Kent, and Flynn, 1985), the same age as the radiometrically dated East Greenland basalts mentioned above. This evidence leaves us the choice of accepting for the Paleocene/Eocene boundary (1) an age of $56.5 \mathrm{Ma}$ based on dinocyst stratigraphy, the age of East Greenland basalts, and the age of the first occurrence of $D$. diastypus, or (2) an age of $57.8 \mathrm{Ma}$ estimated for the P6a/P $6 \mathrm{~b}$ boundary on the basis of paleomagnetic chronology.

The lower Eocene/middle Eocene boundary is also poorly defined in the stratotype areas of northwestern Europe. This boundary was discussed by Berggren, Kent, and Flynn (1985) and drawn at the base of Zone P10, defined by the first occurrence of the foraminifer Hantkenina aragonensis and correlated by them with the base of Subzone CP12b, defined by the first occurrence of Rhabdosphaera inflata. Although it is unlikely that these boundaries actually coincide, the first-occurrence datum of $R$. inflata (base of the Rhabdosphaera inflata Subzone; CP12*b*; NP14*b) is chosen in the present study to represent the top of the lower Eocene with an age of $50.2 \mathrm{Ma}$ (Fig. 6). The choice here of an age of $51.7 \mathrm{Ma}$, as given by Shackleton (1984), for the first occurrence of D. sublodoensis (base of the D. kuepperi Subzone; CP12*a; NP14*a) dictates a younger age $(50.2 \mathrm{Ma})$ for the base of CP12*b* (arbitrary lower Eocene/middle Eocene boundary) than that of about $52 \mathrm{Ma}$ chosen by Berggren, Kent, and Flynn (1985, fig. 5) and Berggren, Kent, Flynn, and Van Couvering (1985, table 3).

The arguments set forth above are the basis for interpreting the duration of nannofossil zones in the lower Eocene sequence at Site 612 (Fig. 6). The base of the lower Eocene is drawn at the base of CP9 (NP10*-11), the $D$. diastypus Zone, with an age of approximately $56.5 \mathrm{Ma}$; and the top of the lower Eocene is placed at the base of CP12*b* (NP14*b), the R. inflata Subzone, with an age of approximately $50.2 \mathrm{Ma}$, suggesting a duration of 6.3 m.y. for the lower Eocene as used here. Within the lower Eocene, the boundary between Subzones CP9a and CP9b (NP10* and NP11) is the last occurrence of Tribrachiatus contortus. The age of this datum was interpreted to be approximately $55.9 \mathrm{Ma}$ at DSDP Site 528, and its age is tentative; but the data from Site 528 appear to be better constrained than those from Site 527, where recovery was poor and paleomagnetic information lacking at this level (Shackleton, 1984; Berggren, Kent and Flynn, 1985). The age of the base of CP10* (NP12*) at the first occurrence of $D$. lodoensis is approximately 55.4 Ma. Thus, Subzones CP9a (NP10*) and CP9b (NP11) span 0.6 and 0.5 m.y., respectively.

From the previous discussion of nannofossil datums (Fig. 6), we see that the duration of $\mathrm{CP} 10^{*}\left(\mathrm{NP} 12^{*}\right)$ was approximately 0.9 m.y., with Subzones CP10*a and CP10*b (NP12*a and NP12*b) lasting 0.4 and 0.5 m.y., respectively. Similarly, the duration of CP11* (NP13*) was about 2.8 m.y., with Subzones CP11*a, CP11*b, and $\mathrm{CP} 11^{*} \mathrm{c}\left(\mathrm{NP} 13^{*} \mathrm{a}, \mathrm{NP} 13^{*} \mathrm{~b}\right.$, and $\left.\mathrm{NP}^{2} 3^{*} \mathrm{c}\right)$ lasting 0.8 , 1.1 , and 0.9 m.y., respectively. The highest subzone of the lower Eocene, CP12*a (NP14*a), had a duration of 1.5 m.y., based on the age of the first occurrence of $D$. sublodoensis ( $51.7 \mathrm{Ma}$ ) from Site 527 (Shackleton, 1984) and the age of the first occurrence of $R$. inflata (50.2 $\mathrm{Ma}$ ) as interpreted from projection onto the time-depth curve for Site 612.

\section{DISCUSSION AND CONCLUSIONS}

The lower Eocene section at DSDP Site 612 beneath the New Jersey slope is calcareous nannofossil limestone approximately $198 \mathrm{~m}$ thick. The orderly succession of species ranges through time suggests that there are no major unconformities in this section. The thickness of the lower Eocene strata and the good preservation of nannofossils have made it possible to determine accurately the ranges of recognized biostratigraphic marker species and of other distinctive species that have been employed here to delineate new subzones and to refine the existing Martini (NP zones) and Bukry-Okada (CP zones) zonation schemes. A similar lower Eocene section at nearby DSDP Site 613, beneath the New Jersey rise, is approximately $139 \mathrm{~m}$ thick, and contains an unconformity representing the absence of newly defined Subzone CP11*b (NP13*b) and a hiatus of at least of 1.1 m.y. duration.

Comparison of the New Jersey slope and rise sections with other lower Eocene sections reveals that oceanic sequences generally are only tens of meters thick. Thus, there is a good chance that an appreciable part of the deep-sea record will be removed during episodes of erosion. Even if biozones are not completely removed by such processes, the ranges of individual species with respect to one another may be misrepresented in a relatively thin record containing unconformities.

Epicontinental deposits of early Eocene age are often thicker than those found in ocean basins, but are even more susceptible to removal by erosion. The sequence of nannofossil biozones in northwestern Europe is incomplete, and the absence of Zone NP10 and parts of other biozones is attributable to unconformities, to diagenesis, and to the lack of appropriate fossils in shallow-water deposits (Aubry, 1983, 1985). Indeed, the incompleteness of the biostratigraphic record in Europe has complicated the correlation of biozones with stage boundaries. By contrast, strata equivalent to the lower Eocene Zones CP10* and CP11* (NP12* and NP13*) consist of ap- 
proximately $320 \mathrm{~m}$ of deeper-water deposits of the Lodo Formation at its type locality in California. This thick section was the object of the classic nannofossil study of Bramlette and Sullivan (1961) in which the ranges of numerous species were delineated.

Two widely accepted zonation schemes form the Cenozoic nannofossil biostratigraphic framework. Lower Eocene nannofossil marker species employed in the standard (high-latitude) zonation (Martini, 1971) and in the tropical (low-latitude) zonation (Bukry, 1973, 1975; Okada and Bukry, 1980) are in many cases identical and the corresponding biozones coeval. Moreover, the reference sections for Martini's Zones NP12 and NP13 (CP10 and $\mathrm{CP} 11)$ are in Cuba, a low-latitude locality, whereas other reference sections are in Switzerland and California. The Bukry-Okada zones were based on oceanic strata. Both the Martini and the Bukry-Okada zonations can be applied with equal effectiveness to the lower Eocene strata cored at Sites 612 and 613 on the New Jersey slope and rise, and this is generally also true for the lower Eocene deposits of northwestern Europe, the Gulf Coast, and California.

The emendation of CP and NP zones in the present study retains established zonal and subzonal names but slightly alters alphanumeric codes by appending an asterisk $\left({ }^{*}\right)$ to indicate revision. Several zonal boundaries are revised and new subzones established. The Martini and Bukry-Okada biozonal boundaries are revised in this study as follows:

1. The base of Zone NP10 (Tribrachiatus contortus Zone) is recognized here as the first occurrence of Discoaster diastypus rather than the first occurrence of Tribrachiatus nunnii. This follows the revision of Bukry (1975) and results in the equivalence of Subzones NP10* and CP9a, the Tribrachiatus contortus Subzone.

2. The boundaries between CP10 and CP11 and between NP12 and NP13 are revised to be coeval. The CP10*/CP11* boundary datum, formerly the first occurrence of Coccolithus crassus, and the NP12*/NP13* boundary datum, formerly the last occurrence of Tribrachiatus orthostylus, are now the first occurrence of Helicosphaera lophota.

3. The top of Subzone CP12*b* (NP14*b), formerly the first occurrence of Nannotetrina fulgens, is now the last occurrence of Rhabdosphaera inflata. The first occurrence of $N$. fulgens is a secondary datum to mark this boundary.

Five new subzones are erected within the newly emended CP10* and CP11* (NP12* and NP13*) zones.

1. The datums that delineate these subzones are the first occurrences, from oldest to youngest, of Discoaster lodoensis, Helicosphaera seminulum, Helicosphaera lophota, Cyclicargolithus pseudogammation, Rhabdosphaera tenuis, and Discoaster sublodoensis.

2. The five new subzones are as follows: CP10*a (NP12*a)-Lophodolithus nascens Subzone; CP10*b (NP12*b)-Helicosphaera seminulum Subzone; CP11*a (NP13*a)-Helicosphaera lophota Subzone; CP11*b (NP13*b) - Cyclicargolithus pseudogammation Subzone; CP11*c (NP13*c)-Rhabdosphaera tenuis Subzone.
At Site 612, a time-depth curve is based on several dated biostratigraphic events from previous studies (Shackleton, 1984; Berggren, Kent, and Flynn, 1985): first occurrences of Discoaster lodoensis (55.4 Ma) and Discoaster sublodoensis (51.7 Ma); last occurrences of Tribrachiatus orthostylus $(54.0 \mathrm{Ma})$ and Rhabdosphaera inflata (approx. $49.5 \mathrm{Ma}$, the age of the first occurrence of Nannotetrina fulgens). The time-depth plot reveals that the sediment accumulation rate during deposition of CP10* (NP12*) was $4.9 \mathrm{~cm} / 10^{3} \mathrm{yr}$. This rate declined to $3.7 \mathrm{~cm} / 10^{3} \mathrm{yr}$. during deposition of CP11* (NP13*), and thence to $2.81 \mathrm{~cm} / 10^{3}$ yr. during CP12* (NP14*).

The ages of previously undated nannofossil datums are approximated by projecting their stratigraphic depths onto the time-depth curve at Site 612. By this method, the preliminary ages of first-occurrence datums are as follows: Helicosphaera seminulum, 55.0 Ma; Helicosphaera lophota, $54.5 \mathrm{Ma}$; Cyclicargolithus pseudogammation, $53.7 \mathrm{Ma}$; Rhabdosphaera tenuis, $52.6 \mathrm{Ma}$; and Rhabdosphaera inflata, $50.2 \mathrm{Ma}$.

At Site 613, a time-depth curve, based in part on ages of nannofossil datums determined at Site 612, reveals the presence of at least a 1.1-m.y. hiatus representing Subzone CP11*b (NP13*b) and a sediment accumulation rate $\left(9.7 \mathrm{~cm} / 10^{3} \mathrm{yr}\right.$.) during subzone CP10*a (NP12*a) time that is twice that at Site 612. These departures from the smooth time-depth trend displayed at Site 612 probably represent the effects of relatively short-lived masswasting phenomena on the early Eocene continental slope and rise. Also at Site 613, but on a much smaller scale and higher in the section within the middle Eocene Rhabdosphaera inflata Subzone (CP12*b*; NP14*b), there is a $78-\mathrm{cm}$ interval of lower Eocene (CP12*a; NP14*a) slumped sediment. The slump lies between depths of 437.92 and $438.70 \mathrm{~m}$, and was identified both by the abrupt disappearance and later reappearance of Rhabdosphaera inflata and by a color change in the strata and distorted bedding.

\section{REFERENCES}

Aubry, M. P., 1983. Biostratigraphie du Paléogène épicontinental de l'Europe du nord-ouest, étude fondée sur les nannofossiles calcaires. Docum. Lab. Geol. Lyon, 89.

, 1985. Northwestern European magnetostratigraphy, biostratigraphy, and paleogeography: Calcareous nannofossil evidence. $\mathrm{Ge}$ ology, 13:198-202.

Berggren, W. A., and Aubert, J., 1983. Paleogene benthic foraminiferal biostratigraphy and paleobathymetry of the central ranges of California. U.S. Geol. Surv. Prof. Pap., 1213:4-21.

Berggren, W. A., Kent, D. V., and Flynn, J. J., 1985. Paleogene geochronology and chronostratigraphy. In Snelling, N. (Ed.), Geochronology and the Geological Record. Geol. Soc. London Spec. Publ.

Berggren, W. A., Kent, D. V., Flynn, J. J., and Van Couvering, J. A., 1985. Cenozoic geochronology. Geol. Soc. Am. Bull., 96:14071418.

Bramlette, M. N., and Sullivan, F. R., 1961. Coccolithophorids and related nannoplankton of the early Tertiary in California. Micropaleontology, 7:129-188.

Brönnimann, P., and Rigassi, D., 1963. Contribution to the geology and paleontology of the area of the city of La Habana, Cuba, and its surroundings. Eclogae Geol. Helvet., 56:193-480.

Brönnimann, P., and Stradner, H., 1960. Die Foraminiferen- und Discoasteridenzonen von Kuba and ihre interkontinentale Korrelation. Erdoel Z., 76:364-369. 
Bukry, D., 1971. Cenozoic calcareous nannofossils from the Pacific Ocean. San Diego Soc. Nat. Hist. Trans., 16:303-328.

1973. Low-latitude coccolith biostratigraphic zonation. In Edgar, N. T., Saunders, J. B., et al., Init. Repts. DSDP, 15: Washington (U.S. Govt. Printing Office), 685-703.

1975. Coccolith and silicoflagellate stratigraphy, northwestern Pacific Ocean, Deep Sea Drilling Project Leg 32. In Larson, R. L., Moberly, R., et al., Init. Repts. DSDP, 32: Washington (U.S. Govt. Printing Office), 677-692.

1978. Biostratigraphy of Cenozoic marine sediment by calcareous nannofossils. Micropaleontology, 24:44-60. 1981. Pacific Coast coccolith stratigraphy between Point Conception and Cabo Corrientes, Deep Sea Drilling Project Leg 63. In Yeats, R. S., Haq. B. U., et al., Init. Repts. DSDP, Washington (U.S. Govt. Printing Office), 445-471.

1985. Numerical ages of Cenozoic biostratigraphic datum levels. Results of South Atlantic Leg 73 drilling: Discussion and reply. Geol. Soc. Am. Bull., 96:813-814.

Bukry, D., Brabb, E. E., and Vedder, J. G., 1977. Correlation of Tertiary nannoplankton assemblages from the Coast and Peninsular ranges of California. Venezuela Ministerio Minas Hidrocarburos, Geol. Bol., Spec. Publ., 7:1461-1483.

Bukry, D., and Percival, S. F., 1971. New Tertiary calcareous nannofossils. Tulane Stud. Geol. Paleontol., 8:123-146.

Bybell, L. M., and Gibson, T. G., 1985. The Eocene Tallahatta Formation of Alabama and Georgia: Its lithostratigraphy, biostratigraphy, and bearing on the age of the Claibornian Stage. U.S. Geol. Surv. Bull., 1615.

Gartner, S., 1970. Phylogenetic lineages in the lower Tertiary coccolith genus Chiasmolithus. Proc. North Am. Paleontol. Conv. 1969, Pt. G, pp. $930-957$.

, 1971. Calcareous nannofossils from the Joides Blake Plateau cores, and revision of Paleogene nannofossil zonation. Tulane Stud. Geol. Paleontol., 8:101-121.

Hay, W. W., 1964. Utilisation stratigraphique des discoasterides pour la zonation du Paléocène et l'Eocène inférieur. Mem. Bur. Rech. Geol. Minieres, 28:885-889.

1967. Zonation of the middle-upper Eocene interval. In Hay, W. W., Mohler, H. P., Roth, P. R., Schmidt, R. R., and Boudreaux, J. E., Calcareous Nannoplankton Zonation of the Cenozoic of the Gulf Coast and Caribbean-Antillean Area, and Transoceanic Correlation. Gulf Coast Assoc. Geol. Soc. Trans., 17:438439.

Hay, W. W., and Mohler, H. P., 1967. Calcareous nannoplankton from early Tertiary rocks at Point Labau, France, and Paleocene-early Eocene correlations. J. Palontol., 41:1505-1541.

Hazel, J. E., Edwards, L. E., and Bybell, L. M., 1984. Significant unconformities and the hiatuses represented by them in the Paleogene of the Atlantic and Gulf Coastal Province. In Schlee, J. (Ed.), Interregional Unconformities. Mem. Am. Assoc. Pet. Geol., 36:5966.

Hsü, K. J., 1985. Numerical ages of Cenozoic biostratigraphic datum levels. Results of South Atlantic Leg 73 drilling: Discussion and reply. Geol. Soc. Am. Bull., 96:814-815.

Hsü, K. J., LaBrecque, J. L., Percival, S. F., Wright, R., Gombos, A. M., et al., 1984. Numerical ages of Cenozoic biostratigraphic datum levels: Results of South Atlantic Leg 73 drilling. Geol. Soc. Am. Bull., 95:863-876.

International Subcommission on Stratigraphic Classification (ISSC), 1976. International Stratigraphic Guide (H. D. Hedberg, Ed.): New York (John Wiley and Sons).
Martini, E., 1970. Standard Paleogene calcareous nannoplankton zonation. Nature, 266:560-561.

1971. Standard Tertiary and Quaternary calcareous nannoplankton zonation. In Farinacci, A. (Ed.), Proc. Second Int. Conf. Planktonic Microfossils: Rome (Edizioni Tecnoscienza), pp. 739785.

Mohler, H. P., and Hay, W. W., 1967. Zonation of the Paleocenelower Eocene interval. In Hay, W. W., Mohler, H. P., Roth, P. R., Schmidt, R. R., and Boudreaux, J. E., Calcareous Nannoplankton Zonation of the Cenozoic of the Gulf Coast and Caribbean-Antillean Area, and Transoceanic Correlation. Gulf Coast Assoc. Geol. Soc. Trans., 17:432-438.

Monechi, S., and Thierstein, H. R., in press. Late Cretaceous-Paleogene nannofossil and magnetostratigraphic correlation in the Umbrian Appenines. Geol. Soc. Am. Bull.

Morton, A. A., Backman, J., and Harland, R., 1983. A reassessment of the stratigraphy of DSDP Hole 117A, Rockall Plateau: Implications for the Palocene-Eocene boundary in N.W. Europe. Newsl. Stratigr., 12:104-111.

Müller, C., 1974. Calcareous nannoplankton, Leg 25 (western Indian Ocean). In Simpson, E. S. W., Schlich, R., et al., Init. Repts. DSDP, 25: Washington (U.S. Govt. Printing Office) 579-633.

North American Commission on Stratigraphic Nomenclature, 1983. North American Stratigraphic Code. Am. Assoc. Petrol. Geol. Bull., 67:841-875.

Okada, H., and Bukry, D., 1980. Supplementary modification and introduction of code numbers to the low-latitude coccolith biostratigraphic zonation (Bukry, 1973; 1975). Mar. Micropaleontol., 5: 321-325.

Okada, H., and Thierstein, H. R., 1979. Calcareous nannoplanktonLeg 43, Deep Sea Drilling Project. In Tucholke, B. E., Vogt, P. R., et al., Init. Repts. DSDP, 43: Washington (U.S. Govt. Printing Office), 507-573.

Perch-Nielsen, K., 1977. Albian to Pleistocene calcareous nannofossils from the western South Atlantic, DSDP Leg 39. In Supko, P. R., Perch-Nielsen, K., et al., Init. Repts. DSDP, 39: Washington (U.S. Govt. Printing Office), 699-823.

Percival, S. F., 1984. Late Cretaceous to Pleistocene calcareous nannofossils from the South Atlantic, Deep Sea Drilling Project Leg 73. In Hsü, K. J., LaBrecque, J. L., et al., Init. Repts. DSDP, 73: Washington (U.S. Govt. Printing Office), 391-424.

Poore, R. Z., 1976. Microfossil correlation of California lower Tertiary sections: A comparison. U.S. Geol. Surv. Prof. Pap., 743-F.

Romein, A. J. T., 1979. Lineages in early Palogene calcareous nannoplankton. Utrecht Micropalentol. Bull., 22.

Shackleton, N. J., 1984. Accumulation rates in Leg 74 sediments. In Moore, T. C., Jr., Rabinowitz, P. D., et al., Init. Repts. DSDP, 74: Washington (U.S. Govt. Printing Office), 621-644.

Sullivan, F. R., 1965. Lower Tertiary nannoplankton from the California Coast Ranges, II, Eocene. Univ. Calif. Publ. Geol. Sci., 53.

Warren, A. D., 1983. Lower Tertiary nannoplankton biostratigraphy in the central Coast Ranges, California. U.S. Geol. Surv. Prof. Pap., 1213:22-38.

Date of Initial Receipt: 14 August 1985 Date of Acceptance: 3 March 1986 


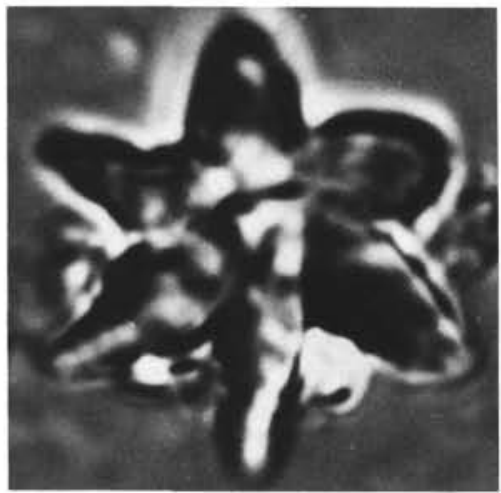

1

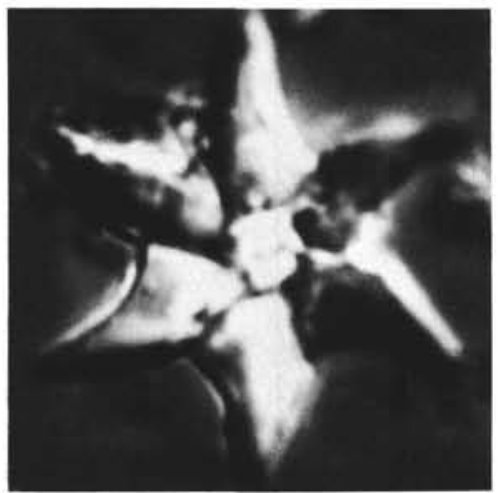

2

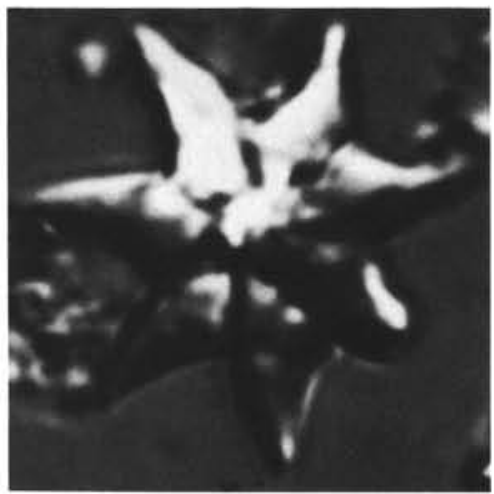

4

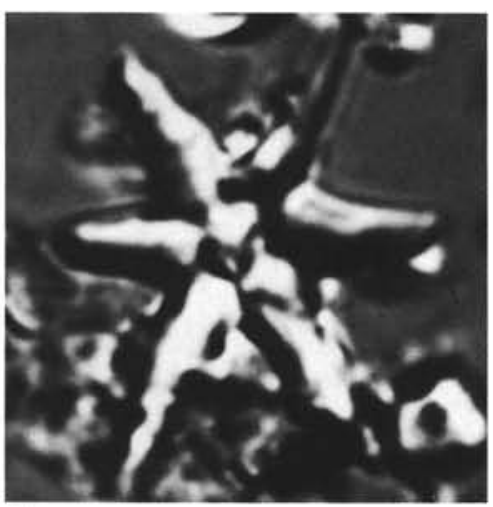

5

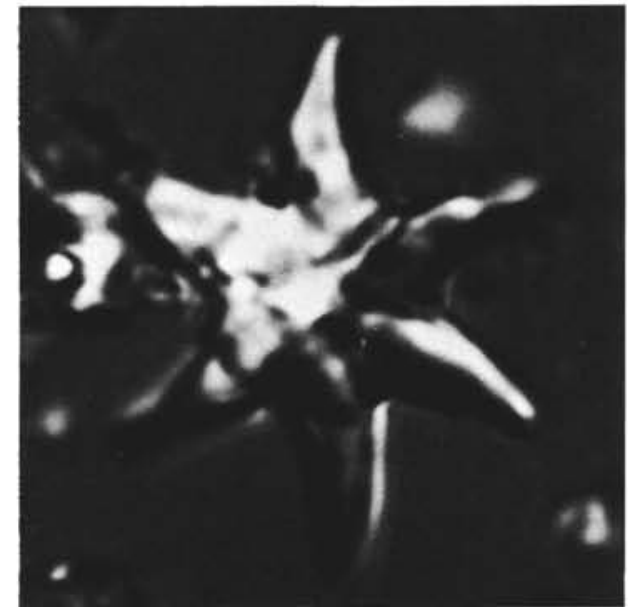

3

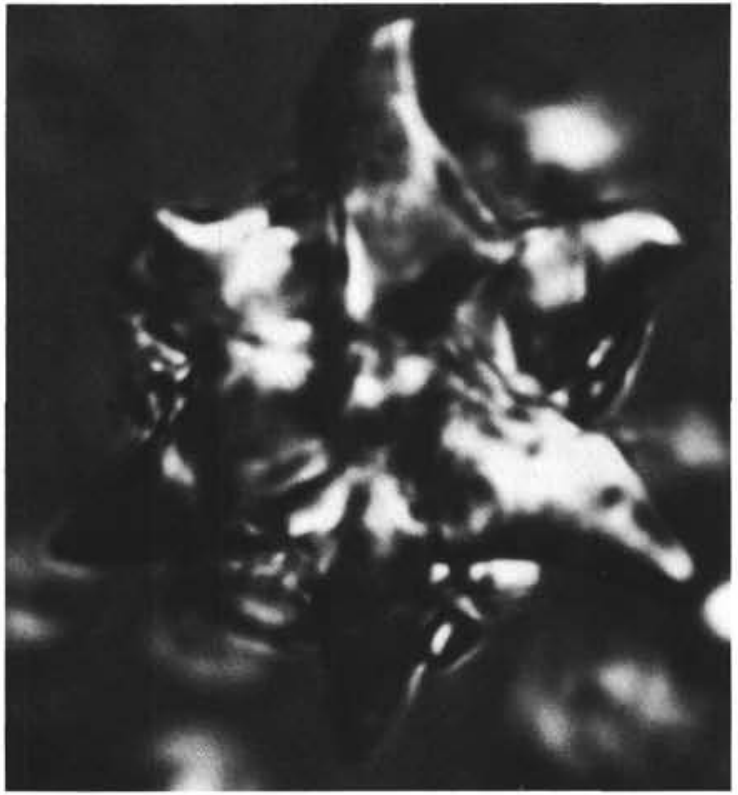

6

Plate 1. Light micrographs $(\times 3200)$ of lower Eocene nannofossils, New Jersey slope. 1, 2. Discoaster lodoensis Bramlette and Riedel, (1) Sample $612-59-1,20-22 \mathrm{~cm}(540.41 \mathrm{~m})$, L. nascens Subzone, R99N32, partly crossed nicols, (2) Sample 612-56,CC (521.00 m), H. seminulum Subzone, R103N6, interference contrast, blue filter. 3-5. Discoaster lodoensis Bramlette and Riedel, Sample 612-43-1, 20-22 cm (386.21 m), D. kuepperi Subzone, interference contrast, blue filter, (3) R110N15A, (4) R110N17A, (5) R110N13A. 6. Discoaster lodoensis Bramlette and Riedel, Sample $612-42-4,20-22 \mathrm{~cm}(381.11 \mathrm{~m})$, D. kuepperi Subzone, R110N4A, partly crossed nicols. 


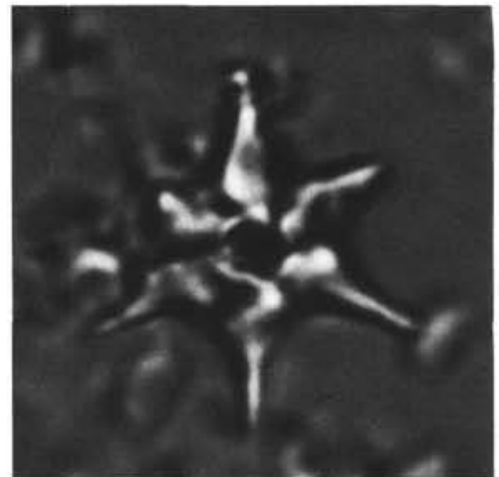

1

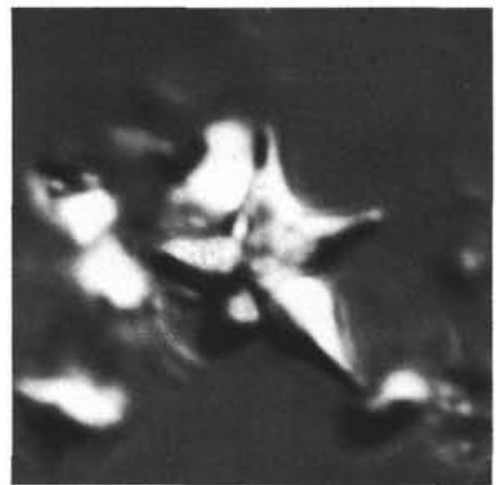

4

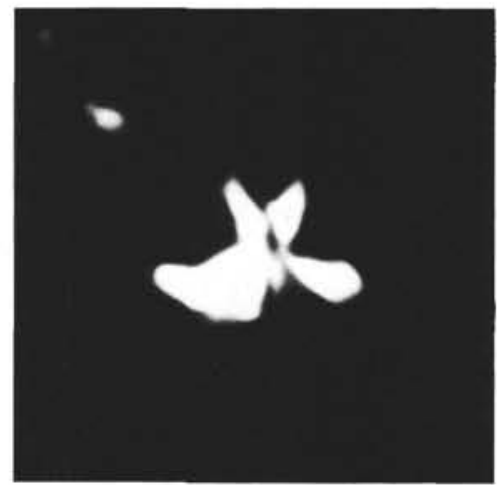

7

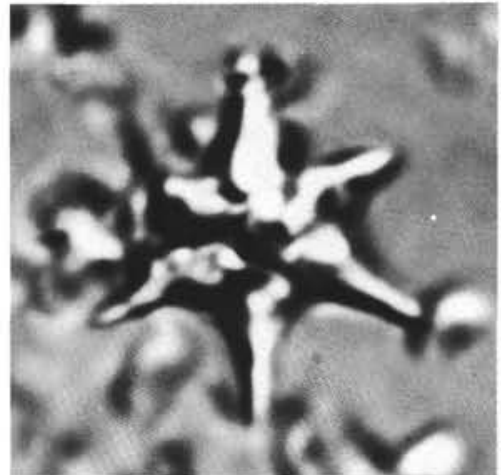

2

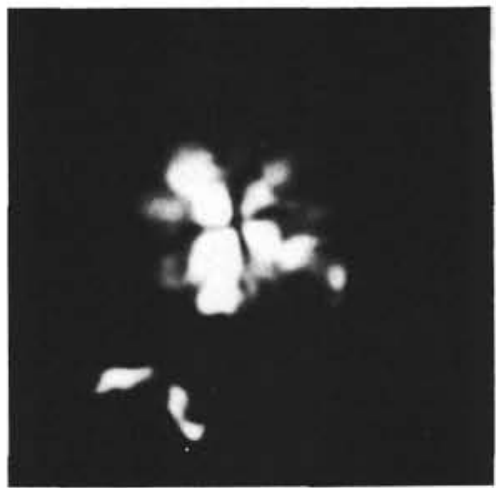

5

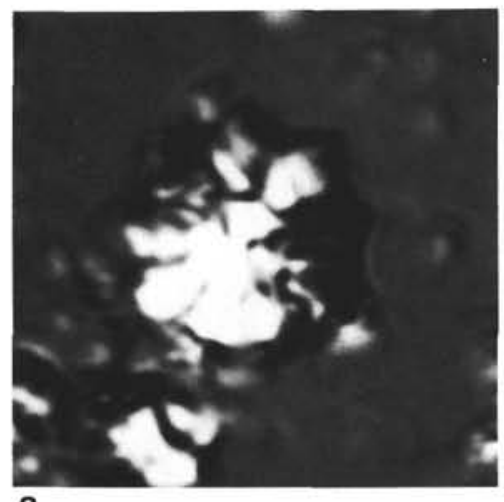

8

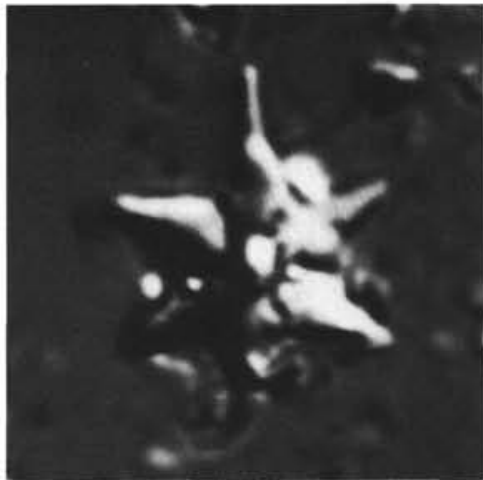

3

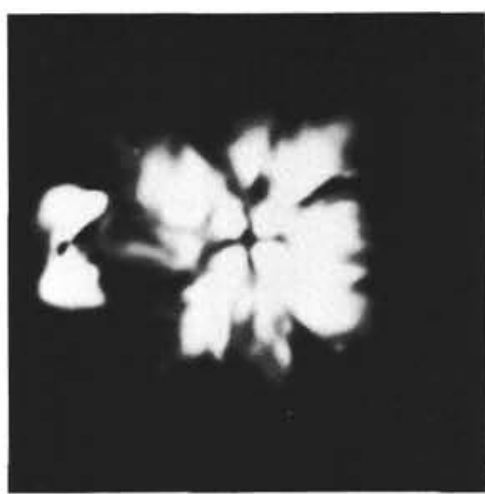

6

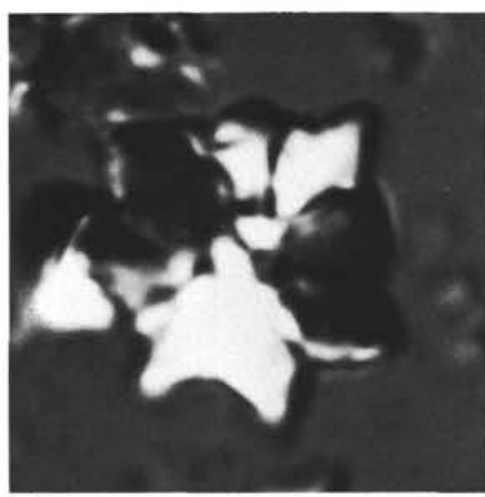

9

Plate 2. Light micrographs $(\times 3200)$ of lower Eocene (3-9) and middle Eocene $(1,2)$ nannofossils, New Jersey slope. 1, 2. Discoaster sublodoensis Bramlette and Sullivan, Sample 613-38,CC (339.34 m), R. inflata Subzone, (1) R109N16A, partly crossed nicols, blue filter, (2) R109N14A, interference contrast, blue filter, same specimen as (1). 3, 4. Discoaster sublodoensis Bramlette and Sullivan, both samples from D. kuepperi Subzone, (3) Sample 612-43-1, 20-22 cm (386.21 m), R111N16, interference contrast, blue filter, (4) Sample 612-42-4, 20-22 cm (381.11 m), R110N6A, partly crossed nicols, blue filter. 5, 8. Discoasteroides kuepperi (Stradner), Sample 612-55,CC (511.50 m), H. seminulum Subzone, (5) R92N22, crossed nicols, (8) R92N26, interference contrast, same specimen as (5). 7. Discoasteroides kuepperi (Stradner), Sample 612-51,CC (471.37 m), H. lophota Subzone, R107N35, crossed nicols, side view. 6, 9. Discoasteroides kuepperi (Stradner), Sample 612-43-1, 20-22 cm (386.21 m), D. kuepperi Subzone, (6) R110N19A, crossed nicols, (9) R110N20A, interference contrast, blue filter, same specimen as (6). 


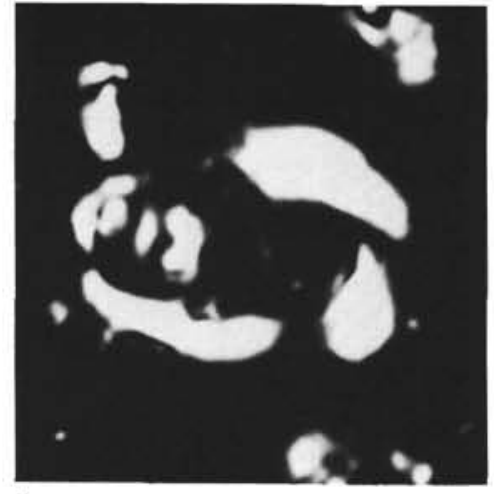

1

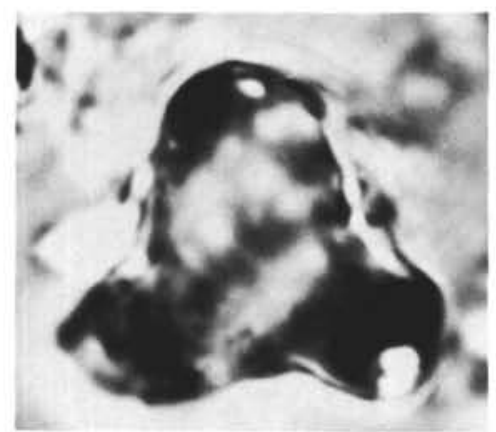

4

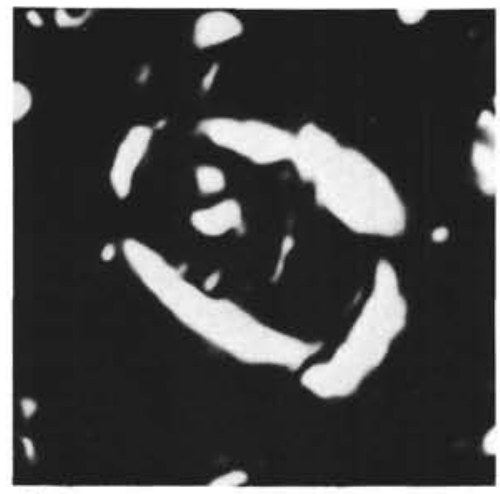

2

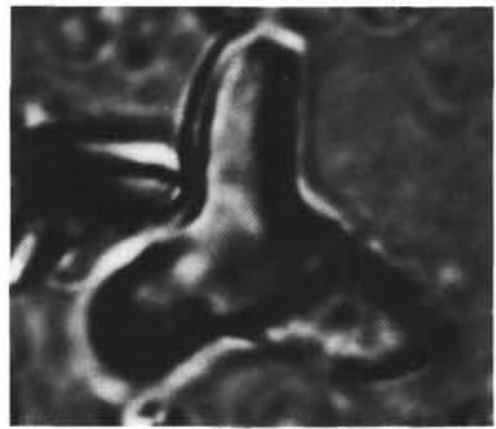

5

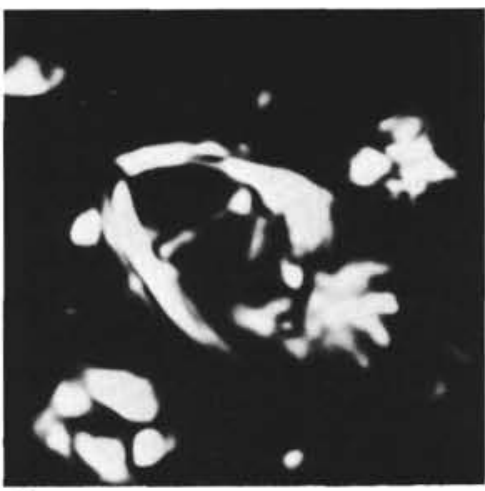

3

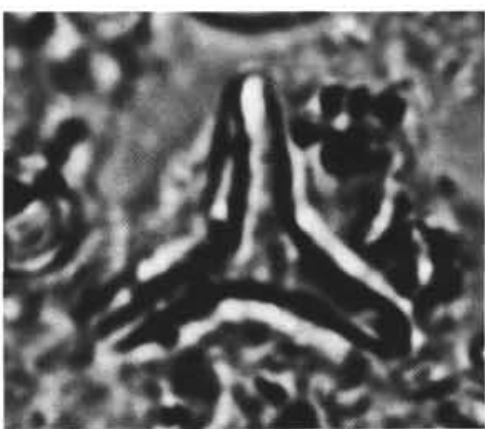

6

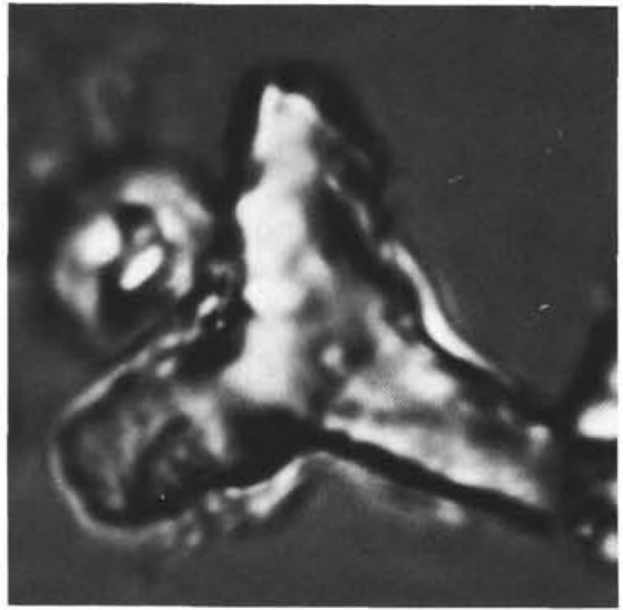

7

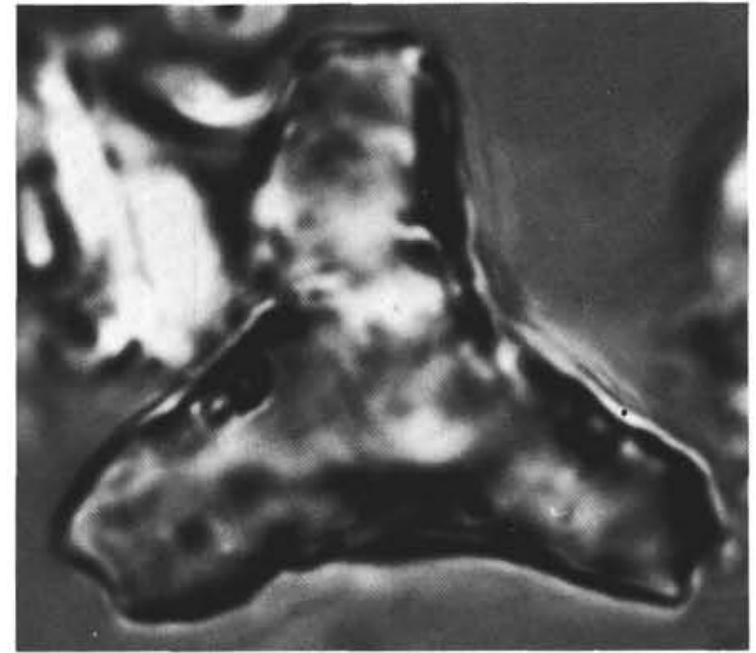

8

Plate 3. Light micrographs $(\times 3200)$ of lower Eocene nannofossils, New Jersey slope. 1, 2. Lophodolithus mochlophorus Deflandre, Sample 61247-2, 20-22 cm (426.31 m), R. tenuis Subzone, (1) R112N21, crossed nicols, (2) R112N23, crossed nicols, same specimen as (1). 3. Lophodolithus mochlophorus Deflandre, Sample 612-43,CC $(395.57 \mathrm{~m}), R$. tenuis Subzone, R109N21A, crossed nicols. 4, 7, 8. Tribrachiatus orthostylus Shamrai, from D. binodosus Subzone, (4) Sample 612-60-1, 50-52 cm (550.31 m), R98N17, interference contrast, blue filter, (7) Sample 61259,CC (549.80 m), R97N5, partly crossed nicols, (8) Sample 612-59,CC $(549.80 \mathrm{~m})$, R97N8, partly crossed nicols. 5, 6. Tribrachiatus orthostylus Shamrai, Sample $612-55$, CC $(511.50 \mathrm{~m}), H$. seminulum Subzone, (5) R90N22A, partly crossed nicols, blue filter, (6) R90N20A, partly crossed nichols, blue filter. 


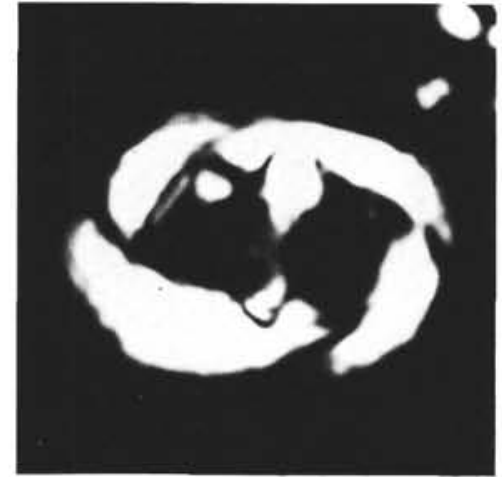

1

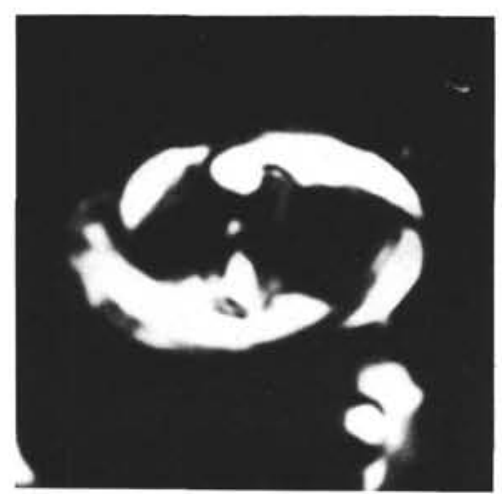

4

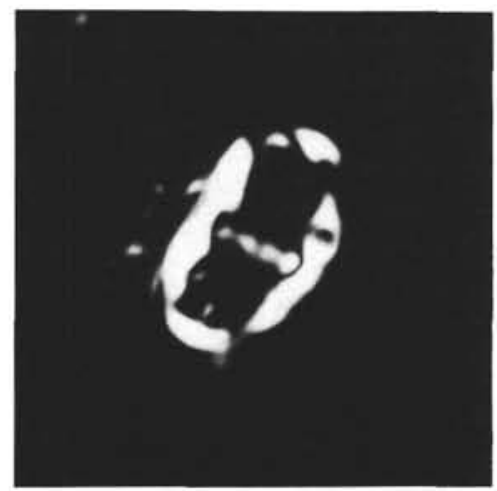

7

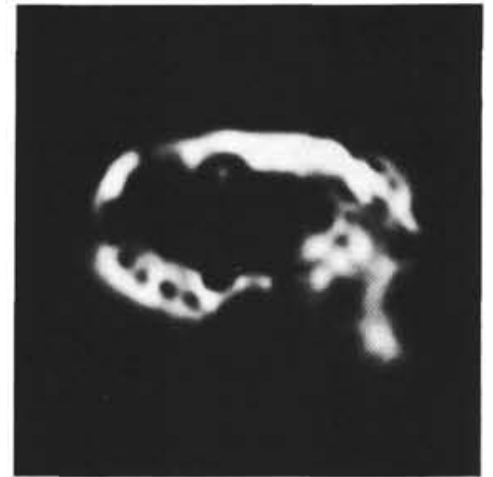

2

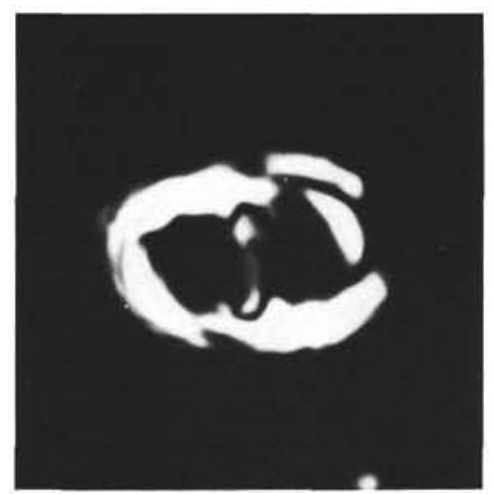

5

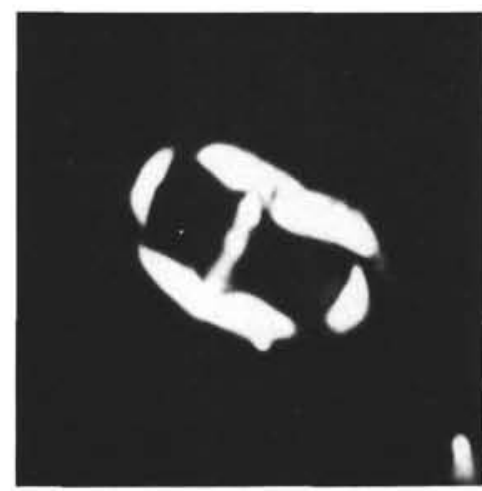

8

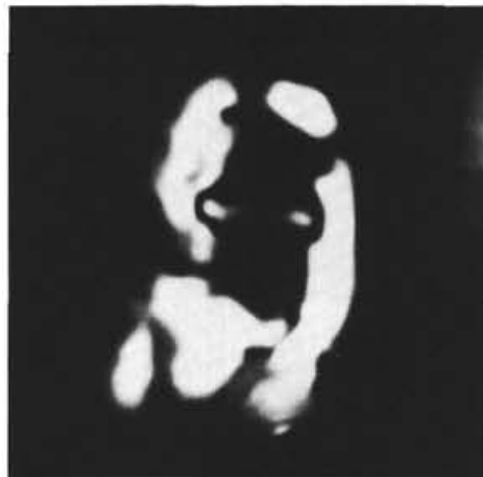

3

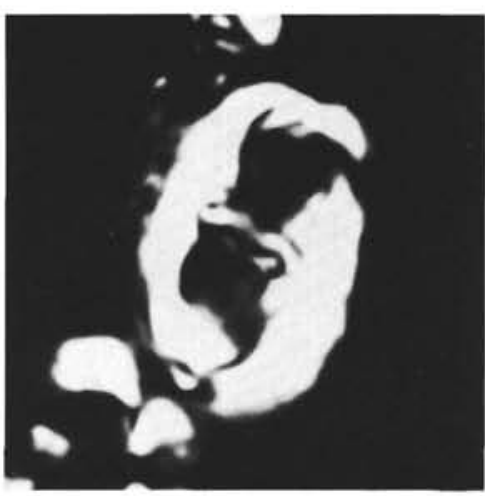

6

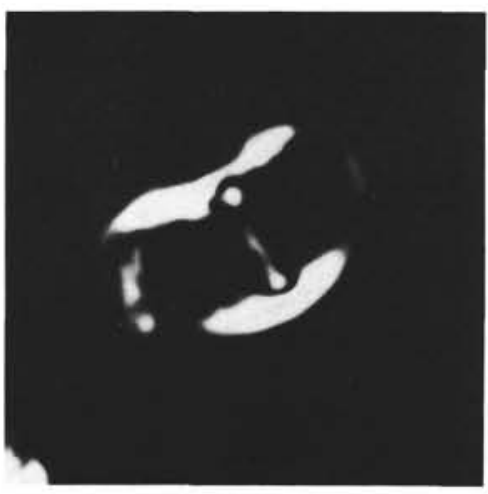

9

Plate 4. Light micrographs $(\times 3200)$ of lower Eocene nannofossils, New Jersey slope. (Crossed nicols.) 1. Lophodolithus nascens Bramlette and Sullivan, Sample 612-60-1, 50-52 cm (550.31 m), D. binodosus Subzone, R98N23. 2, 3. Lophodolithus nascens Bramlette and Sullivan, Sample 612-57-6, 20-22 cm (528.71 m), H. seminulum Subzone, (2) R101N20, (3) R101N17, same specimen as (2), rotated 90 ${ }^{\circ}$. 4, 5. Lophodolithus nascens Bramlette and Sullivan, from L. nascens Subzone, (4) Sample 612-59-6, 20-22 cm (547.91 m), R99N30A, (5) Sample 612-59-1, 20-22 cm (540.41 m), R100N24A. 6. Lophodolithus nascens Bramlette and Sullivan, Sample 612-55,CC (511.50 m), H. seminulum Subzone, R103N27. 79. Lophodolithus reniformis Bramlette and Sullivan, (7) Sample 612-57,CC (530.40 m), H. seminulum Subzone, R95N3, (8) Sample 612-55,CC (511.50 m), H. seminulum Subzone, R103N29, (9) Sample 612-54-4, 20-22 cm (497.21 m), H. lophota Subzone, R108N14. 


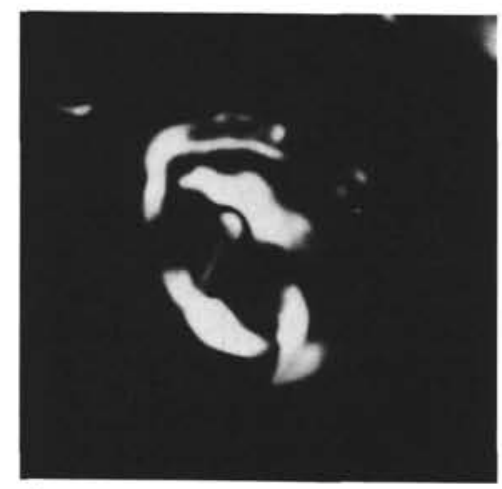

1

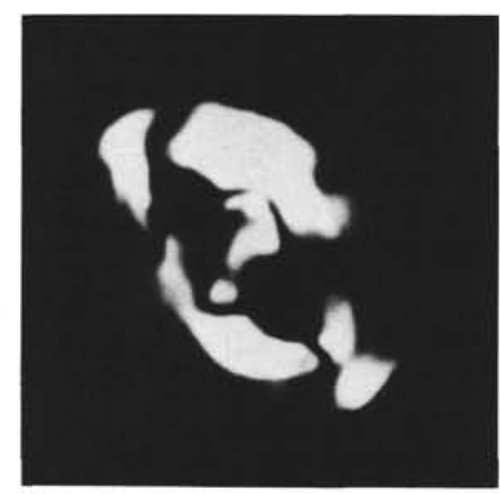

4

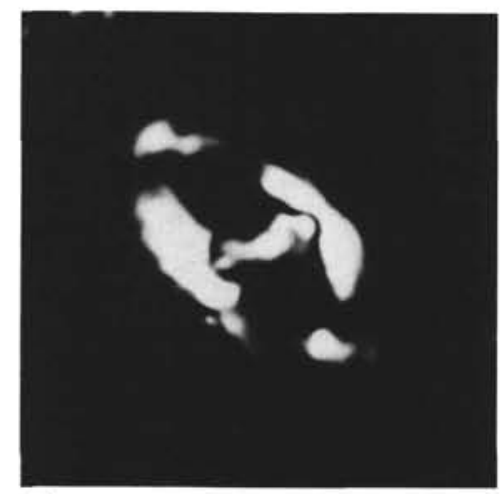

7

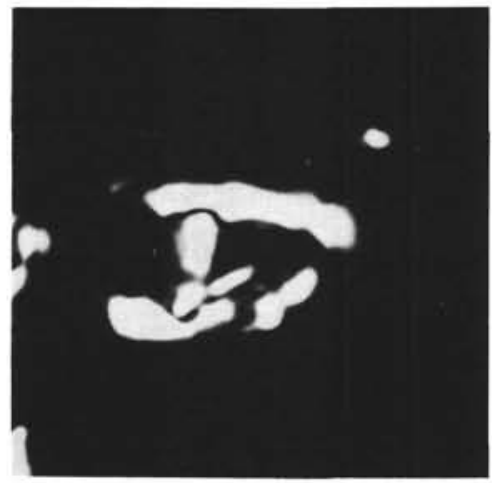

2

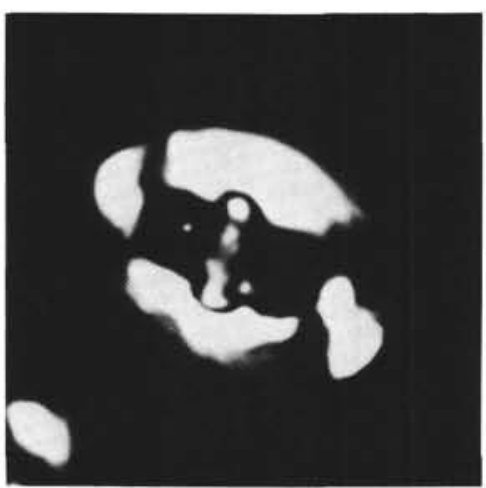

5

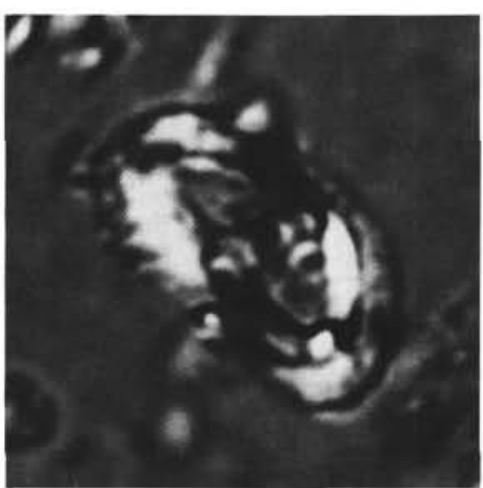

8

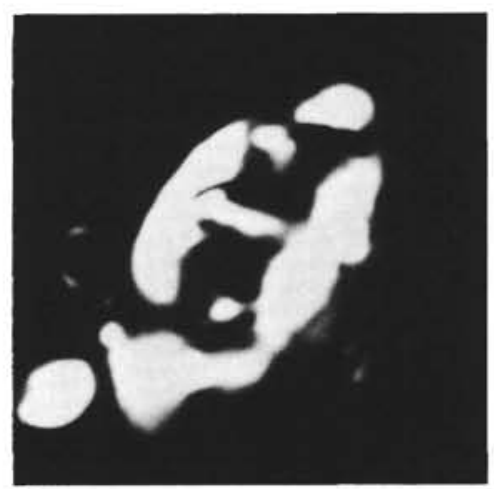

3

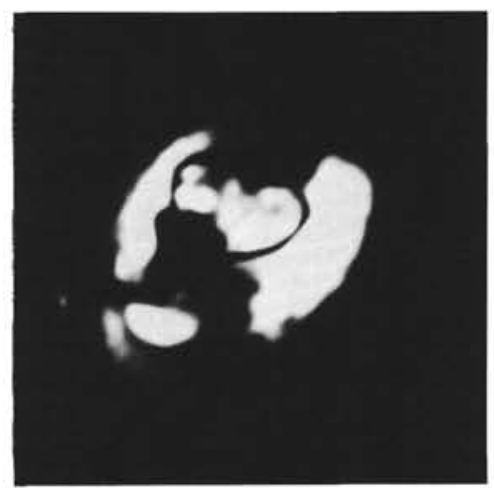

6

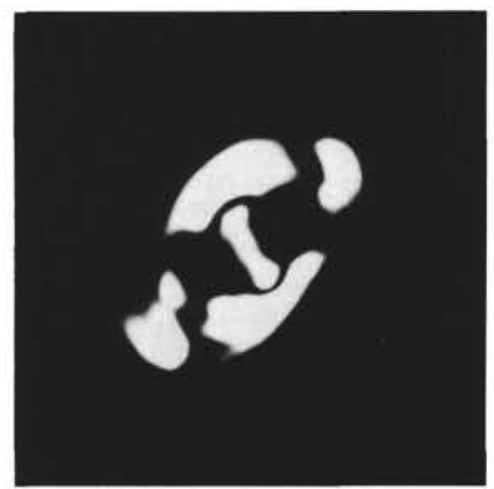

9

Plate 5. Light micrographs $(\times 3200)$ of lower Eocene nannofossils, New Jersey slope. (Crossed nicols unless otherwise indicated.) 1, 2. Helicosphaera seminulum Bramlette and Sullivan, Sample 612-58,CC (530.90 m), H. seminulum Subzone, (1) R95N25, (2) R102N21. 3. Helicosphaera seminulum Bramlette and Sullivan, Sample 612-57-6, 20-22 cm (528.71 m), H. seminulum Subzone, R102N8. 4, 5; 7-9. Helicosphaera seminulum Bramlette and Sullivan, Sample 612-55,CC (511.50 m), H. seminulum Subzone, (4) R92N14, (5) R103N23; (7) R91N24A, (8) R91N29A, partly crossed nicols, blue filter, same specimen as (7), (9) R104N3. 6. Helicosphaera seminulum Bramlette and Sullivan, Sample 612-56,CC $(521.00 \mathrm{~m})$, H. seminulum Subzone, R103N7. 


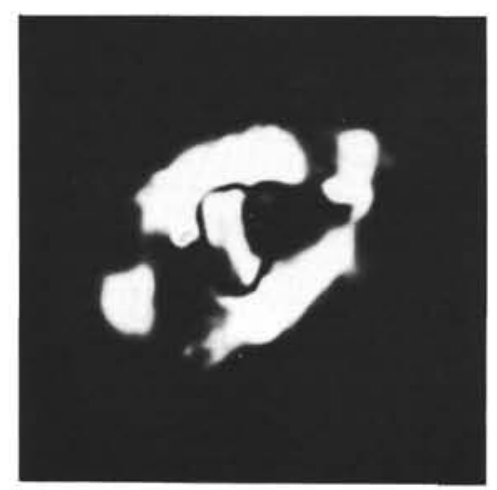

1

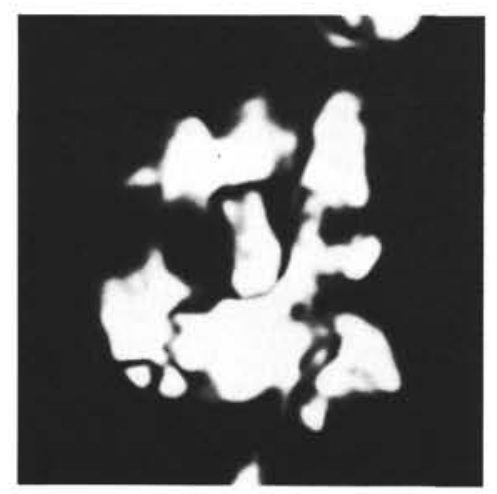

4

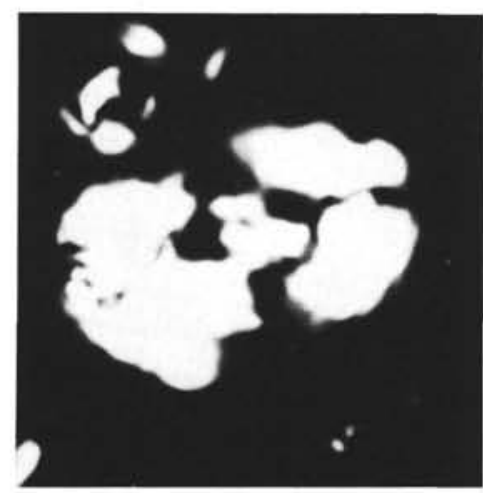

7

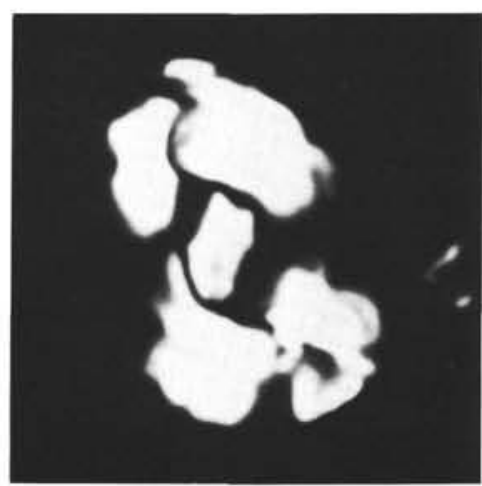

2

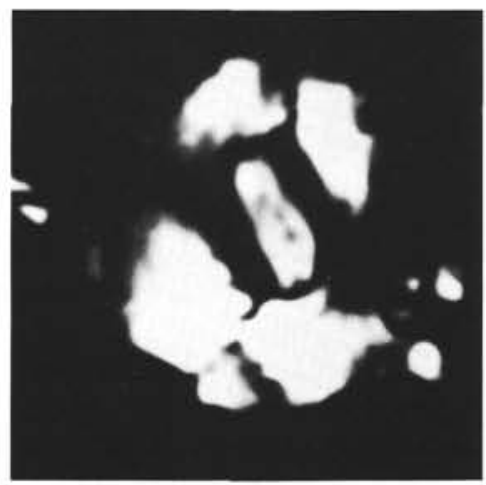

5

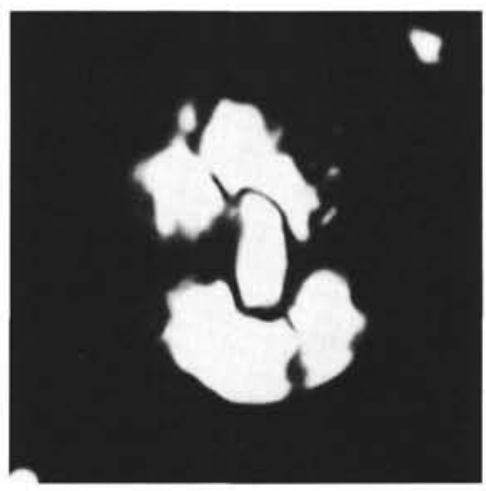

8

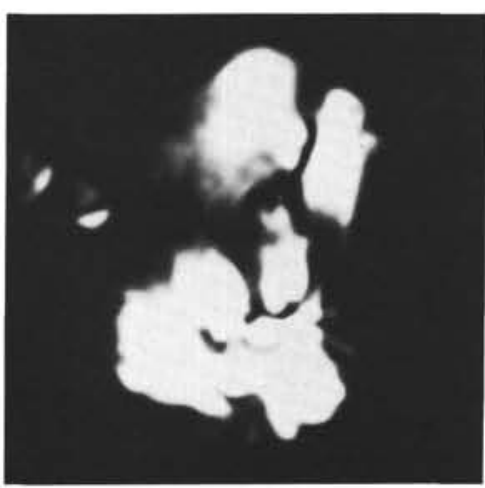

3

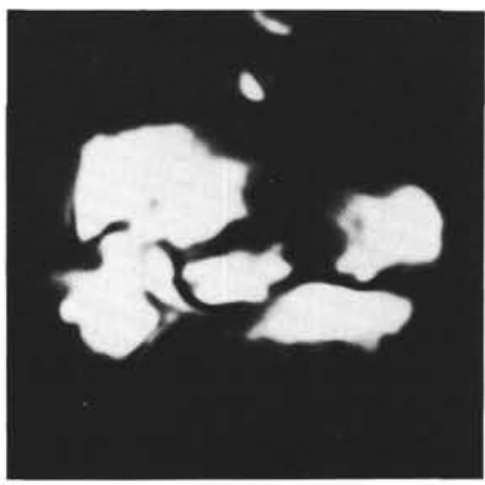

6

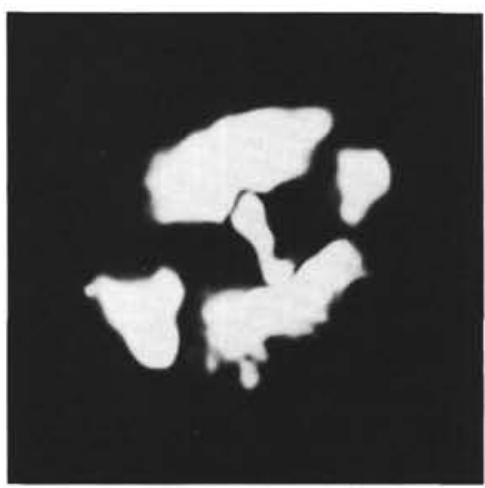

9

Plate 6. Light micrographs $(\times 3200)$ of lower Eocene nannofossils, New Jersey slope. (Crossed nicols.) 1, 9. Helicosphaera seminulum Bramlette and Sullivan, (1) Sample 612-54-5, 20-22 cm (498.71 m), H. lophota Subzone, R108N20, (9) Sample 612-43-1, 20-22 cm (386.21 m), D. kuepperi Subzone, R111N11. 2-6. Helicosphaera lophota (Bramlette and Sullivan) from H. lophota Subzone, (2) Sample 612-54-5, 20-22 cm (498.71 m), R108N18, (3) Sample 612-52-2, 20-22 cm (474.81 m), R107N19, (4) Sample 612-55-1, 20-22 cm (502.21 m), R108N24, (5) Sample 612-54-3, 20$22 \mathrm{~cm}(495.71 \mathrm{~cm}), \mathrm{R} 107 \mathrm{~N} 7,(6) \mathrm{R} 107 \mathrm{~N} 21$, same specimen as (3), rotated $90^{\circ}$. 7. Helicosphaera sp. cf. H. lophota (Bramlette and Sullivan), Sample 612-54,CC (499.93 m), H. lophota Subzone, R108N22. 8. Helicosphaera lophota (Bramlette and Sullivan), Sample 612-43-1, 20-22 cm $(386.21 \mathrm{~m})$, D. kuepperi Subzone, R111N25. 


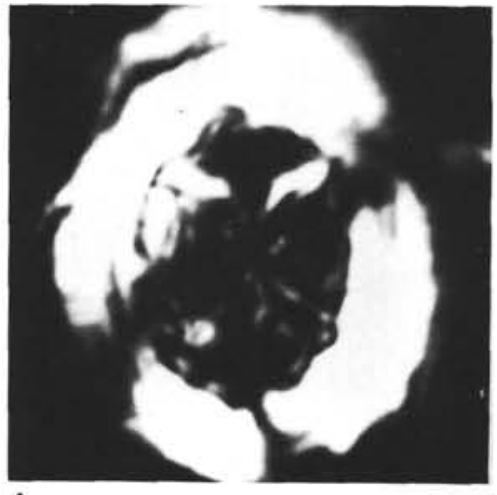

1

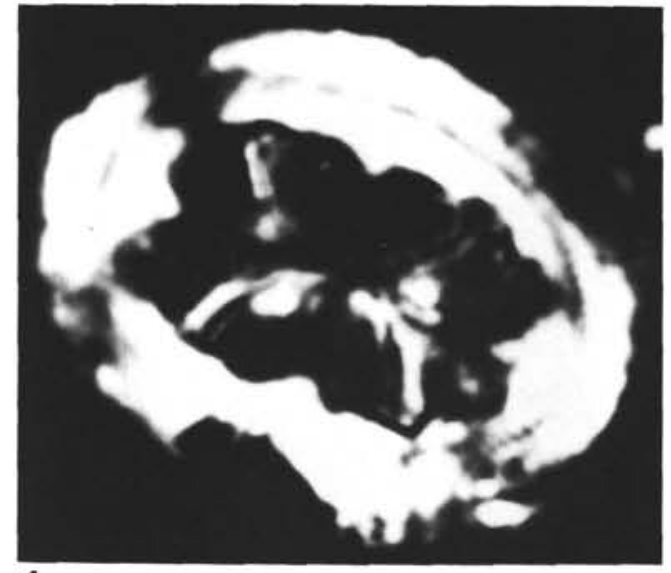

4

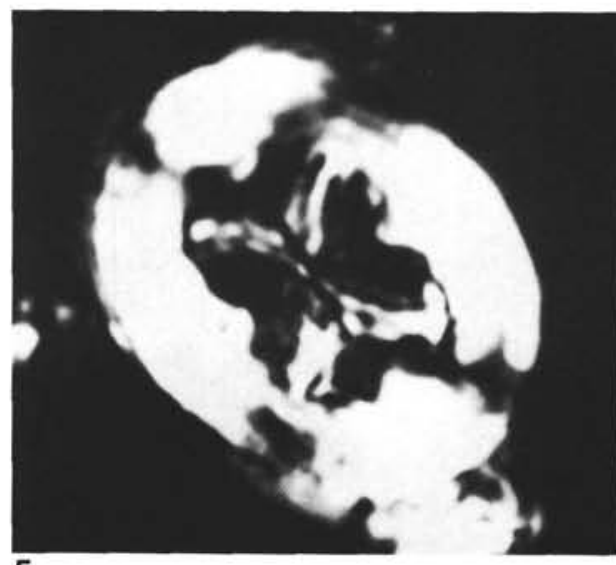

5

2
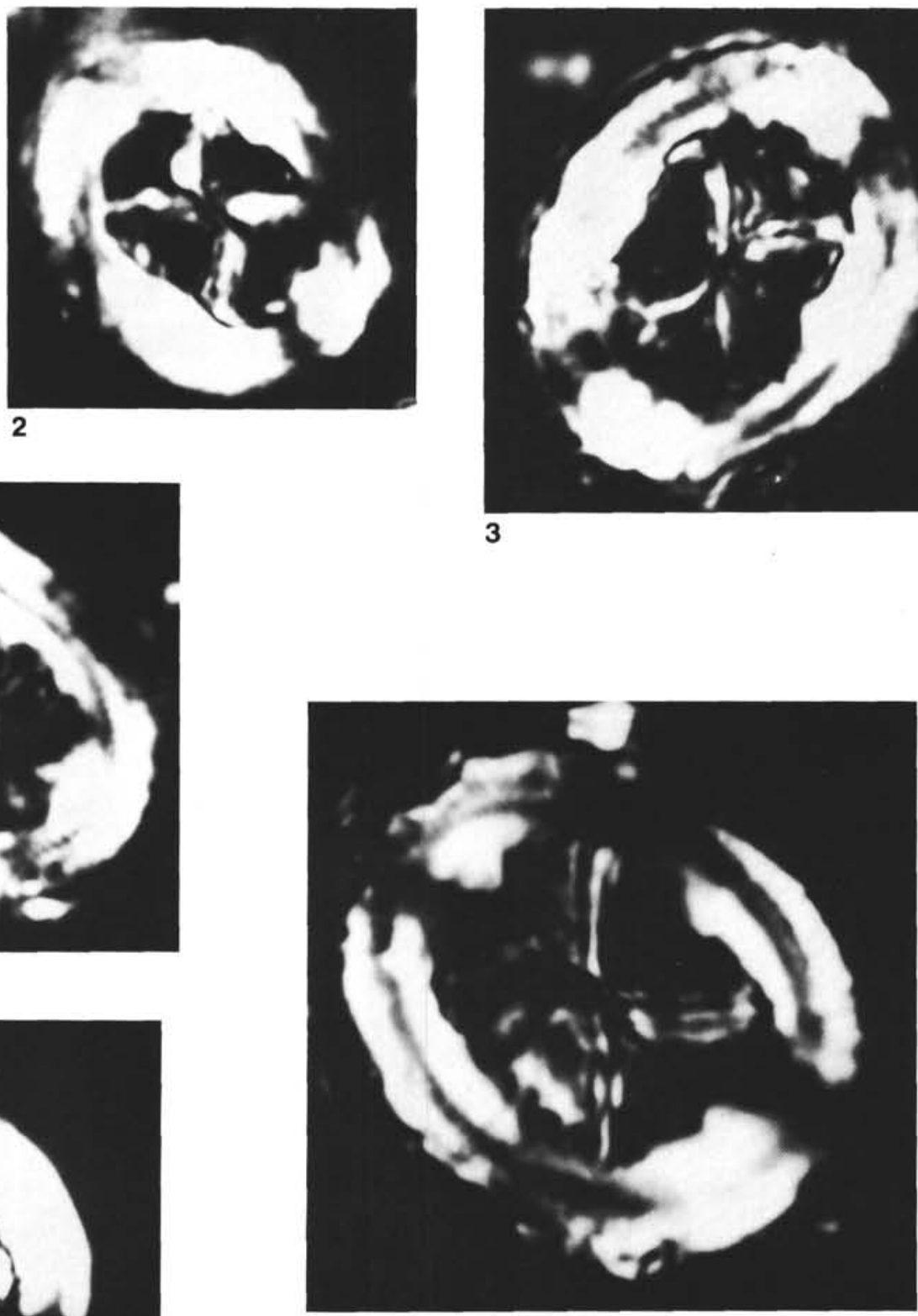

6

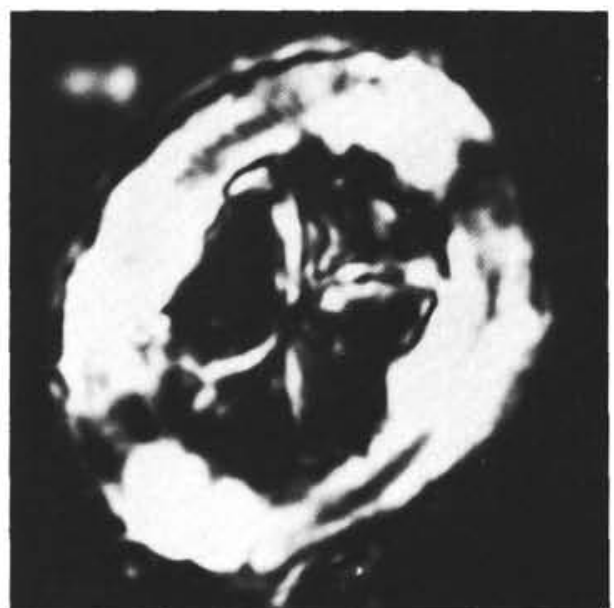

3

Plate 7. Light micrographs $(\times 3200)$ of lower Eocene (1-4) and middle Eocene $(5,6)$ nannofossils, New Jersey slope. (Crossed nicols.) 1, 2. Chiasmolithus grandis (Bramlette and Riedel), Sample 612-56,CC (521.00 m), H. seminulum Subzone, (1) R103N15, (2) R103N17, same specimen as

(1), rotated 45 ${ }^{\circ}$ 3-6. Chiasmolithus grandis (Bramlette and Riedel), (3) Sample 612-53,CC (483.43 m), H. lophota Subzone, R106N17, (4) Sample 612-43-1, 20-22 cm (386.21 m), D. kuepperi Subzone, R111N3, (5) Sample 612-37,CC (337.70 m), $R$. inflata Subzone, R104N29, (6) Sample 612-24,CC (212.35 m), middle Eocene, R93N13A. 


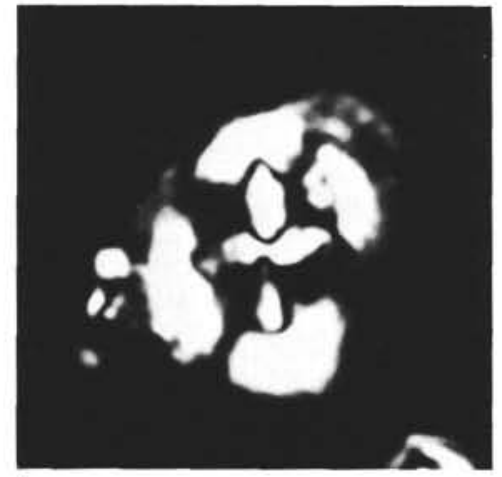

1

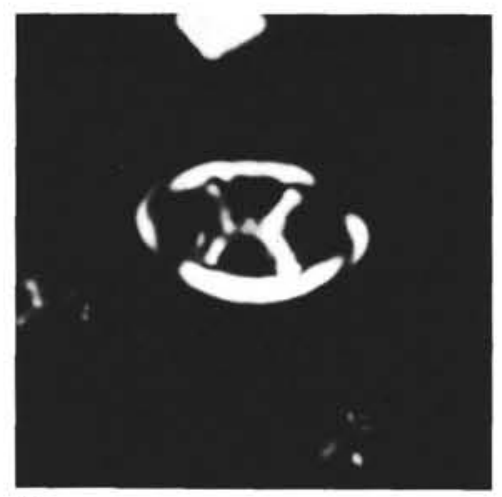

4

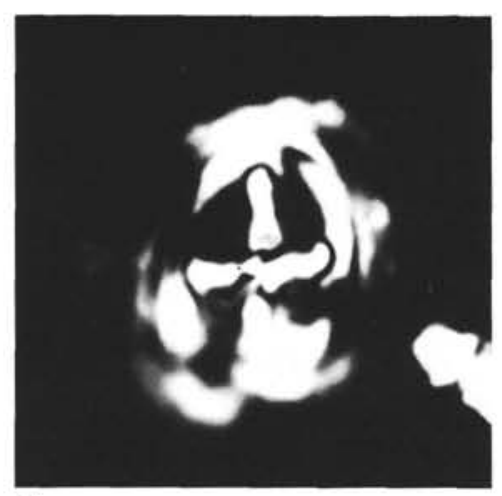

7

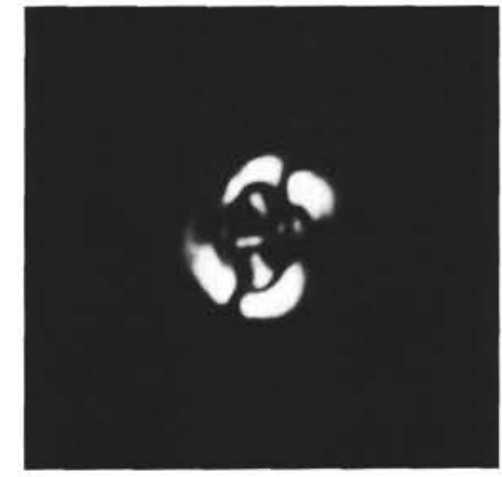

2

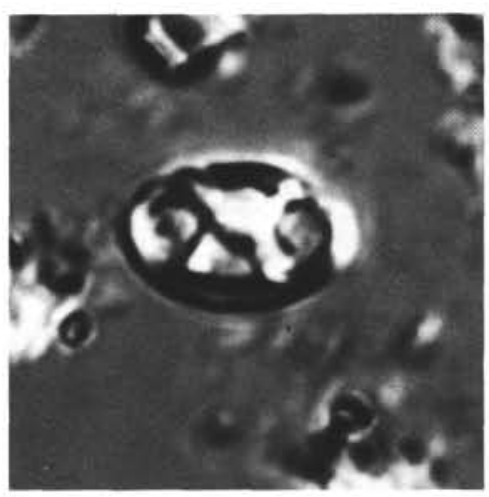

5

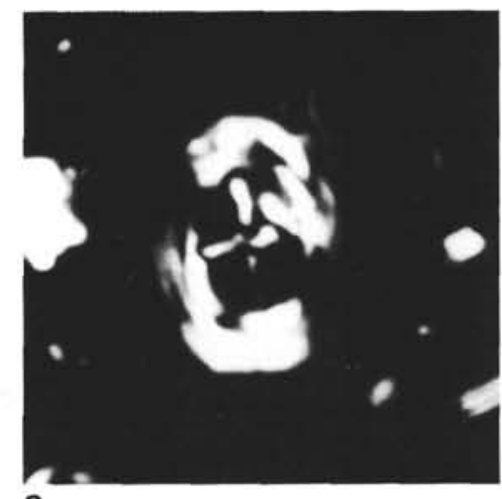

8

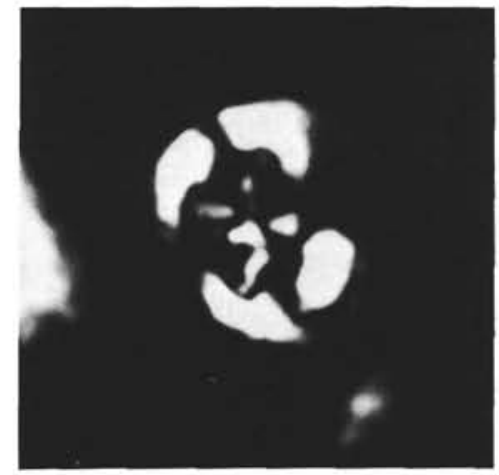

3

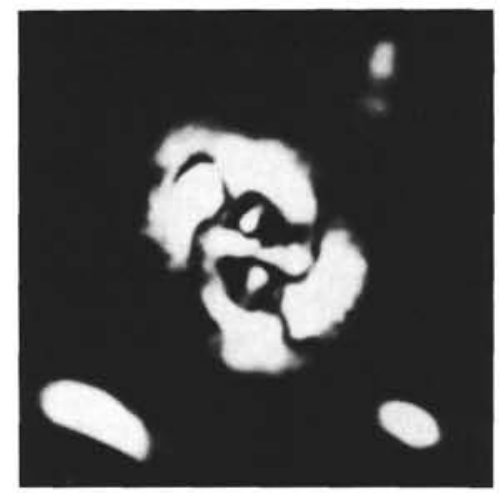

6

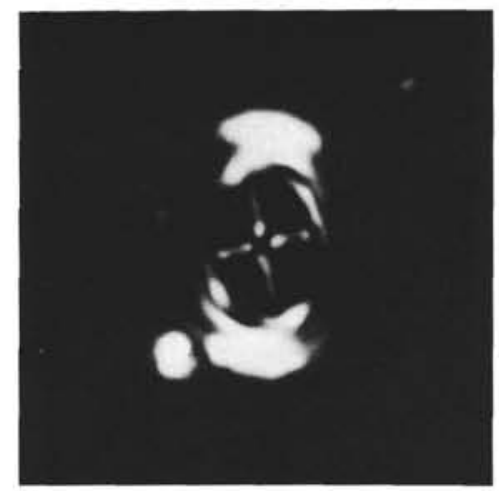

9

Plate 8. Light micrographs $(\times 3200)$ of lower Eocene $(1-5,7,8)$ and middle Eocene $(6,9)$ nannofossils, New Jersey slope. (Crossed nicols unless otherwise indicated.) 1. Chiasmolithus californicus (Sullivan), Sample 612-57,CC (530.40 m), H. seminulum Subzone, R102N26A. 2, 3, 6. Chiasmolithus consuetus (Bramlette and Sullivan), (2) Sample 612-55, CC (511.50 m), H. seminulum Subzone, R92N11, (3) Sample 612-57-5, 20$22 \mathrm{~cm}(527.21 \mathrm{~m}), H$. seminulum Subzone, R101N5; (6) Sample 612-35,CC (318.27 m), middle Eocene, R94N7. 4, 5. Neococcolithes dubius (Deflandre), Sample 612-51,CC (471.37 m), H. lophota Subzone, (4) R108N8, (5) R108N11, interference contrast, blue filter, same specimen as (4). 7-9. Campylosphaera dela (Bramlette and Sullivan), (7) Sample 612-55,CC (511.50 m), H. seminulum Subzone, R103N31, (8) Sample 61255,CC (511.50 m), H. seminulum Subzone, R91N18A, (9) Sample 612-24,CC (212.35 m), middle Eocene, R93N30A. 


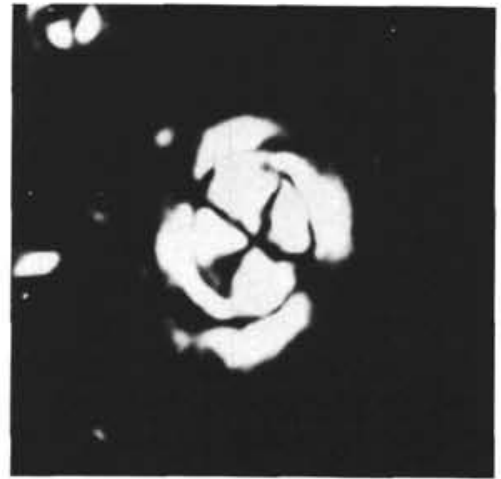

1

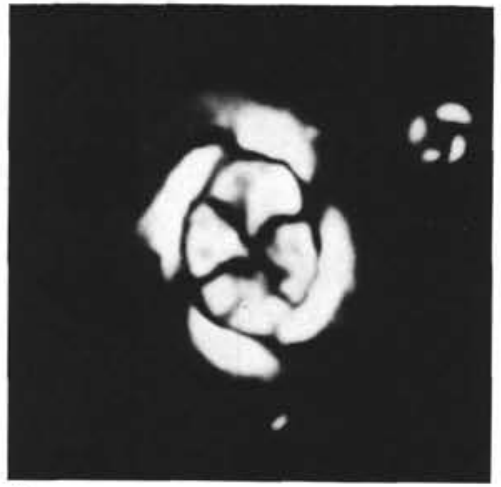

4

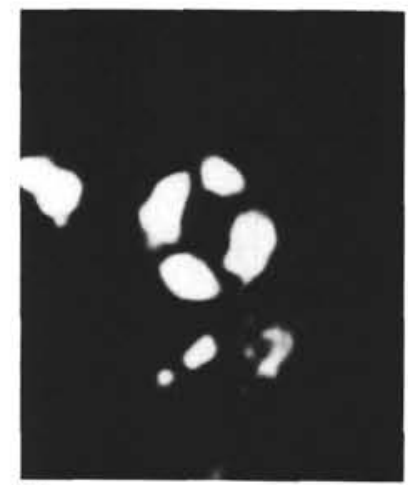

7

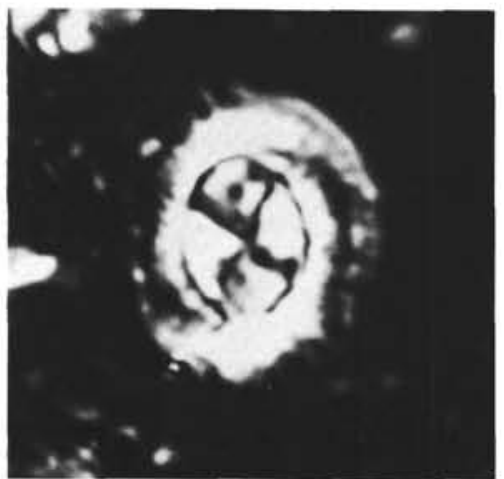

2

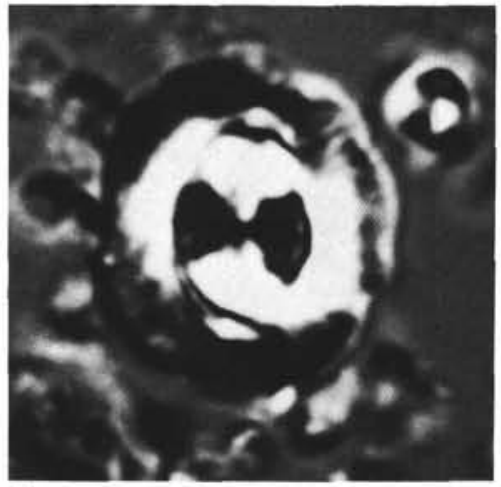

5

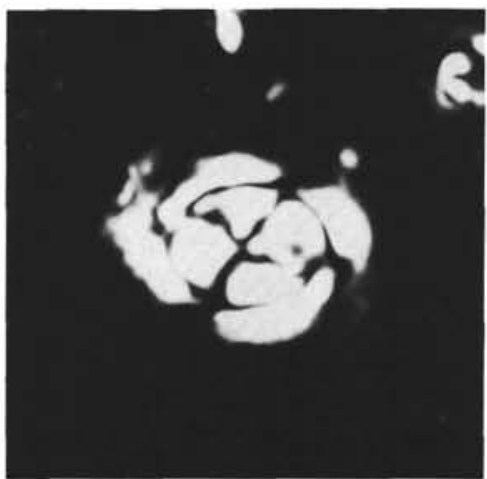

3

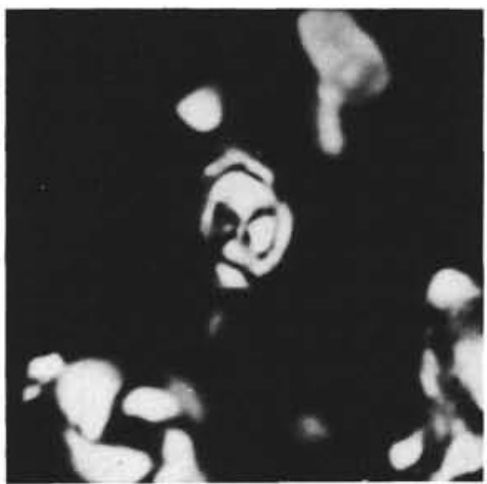

6

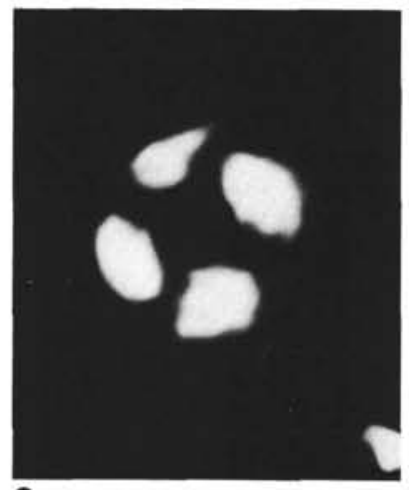

8

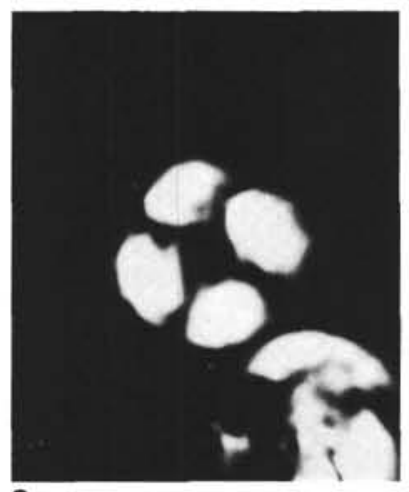

9

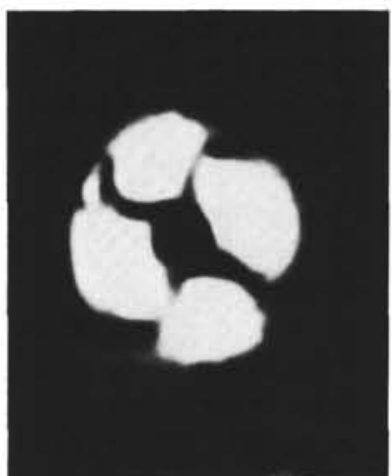

10

Plate 9. Light micrographs $(\times 3200)$ of lower Eocene $(1-5,7-10)$ and middle Eocene (6) nannofossils, New Jersey slope. (Crossed nicols except where otherwise indicated.) 1-3. Coccolithus cribellum (Bramlette and Sullivan), Sample 612-54-3, 20-22 cm (495.71 m), H. lophota Subzone, all same specimen, (1) R105N27, (2) R105N31, interference contrast, blue filter, (3) R105N29, rotated 90 . 4, 5. Coccolithus cribellum (Bramlette and Sullivan), Sample 612-51,CC (471.37 m), H. lophota Subzone, (4) R92N30, (5) R92N34, interference contrast, same specimen as (4).6. Coccolithus cribellum (Bramlette and Sullivan), Sample 612-24,CC (212.35 m), middle Eocene, R93N25A. 7-10. Cyclococcolithina formosa (Kamptner), (7) Sample 612-60,CC (550.59 m), D. binodosus Subzone, R97N21, (8) Sample 612-55,CC (511.50 m), H. seminulum Subzone, R92N3, (9) Sample 612-47,CC (434.24 m), C. pseudogammation Subzone, R115N19, (10) Sample 612-43-1, 20-22 cm (386.21 m), D. kuepperi Subzone, R110N10A. 


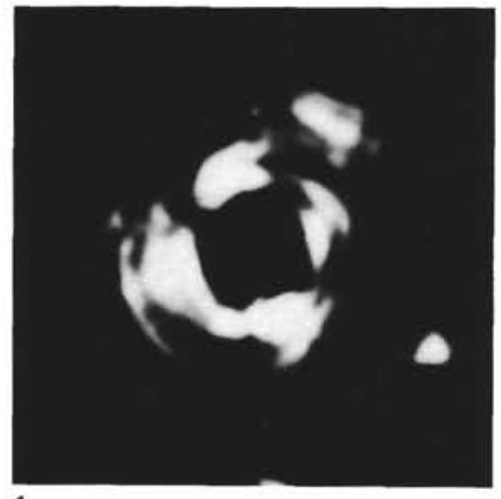

1

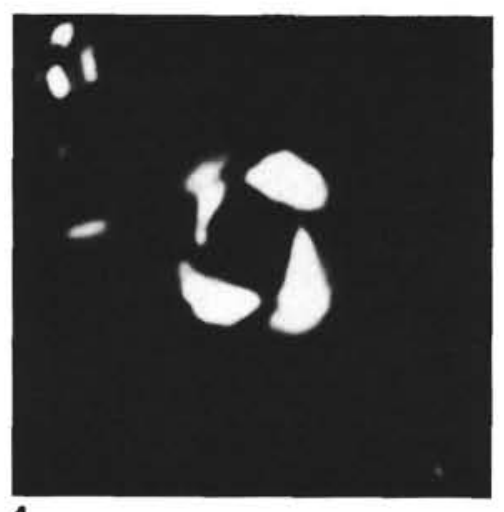

4

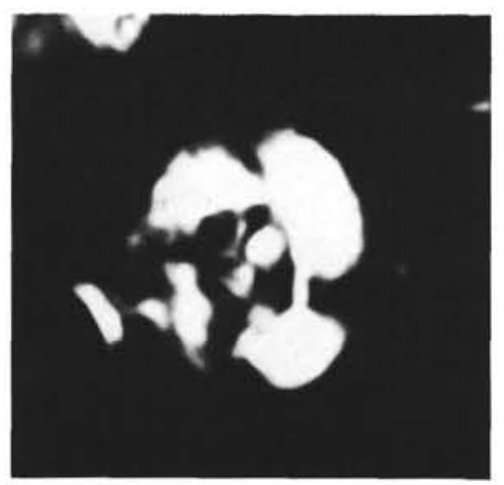

7

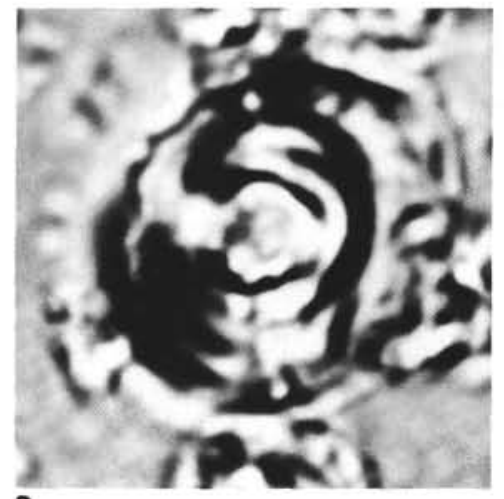

2

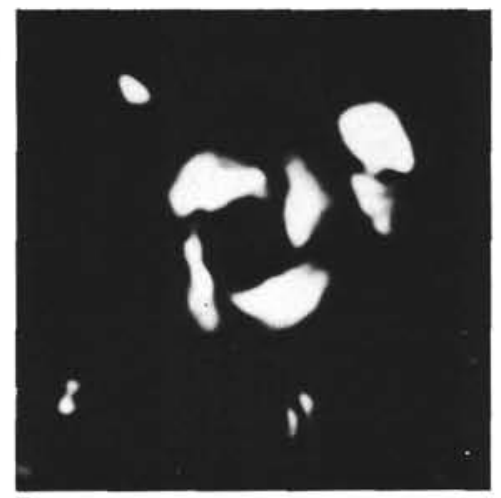

5

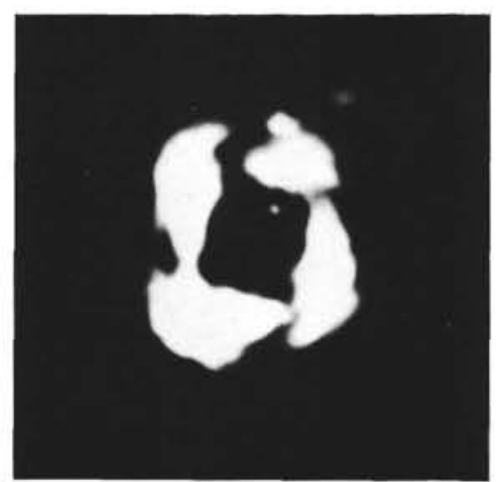

8

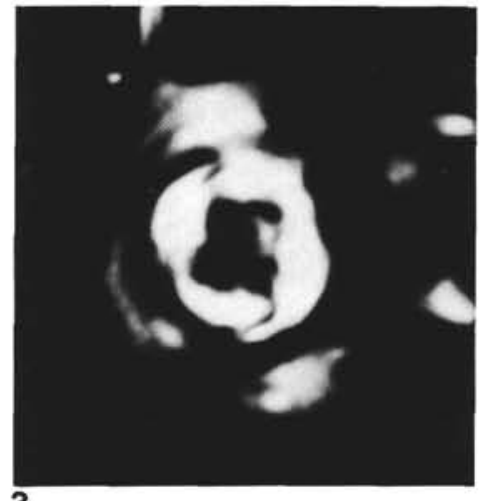

3

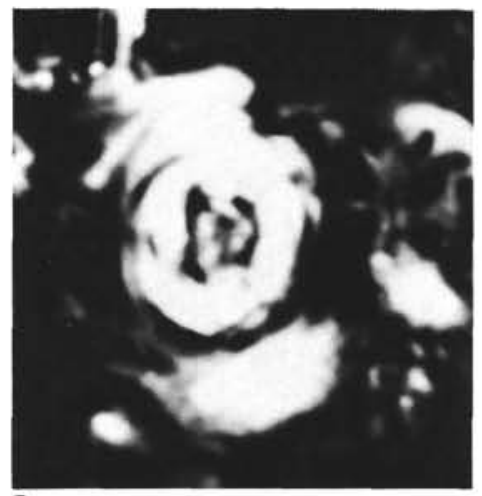

6

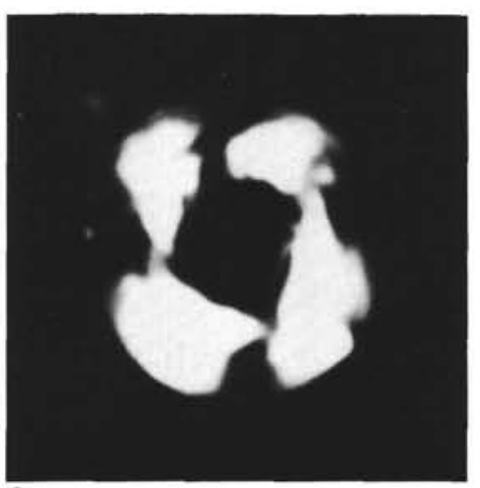

9

Plate 10. Light micrographs $(\times 3200)$ of lower Eocene nannofossils, New Jersey slope. (Crossed nicols except where otherwise indicated.) $1,2$. Coccolithus magnicrassus Bukry, Sample 612-54,CC (499.93 m), H. lophota Subzone, (1) R90N6A, (2) R90N12A, partly crossed nicols, blue filter, same specimen as (1). 3, 6. Coccolithus magnicrassus Bukry, Sample 612-51,CC (471.37 m), H. lophota Subzone, (3) R93N7A, (6) R93N11A, interference contrast, same specimen at (3). 4, 5; 7-9. Reticulofenestra spp., (4) Sample 612-55-6, 20-22 cm (509.71 m), H. seminulum Subzone, R108N30, (5) Sample 612-55-3, 20-22 cm (505.21 m), H. seminulum Subzone, R108N28, (7) Sample 612-52-2, 20-22 cm (474.81 m), H. lophota Subzone, R107N23, (8) Sample 612-51,CC (471.37 m), H. lophota Subzone, R108N4, (9) Sample 612-43-1, 20-22 cm (386.21 m), D. kuepperi Subzone, R111N13. 


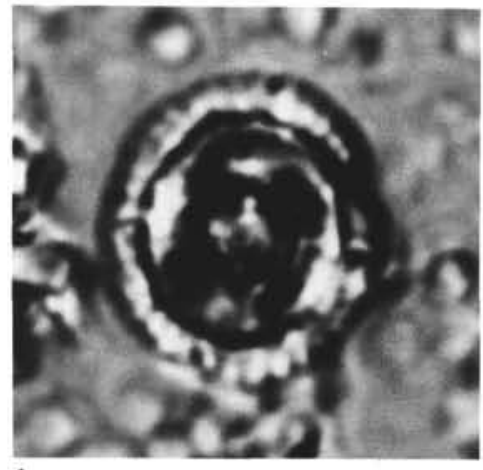

1

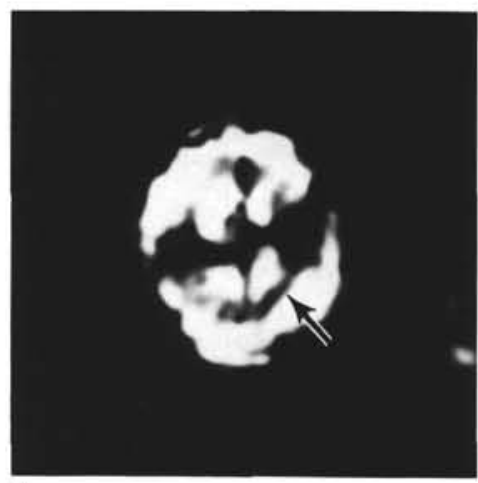

4

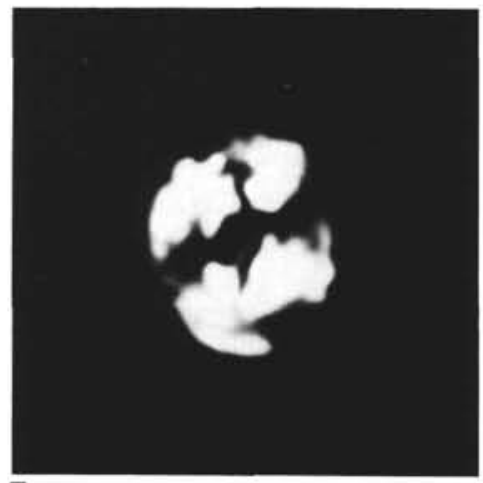

7

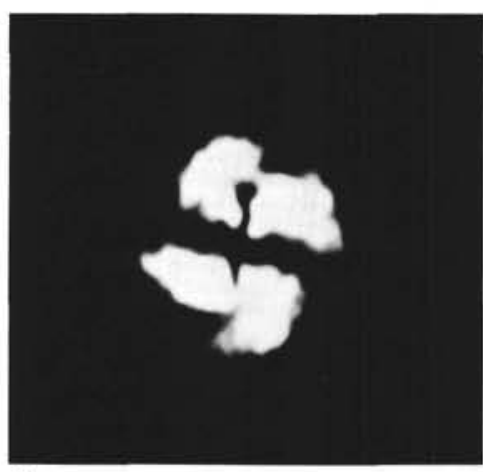

2

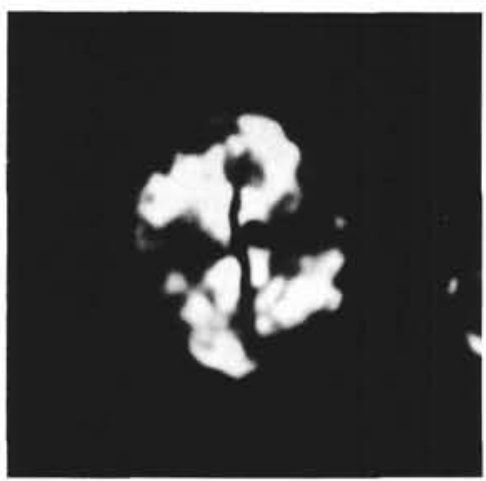

5

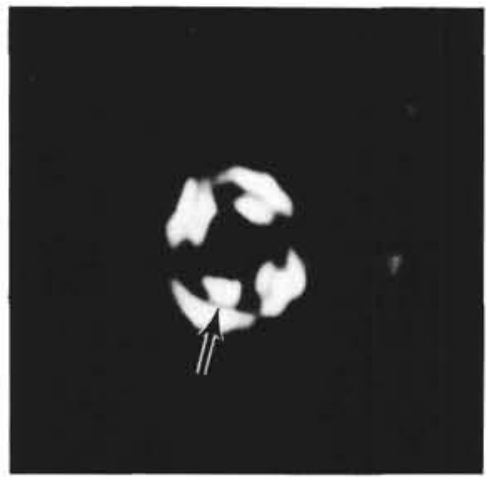

8

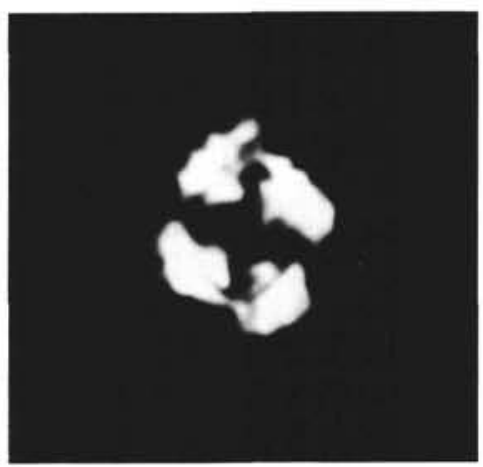

3

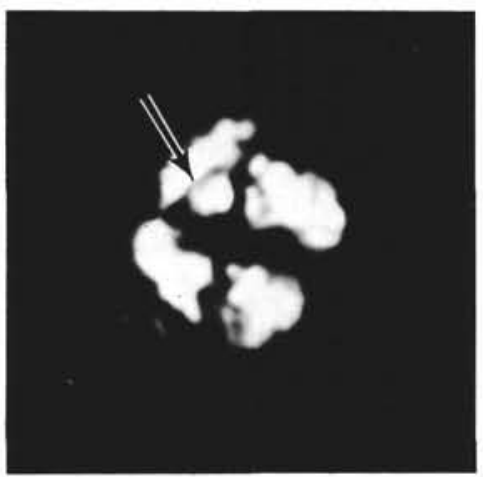

6

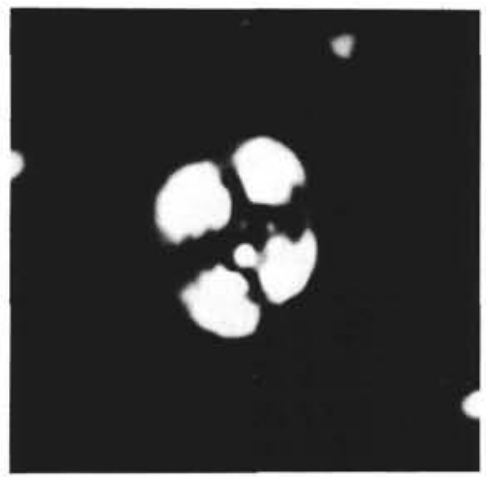

9

Plate 11. Light micrographs $(\times 3200)$ of lower Eocene nannofossils, New Jersey slope. (Crossed nicols unless otherwise indicated.) 1, 2. Coccolithus crassus Bramlette and Sullivan, Sample 612-47,CC (434.24 m), C. pseudogammation Subzone, (1) R114N20, partly crossed nicols, blue filter, (2) R114N19, same specimen as (1). 3-6. Coccolithus crassus Bramlette and Sullivan, Sample 612-43-1, 20-22 cm (386.21 m), D. kuepperi Subzone, (3) R115N7, (4) R114N31, focus on central tube, (5) R114N36, focus on extinction figure, same specimen as (4), (6) R115N9. 7-9. Coccolithus crassus Bramlette and Sullivan, (7) Sample 612-48-5, 20-22 cm (440.51 m), C. pseudogammation Subzone, R114N23, (8) Sample 612-54-1, 20-22 cm (492.71 m), H. lophota Subzone, R115N4, (9) Sample 612-54,CC (499.93 m), H. lophota Subzone, R112N3 (note: arrow points to characteristic orange band on bright shield; not always visible on prints). 


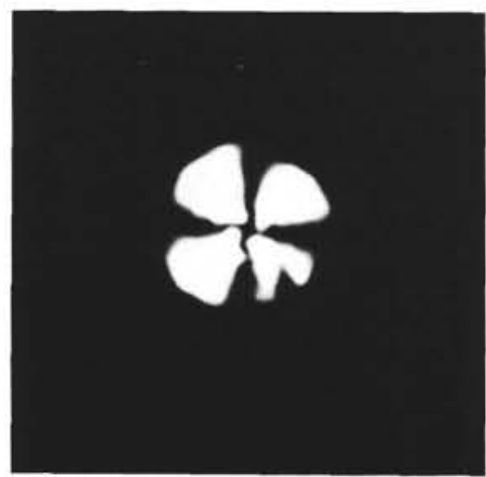

1

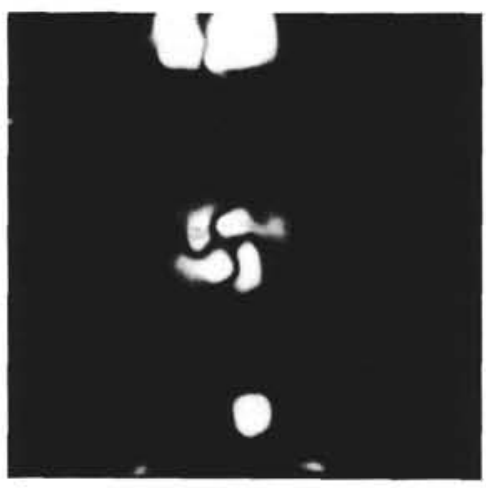

4

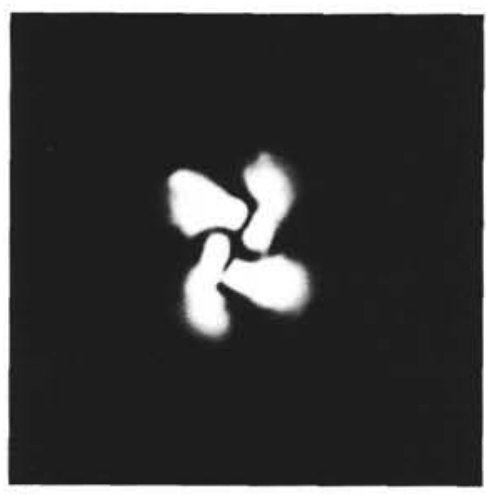

7

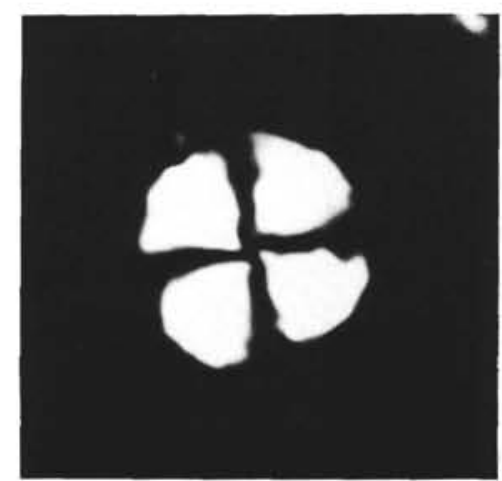

2

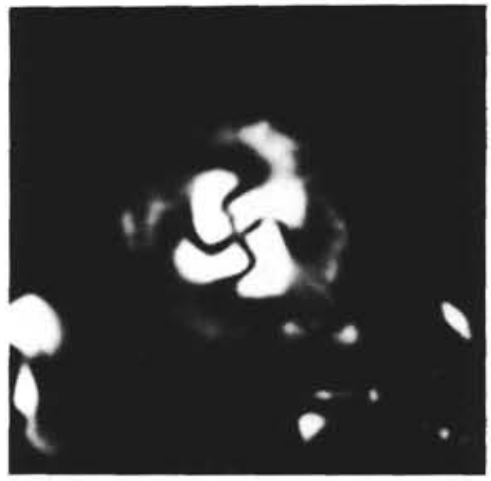

5

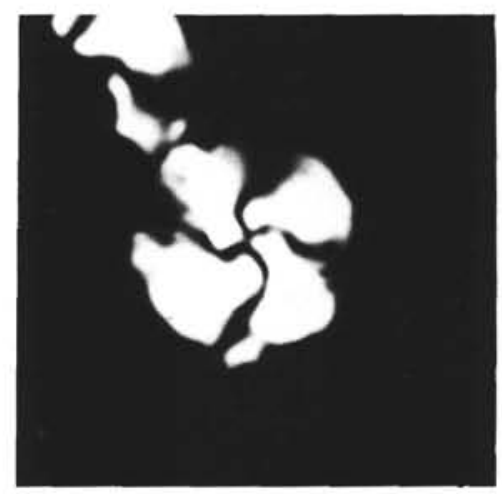

8

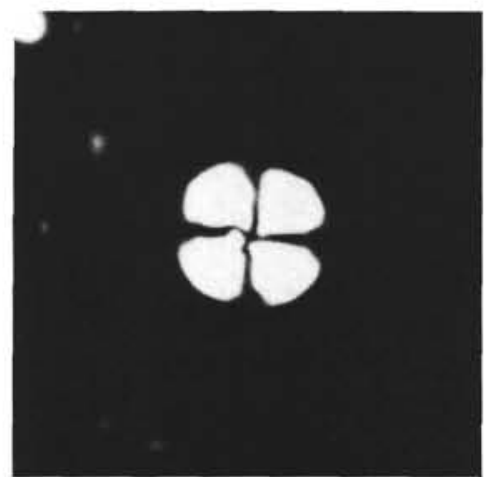

3

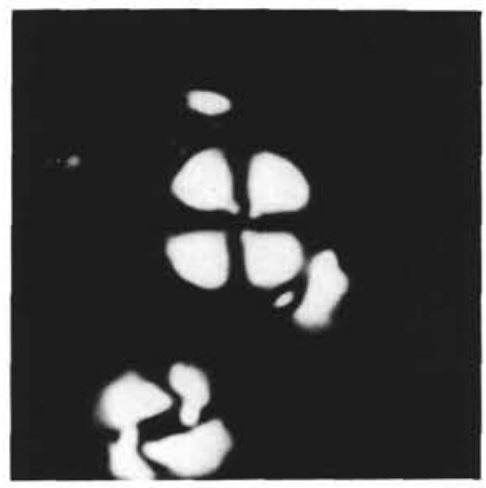

6

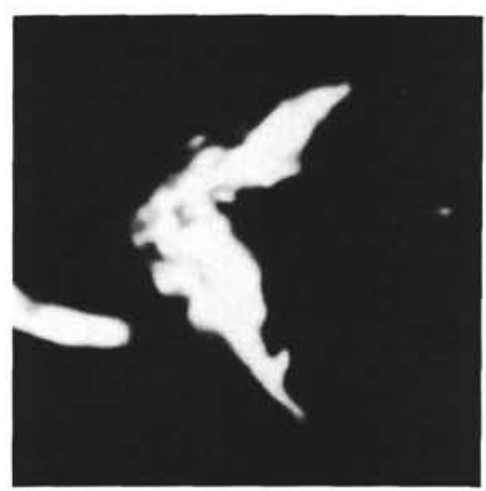

9

Plate 12. Light micrographs $(\times 3200)$ of lower Eocene nannofossils $(1-5,7-8)$ and middle Eocene $(6,9)$, New Jersey slope. (Crossed nicols.) 1-3, 6. Cepekiella lumina (Sullivan), (1) Sample 612-57-6, 20-22 cm (528.71 m), H. seminulum Subzone, R102N15, (2) Sample 612-53,CC (483.43 m), H. lophota Subzone, R107N13, (3) Sample 612-43-1, 20-22 cm (386.21 m), D. kuepperi Subzone, R111N8, (6) Sample 612-24,CC (212.35 m), middle Eocene, R93N22A. 4, 5. Cyclococcolithina gammation (Bramlette and Sullivan), (4) Sample 612-55,CC (511.50 m), H. seminulum Subzone, R92N9, (5) Sample 612-43-1, 20-22 cm (386.21 m), D. kuepperi Subzone, R111N27. 7, 8. Cyclicargolithus pseudogammation (Bouche), (7) Sample 612-43-1, 20-22 cm (386.21 m), D. kuepperi Subzone, R110N35A, (8) Sample 613-43-1, 20-21 cm (487.30 m), $R$. tenuis Subzone, R109N10A. 9. ?Nannotetrina fulgens (Stradner), Sample 612-37-3, $65 \mathrm{~cm}(331.75 \mathrm{~m}), R$. inflata Subzone, R114N5, broken specimen, 2 arms only. 


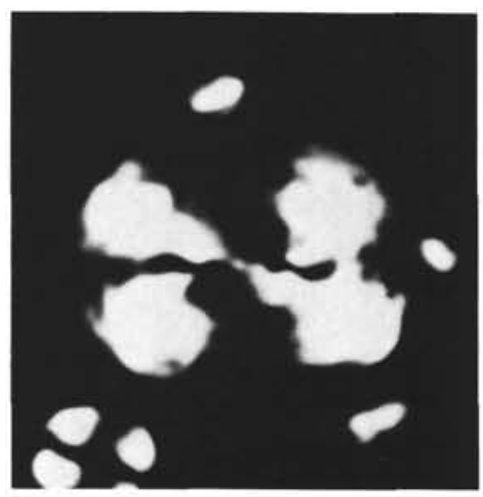

1

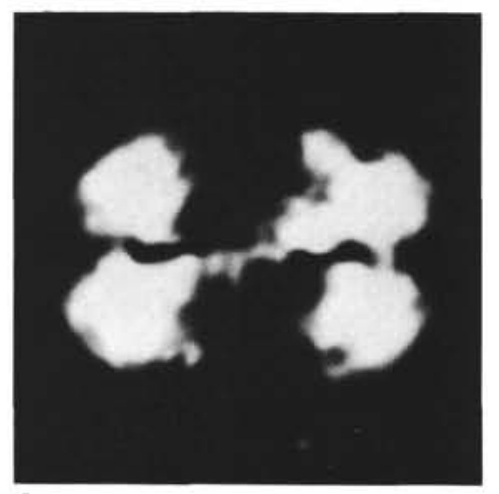

4

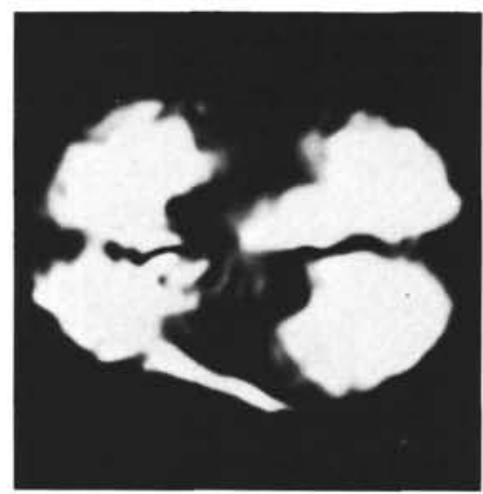

7

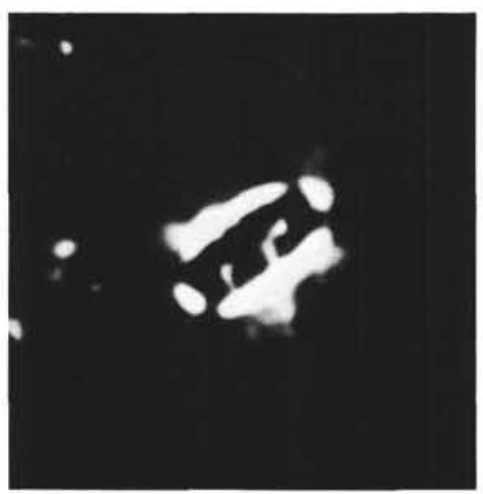

2

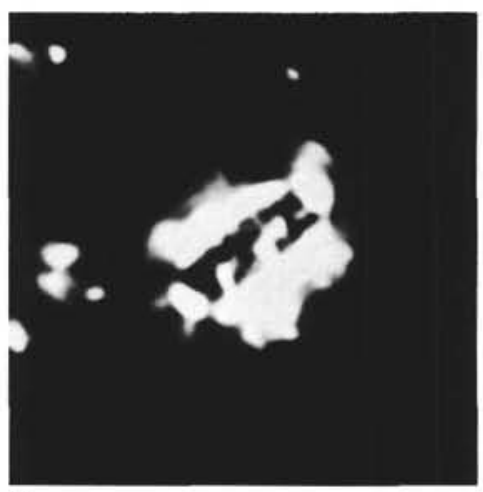

5

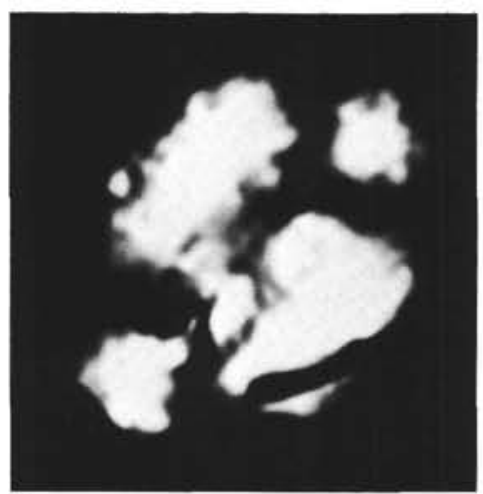

8

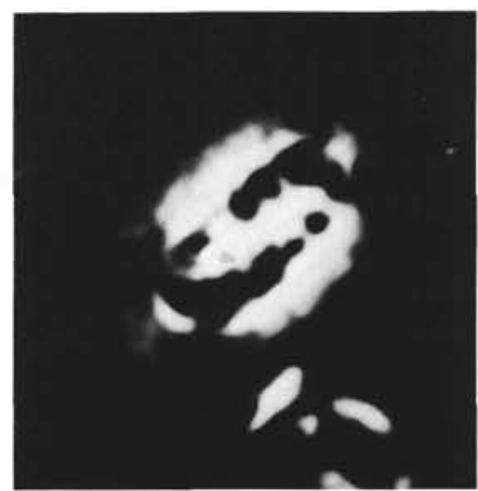

3

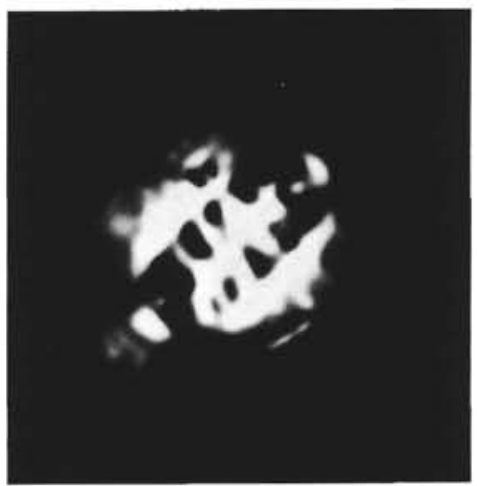

6

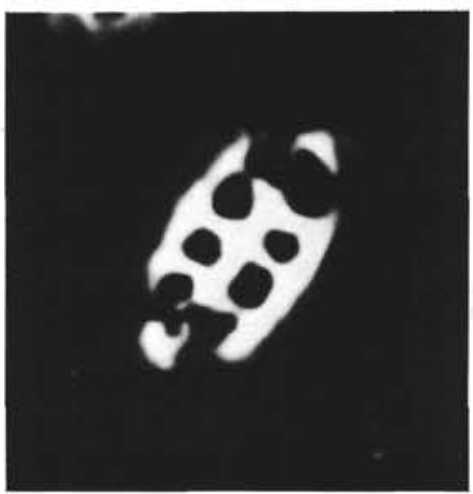

9

Plate 13. Light micrographs $(\times 3200)$ of lower Eocene nannofossils, New Jersey slope. (Crossed nicols.) 1, 4, 7, 8. Ellipsolithus macellus (Bramlette and Sullivan), (1) Sample 612-60,CC (550.59 m), D. binodosus Subzone, R105N33, (4) Sample 612-59,CC (549.80 m), D. binodosus Subzone, R96N21, (7) Sample 612-59-6, 20-22 cm (547.91 m), L. nascens Subzone, R116N14A, (8) R116N13A, same specimen as (7). 2, 5. Ellipsolithus lajollaensis Bukry and Percival, Sample 612-43-2, 20-22 cm (387.71 m), D. kuepperi Subzone, (2) R111N35, (5) R111N34, same specimen as (2). 3, 6, 9. Ellipsolithus distichus (Bramlette and Sullivan), (3) Sample 612-55,CC (511.50 m), H. seminulum Subzone, R90N16A, (6) Sample $612-56-3,20-22 \mathrm{~cm}(514.71 \mathrm{~m}), H$. seminulum Subzone, R115N33, (9) Sample 612-56-6, 20-22 cm (519.21 m), H. seminulum Subzone, R115N28. 


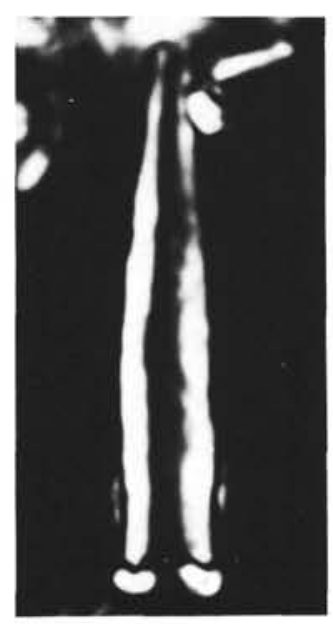

1

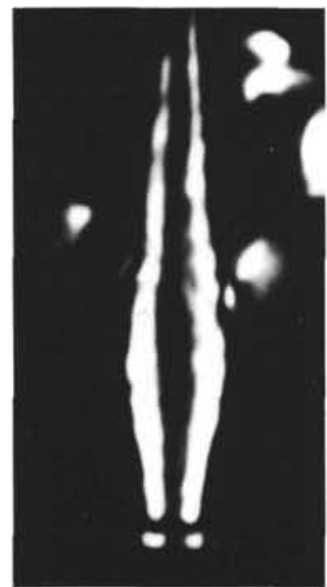

5

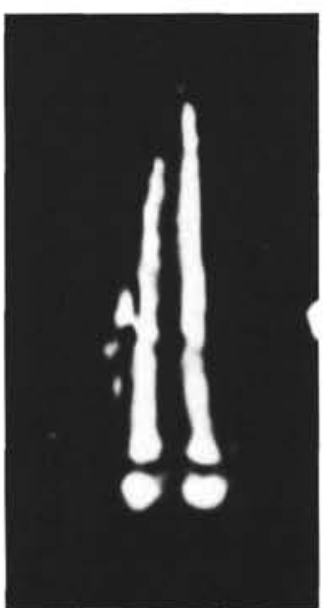

2

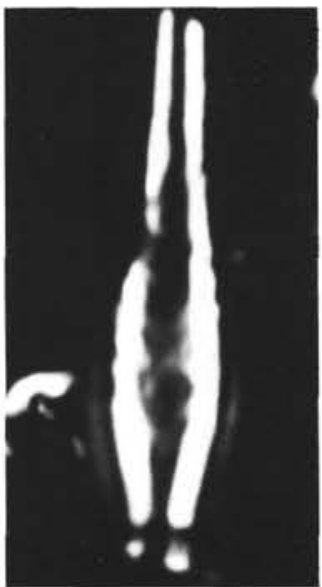

6

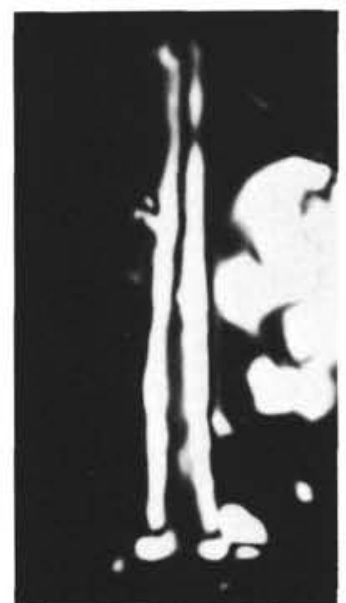

3

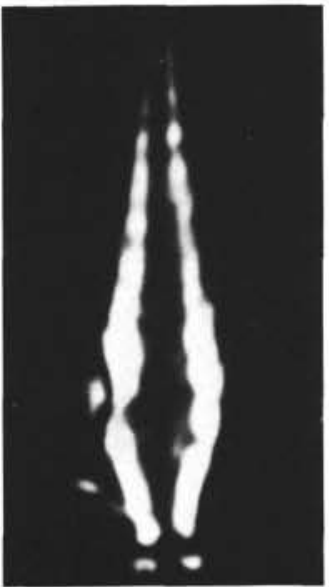

7

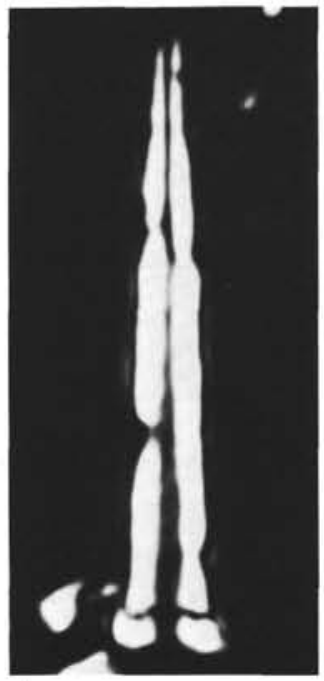

4

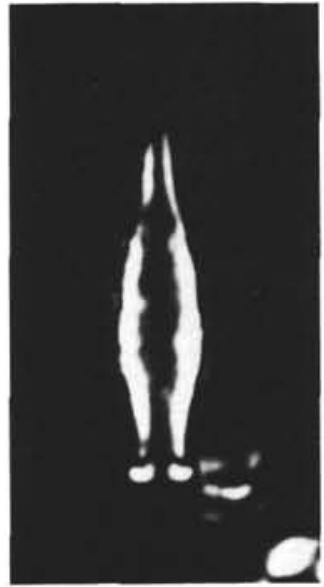

8

Plate 14. Light micrographs $(\times 3200)$ of lower Eocene $(1,2)$ and middle Eocene $(3-8)$ nannofossils, New Jersey slope. (Crossed nicols.) $1,2$. Rhabdosphaera tenuis Bramlette and Sullivan, (1) Sample 612-45,CC (414.32 m), R. tenuis Subzone, R112N18, (2) Sample 612-43-2, 20-22 cm $(387.71 \mathrm{~m})$, D. kuepperi Subzone, R111N33. 3, 4. Rhabdosphaera tenuis Bramlette and Sullivan, Sample $612-37-4,20-22 \mathrm{~cm}(332.81 \mathrm{~m}), R$. inflata Subzone, (3) R113N8, (4) R113N12. 5. Rhabdosphaera inflata Bramlette and Sullivan, Sample 612-37-6, 20-22 cm (335.81 m), . inflata Subzone, R114N7. 6-8. Rhabdosphaera inflata Bramlette and Sullivan, Sample 612-37-4, 20-22 cm (332.81 m), R. inflata Subzone, (6) R113N5, (7) R113N7, (8) R113N11. 